Copyright (c) 2010, American Society for Microbiology. All Rights Reserved.

\title{
Melanized Fungi in Human Disease
}

\author{
Sanjay G. Revankar ${ }^{1 *}$ and Deanna A. Sutton ${ }^{2}$ \\ Department of Medicine, Division of Infectious Diseases, Wayne State University, Detroit, Michigan, ${ }^{1}$ and \\ Fungus Testing Laboratory, University of Texas Health Science Center, San Antonio, Texas ${ }^{2}$
}

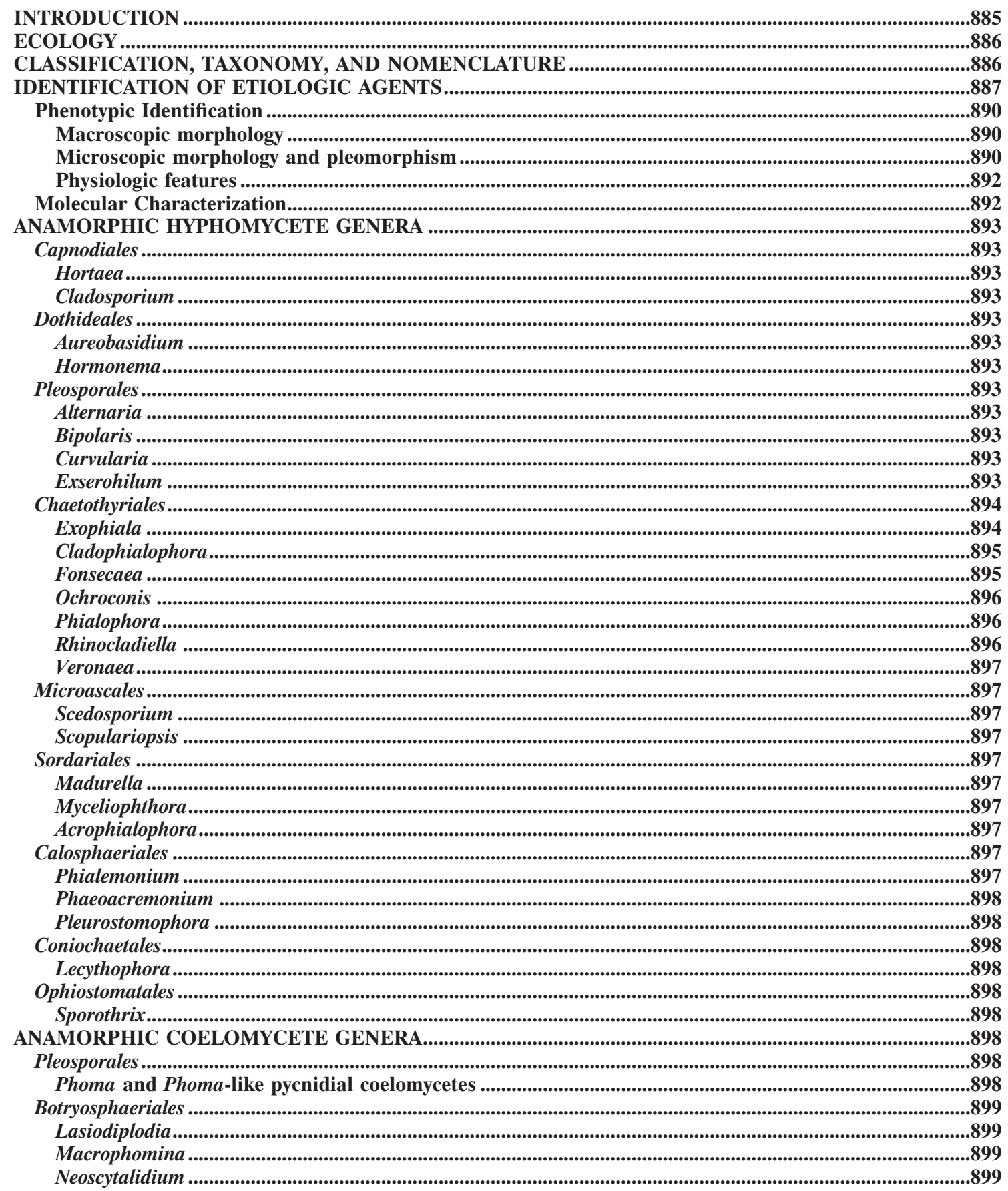

\footnotetext{
* Corresponding author. Mailing address: Harper University Hospital, 3990 John R. St., 5 Hudson, Detroit, MI 48201. Phone: (313) 745-8599. Fax: (313) 993-0302. E-mail: srevankar@med.wayne.edu.
} 


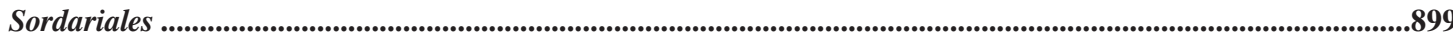

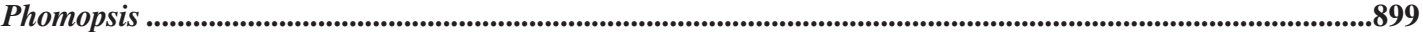

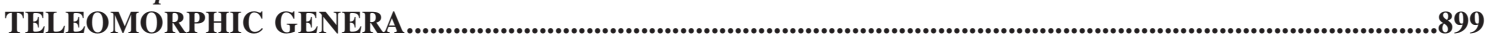

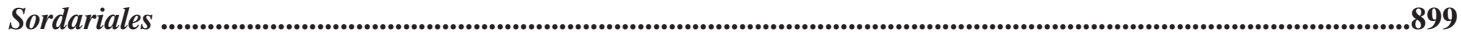

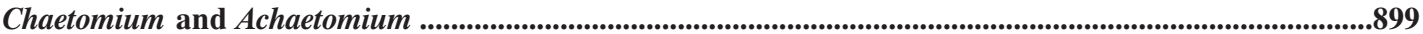

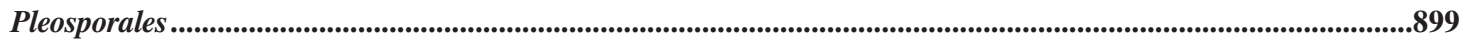

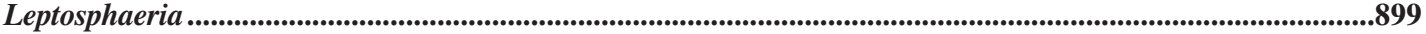

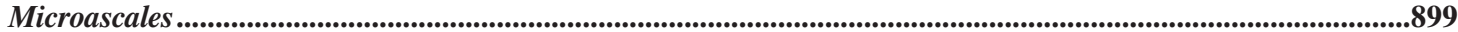

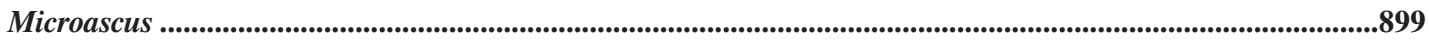

Pseudallescheria .......................................................................................................................................................900

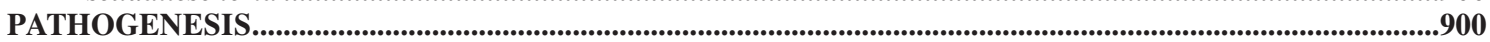

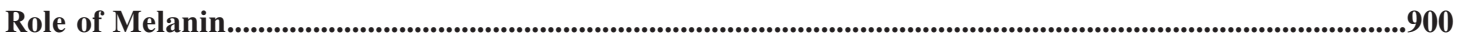

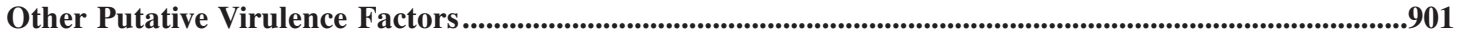

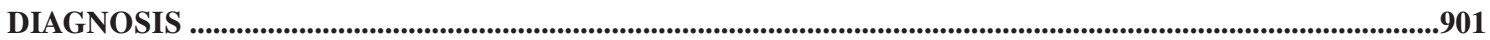

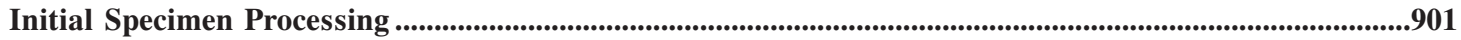

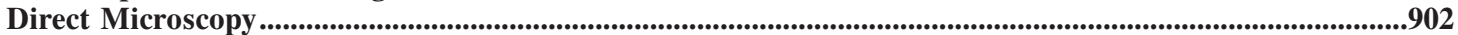

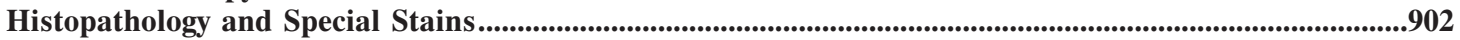

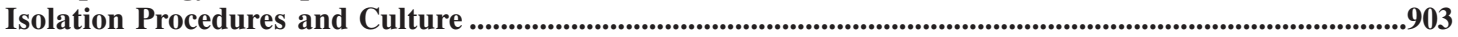

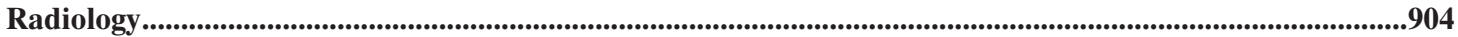

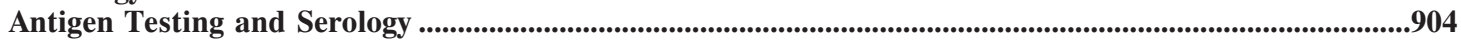

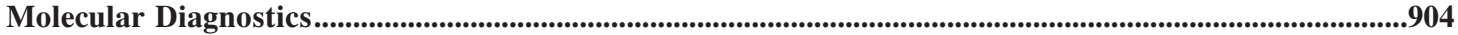

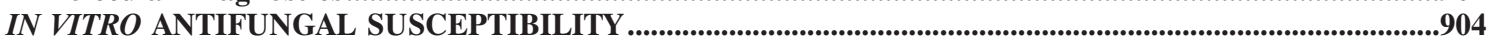

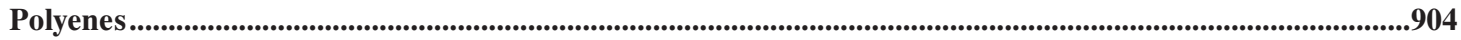

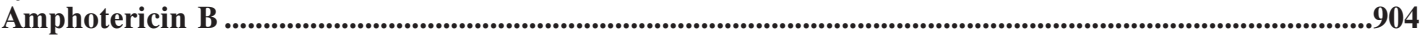

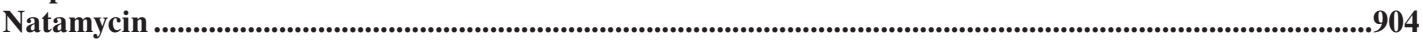

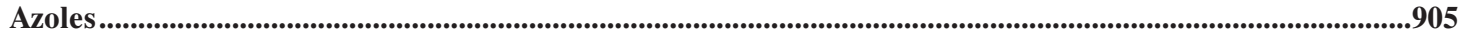

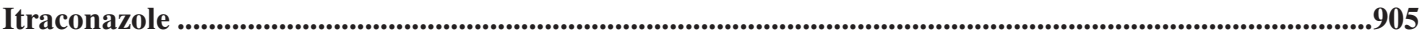

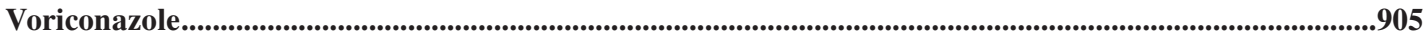

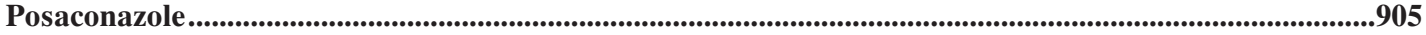

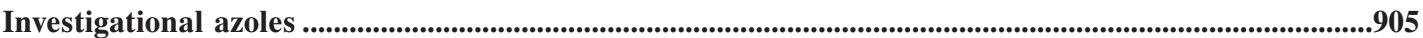

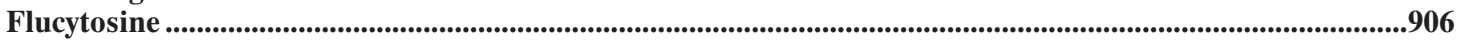

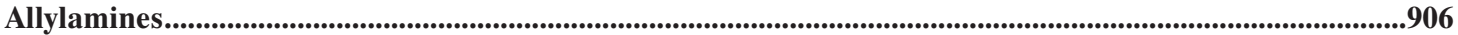

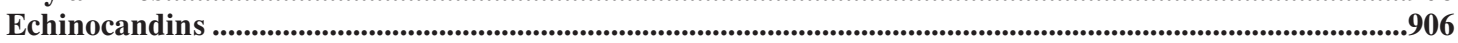

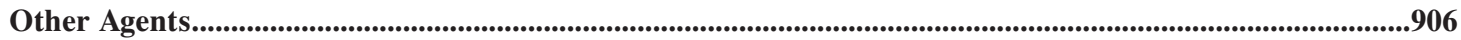

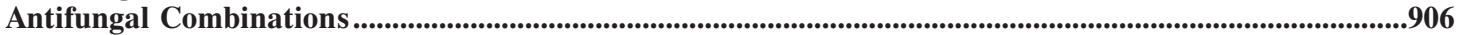

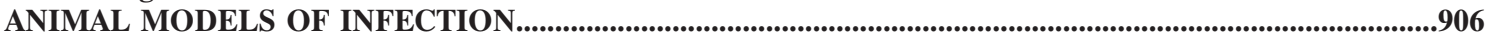

CLINICAL SYNDROMES AND THEIR MANAGEMENT .................................................................................907

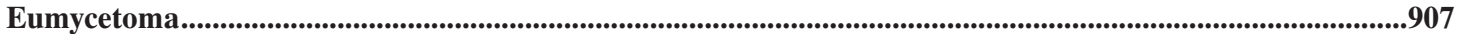

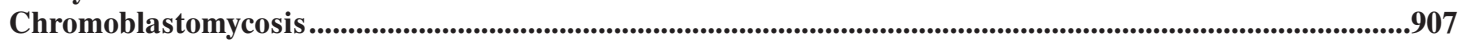

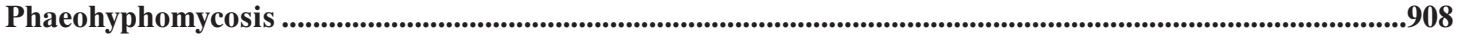

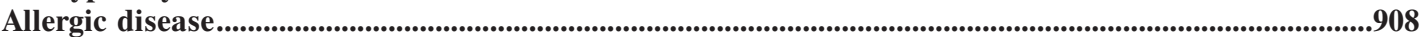

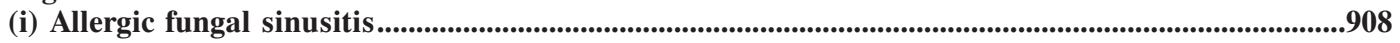

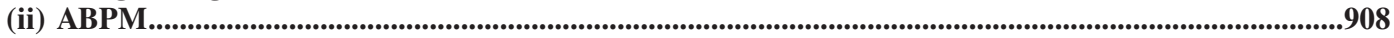

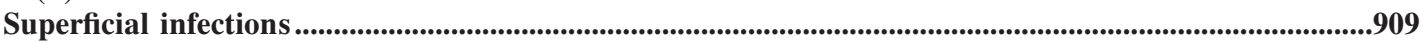

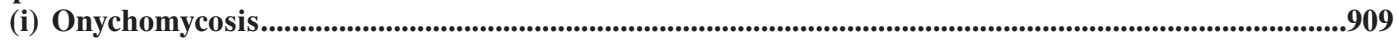

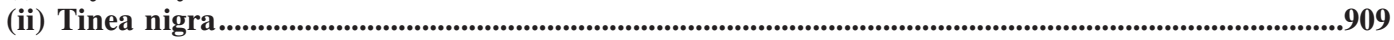

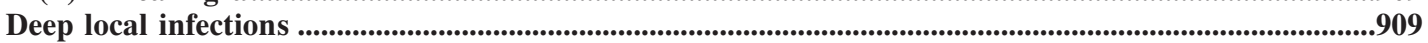

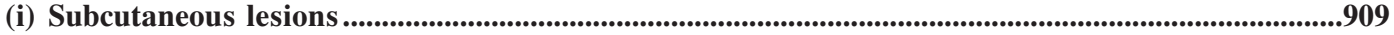

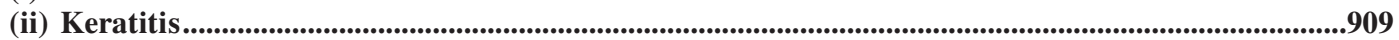

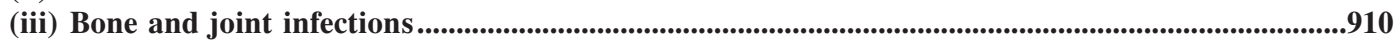

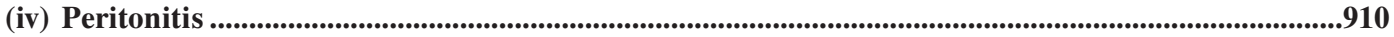

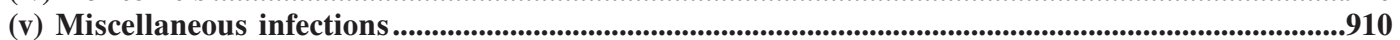

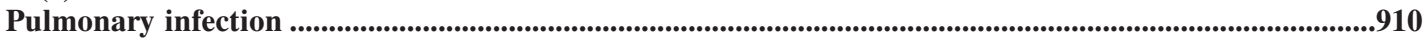

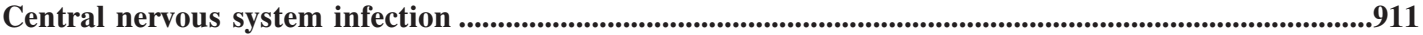

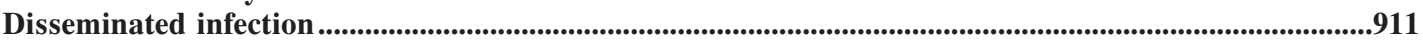

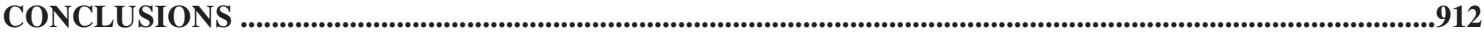

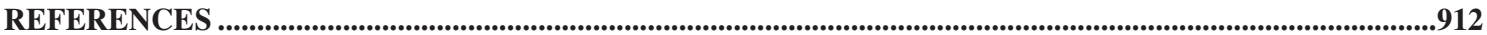

\section{INTRODUCTION}

Melanin is a ubiquitous compound found in many microbes and animals. Its functions are varied but are based on the unique molecular characteristics of its structure, which make it an extremely stable molecule, resistant to a variety of destructive physicochemical processes $(83,109,324)$. In recent years its pathogenic role in fungi has become well described $(123,292$, $375,460,546)$. This review will focus on fungi that are considered to be melanized as a primary feature, particularly with regard to their phenotypic appearance (macroscopic and microscopic morphologies) and appearance in tissue (histology).

The terms used to describe these fungi have evolved over the 
past several decades. As Sporothrix schenckii was one of the earliest melanized fungi described, "sporotrichoid" was often used to describe similar fungi, though currently it has been replaced by other, more useful terms. "Phaeoid," "phaeo-sporotrichose," and "dematiaceous" have also been mentioned in the literature (574). "Phaeo" comes from the Greek meaning "dark" and has been commonly used, particularly when describing infections due to these fungi as "phaeohyphomycosis," i.e., infection caused by dark-walled fungi, as suggested by Ajello et al. (12, 630). It has been suggested that the term "dematiaceous" is not appropriate given its etymologic derivation from the Greek "deme," meaning bundle, though it has become fairly entrenched in medical mycological literature and will likely persist in nomenclature (574). The term "melanized" has become more utilized recently, given its specific meaning. For the purposes of this review, however, the terms "dematiaceous," "melanized," "dark," and "phaeoid" are used interchangeably to denote fungal elements containing melanin.

The presence of melanin alone is probably not a useful criterion for inclusion in this group of clinically important fungi, as melanin has been demonstrated in practically all "nondematiaceous" clinical fungi examined in the literature, including Histoplasma capsulatum, Paracoccidioides brasiliensis, Aspergillus spp., and even Candida albicans (293, 521, 548, 599, 757). One might contrast the fungi discussed here as heavily melanized, with brown-pigmented hyphae in tissue often discernible without the use of any staining procedure. At present, no quantitative measure of melanin is available to distinguish dematiaceous from other fungi. In addition, Sporothrix schenckii, with a yeast form in tissue, is well known and well described (177) as the agent of a unique clinical entity, sporotrichosis, and only issues regarding its mycology will be discussed here.

Melanized fungi are common in the environment (see Ecology below) and are often isolated in the microbiology lab, where they may be considered contaminants. Indeed, only $10 \%$ of dematiaceous lab isolates are likely to have clinical significance $(72,607)$. Clinical disease due to these fungi is uncommon, with one estimate from a large metropolitan area of one case/million persons/year (617). Despite their rarity in clinical practice, melanized fungi have become increasingly recognized as important pathogens, particularly in immunocompromised patients, though individuals with apparently "normal" immune systems have also been reported to have invasive, often fatal infections $(627,628)$.

The clinical syndromes caused by these fungi are differentiated based on histologic findings into eumycetoma, chromoblastomycosis, and phaeohyphomycosis. Eumycetoma is a deep tissue infection, usually of the lower extremities, characterized by the presence of mycotic granules (572). It is associated with a relatively small group of fungi. Chromoblastomycosis is caused primarily by a few species of fungi that produce characteristic sclerotic bodies in tissue and is usually seen in tropical areas (501, 572). Phaeohyphomycosis is a term generally reserved for the remainder of clinical syndromes caused by melanized fungi $(623,630)$. For the purposes of this review, these will be arbitrarily divided into allergic disease, superficial and deep local infections, pulmonary disease, central nervous system (CNS) infection, and disseminated disease. We do not aim to review every publication regarding melanized fungi, but rather we seek to provide a broad, yet in-depth overview of the field as it currently stands, recognizing that it will continue to evolve and expand with our increasing knowledge of and experience with these clinically important fungi.

\section{ECOLOGY}

Melanized or dematiaceous fungi as defined above are frequently considered ubiquitous saprobes inhabiting living and dead plant material and, for the most part, residing in the soil. We now know, however, that these generalized assumptions are incorrect for the group as a whole, as several etiologic agents occupy specific ecological niches or microenvironments, and the knowledge of their natural ecology contributes to our understanding of their opportunistic/pathogenic potential $(175,177,606,779)$. It has been suggested (175) that our use of the term "dematiaceous" be restricted to those ubiquitous, mostly plant-associated hyphomycetous fungi with brown hyphae $(220,221)$, such as Alternaria, Bipolaris, Curvularia, and Exserohilum in the order Pleosporales. The natural ecology of melanized fungi in several other orders is more restricted (187). For example, fungi in the order Calosphaeriales belong mostly to woody-plant- or wood-inhabiting genera such as Phaeoacremonium, Phialemonium, and Pleurostomophora, whereas some genera in the order Chaetothyriales, such as Exophiala, may have specific microenvironments and are characterized as "microextremophiles." The ability of some species in this genus, such as E. xenobiotica, to grow in high concentrations of xenobiotics (606) such as xylene, toluene, or creosote-treated utility poles, as well as to cause human disease, is truly remarkable. Species in the genus Exophiala and related genera are frequently referred to as the "black yeast-like fungi" and are so named because of their ability to produce budding, yeast-like cells at some point in their life cycle as well as dark hyphae. The ecology of Pseudallescheria and Scedosporium was also recently investigated by examining the occurrence of these species in natural and human-dominated environments (393). These findings demonstrated increasing environmental recovery with increasing human habitation and a concomitant elevation in nitrogen concentrations. Another genus defined by its residence in a particular environmental niche is the halophilic genus Hortaea in the order Capnodiales. Hortaea werneckii, the agent of tinea nigra, is found in subtropical saltwater habitats and is manifested by its opportunistic adherence to the dead, salty keratin layers of the human hand (87). Thus, while several genera are considered "ubiquitous," many prefer well-defined microenvironments which, for some genera, predispose them to causing disease where similar conditions exist in the host.

In addition, there are species that appear to be geographically restricted, such as Rhinocladiella mackenziei, which has been seen primarily in patients from the Middle East (726). While Scedosporium prolificans has been reported from many locations, most clinical cases originate from Australia and Spain, for unclear reasons $(76,336)$. This may be due to environmental features that preferentially support specific fungal species.

\section{CLASSIFICATION, TAXONOMY, AND NOMENCLATURE}

Classification of fungi is simply their assignment into defined categories. A classification system is composed of hierarchical 
groups which may be further subdivided to indicate degrees of relationships. The basic unit of classification is the species, although there is currently no universally acceptable definition for this unit. Taxonomy is the arrangement of these fungi into a classification. With multilocus sequencing providing classification insights unavailable to the phenotypic systematists (those who study the relationships and classification of organisms and the processes by which they have evolved), new phylogenetic classification schemes have emerged. Taylor et al. have provided an excellent treatment of the phylogenetic concepts underlying the definition of species in fungi (737). The abbreviated classification scheme to the ordinal level for ascomycetous melanized fungi covered in this review is based upon the most recent work of Hibbett et al. (340) and the Myconet "Outline of Ascomycotya" (463).

Kingdom: Fungi

Phylum: Ascomycota

Subphylum: Pezizomycotina

Class: Dothideomycetes

Orders: Capnodiales, Dothideales, Pleosporales, Botryosphaeriales

Class: Eurotiomycetes

Order: Chaetothyriales

Class: Sordariomycetes

Orders: Microascales, Sordariales, Calosphaeriales, Ophiostomatales

As seen above and in Table 1, clinically significant melanized fungi span several ascomycetous orders in the kingdom Fungi.

Nomenclature refers to assigning formal scientific names. This process is regulated by the International Code of Botanical Nomenclature (ICBN) (http://www.bgbm.org/iapt/nomenclature /code/default.htm) to facilitate a stable naming system and to avoid and reject names which are in error or are ambiguous (789). Lack of adherence to these requisites often invalidates a taxon name and results in multiple names for the same organism. Other reasons for name changes include the placement of fungi into new genera as determined by phylogenetic studies, which frequently occurs within this heterogeneous group of fungi. When this occurs, the species epithet is retained, but it may require modification in keeping with the rules of Latin grammar. An example of recent changes for melanized fungi include the movement of Phialophora richardsiae to Pleurostomophora richardsiae and of Phialophora parasitica to Phaeoacremonium parasiticum. Discovery of a previously unrecognized teleomorph (sexual or meiotic state) may also precipitate a name change. A recent example is found in the discovery of the teleomorph for Scedosporium apiospermum, which was incorrectly thought to be Pseudallescheria boydii. We now know through the work of Gilgado et al. that the teleomorph for $S$. apiospermum is the heterothallic ascomycete Pseudallescheria apiosperma, as evidenced by the production of cleistothecia (round sexual structures containing asci and ascospores) and ascospores (the sexual reproductive propagules) between compatible mating strains of S. apiospermum (283). As the teleomorph name takes precedence over the anamorph (asexual, mitotic) name, the correct binomial would be the sexual state. Whether this name would be adopted by clinicians in everyday usage remains problematic.

Also confusing for clinicians and laboratorians alike is the naming convention that permits the use of more than one name for the same fungus. This is allowed when a particular form of the fungus is the one more commonly seen in the laboratory. Fungi recovered in culture commonly display only an anamorphic state. They may be either heterothallic isolates with no known teleomorph or homothallic strains failing to produce their sexual state in vitro. A few clinically significant homothallic melanized fungi do form both anamorphs and teleomorphs in culture. In this situation, as mentioned above, the teleomorph name takes precedence over the anamorph name, e.g., Pseudallescheria boydii rather than Scedosporium boydii and Microascus cinereus rather than Scopulariopsis cinerea. Additionally, in some genera, such as Pseudallescheria, two separate anamorphs which are distinctively different microscopically may be produced, and these are referred to as synanamorphs (another asexual form of the same fungus). Some homothallic strains, however, lack anamorphs and are known only by the name of the sexual state. Examples would include members of the genus Chaetomium. The advent of sequencing characterization has provided the tools necessary to reevaluate the evolutionary relationships of these black molds, and today multilocus molecular phylogenetic studies are clearly redefining previously described entities, uncovering new species and varieties, and correlating these with their natural habitats.

\section{IDENTIFICATION OF ETIOLOGIC AGENTS}

Over 150 species and 70 genera of dematiaceous fungi have been implicated in human and animal disease (Table 2). As the number of patients immunocompromised as a result of diseases and medical therapy increases, additional species are being reported as causes of human disease, expanding an already long list of potential pathogens. Identification of melanized etiologic agents known to cause human or animal disease has traditionally been based upon phenotypic features of the isolate observed in culture $(175,177,220,221)$. This practice continues to be the mainstay of fungal identification in most routine settings. More recently, molecular techniques employed for classification purposes and those provided by research facilities have provided additional tools for the characterization of these molds. Extensive sequencing for some genera has illustrated the concept of "species complexes," or the inclusion of several separate species into what was formerly referred to as a single species. This has been clearly demonstrated in the genera Exophiala (825), Scedosporium (281-283), and Phaeoacremonium (525). The "splitting" of these species into separate taxa has of necessity changed our reporting practices. As an example, laboratories previously comfortable with discriminating only between Exophiala (Wangiella) dermatitidis and E. jeanselmei are now aware of several other clinically significant species that are not easily separated by phenotypic features alone $(177,184,825)$ and that E. jeanselmei is in fact one of the less frequent agents of disease. Therefore, species other than E. dermatitidis are best reported as an Exophiala sp., not $E$. dermatitidis, unless sequencing has provided a species identification. These "new and improved" reporting techniques, however, must be communicated to clinicians in a manner consistent with their understanding of current organism terminology and the associated mycoses. 


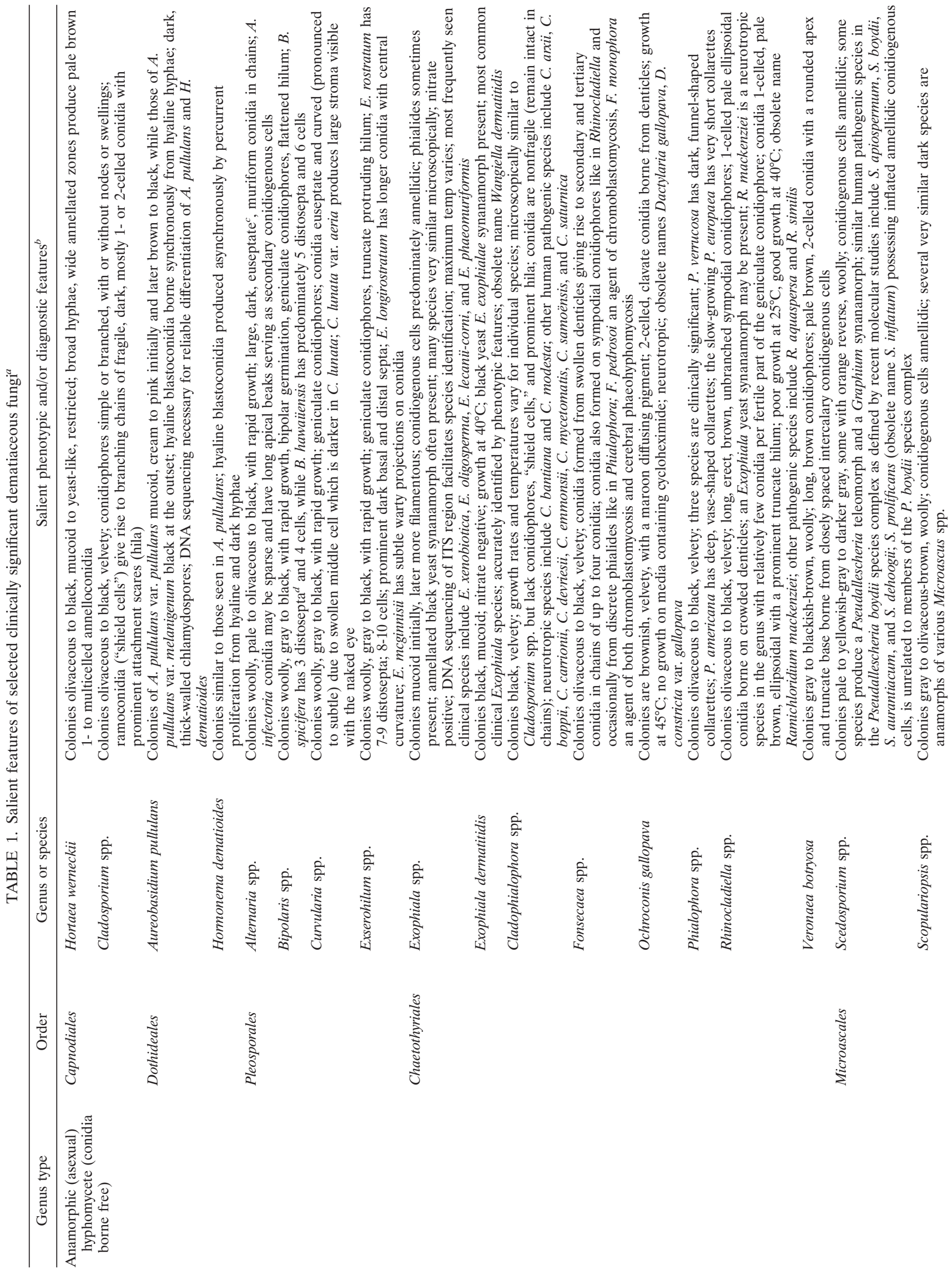




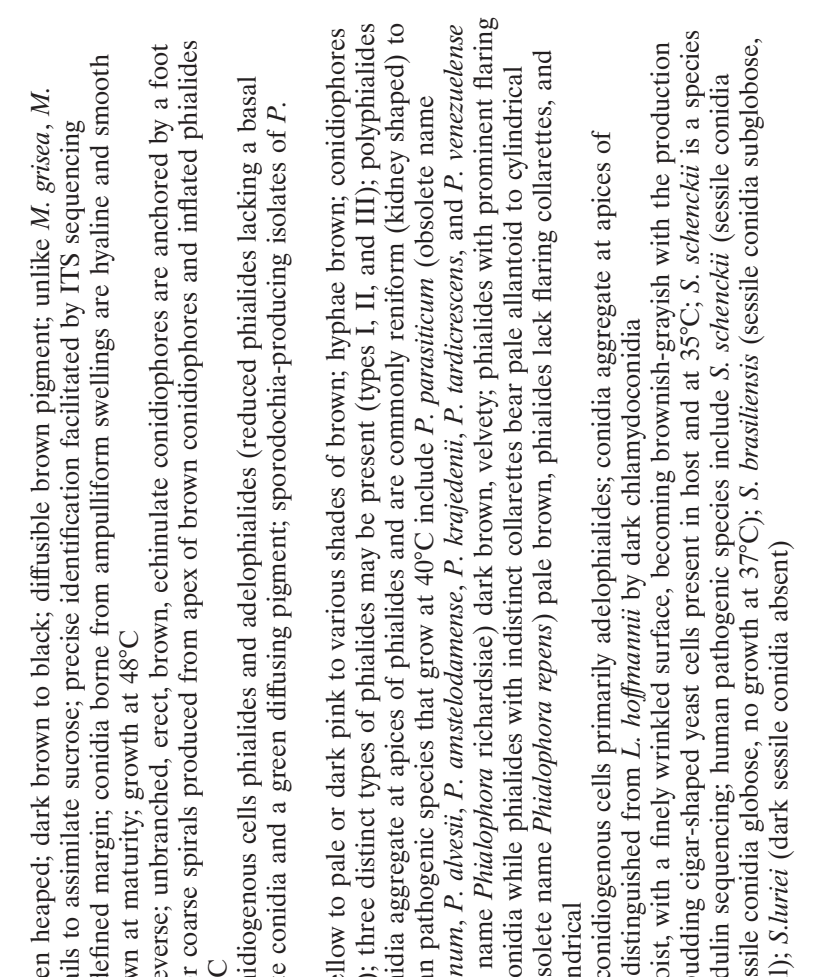
Hath Hat

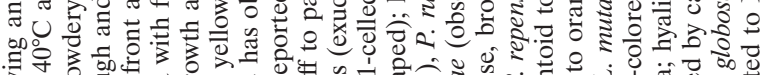

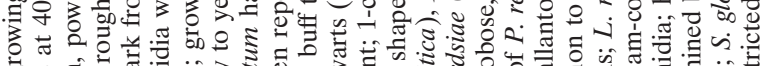

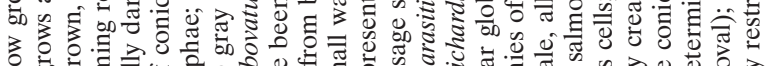
Muntwy

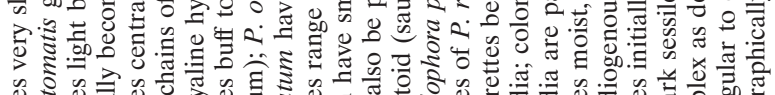

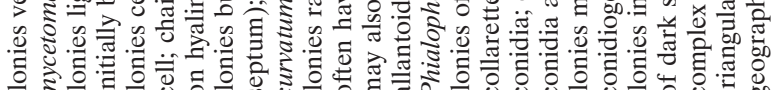
Why
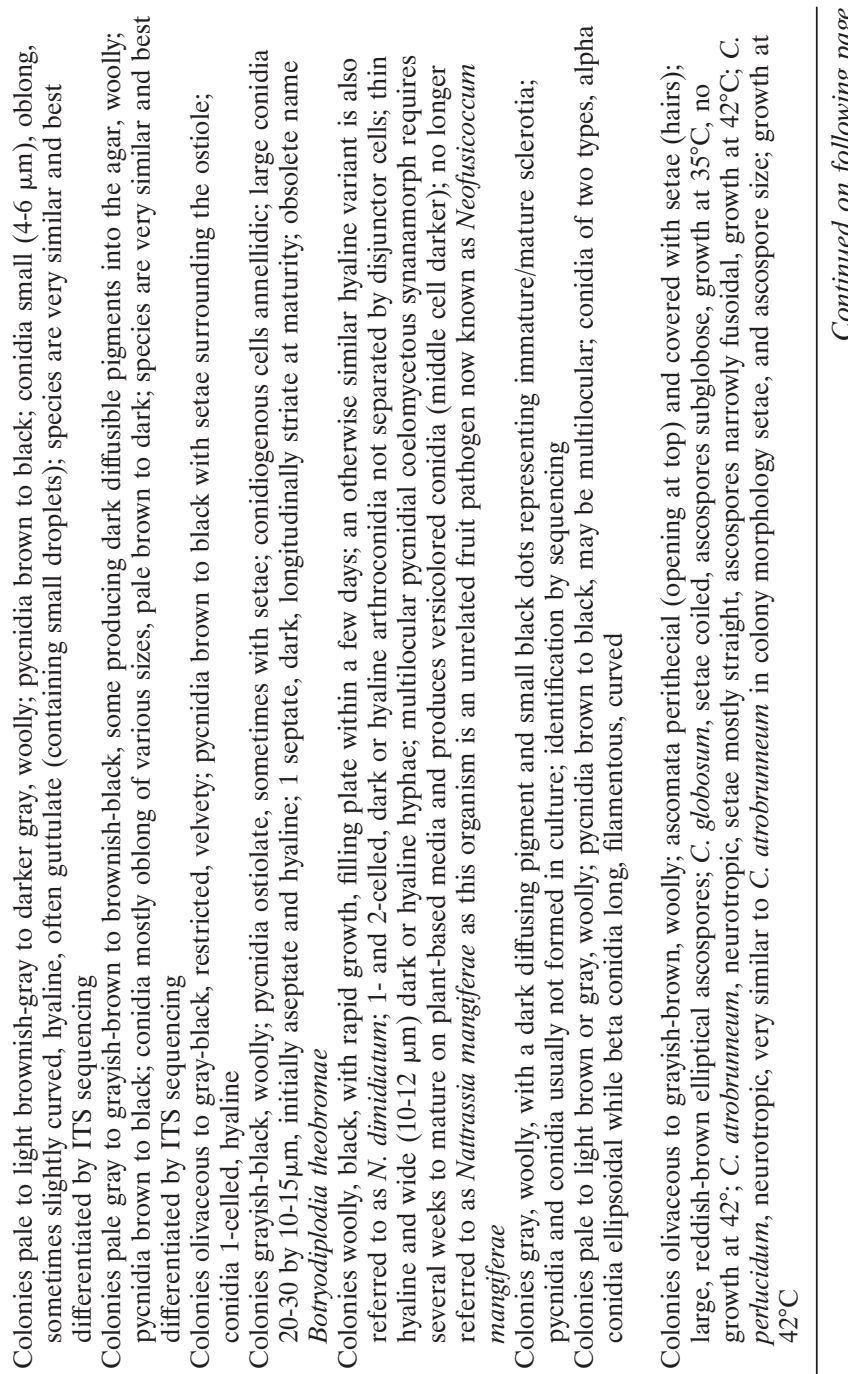

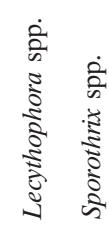

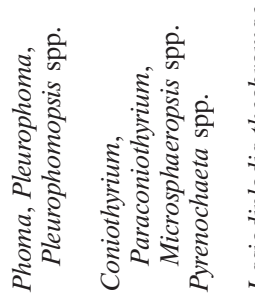
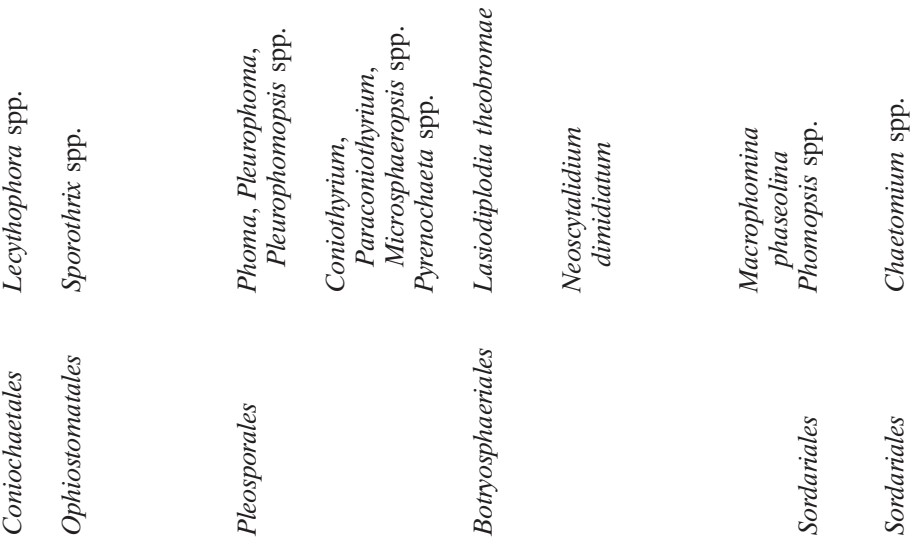
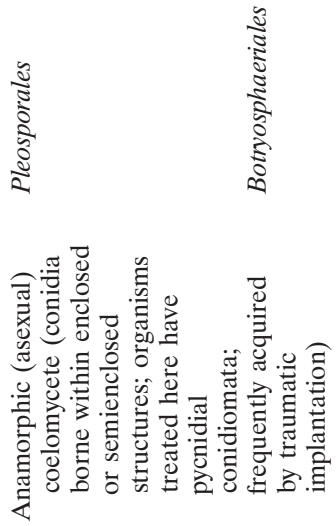

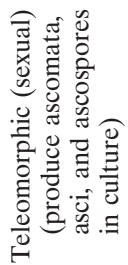




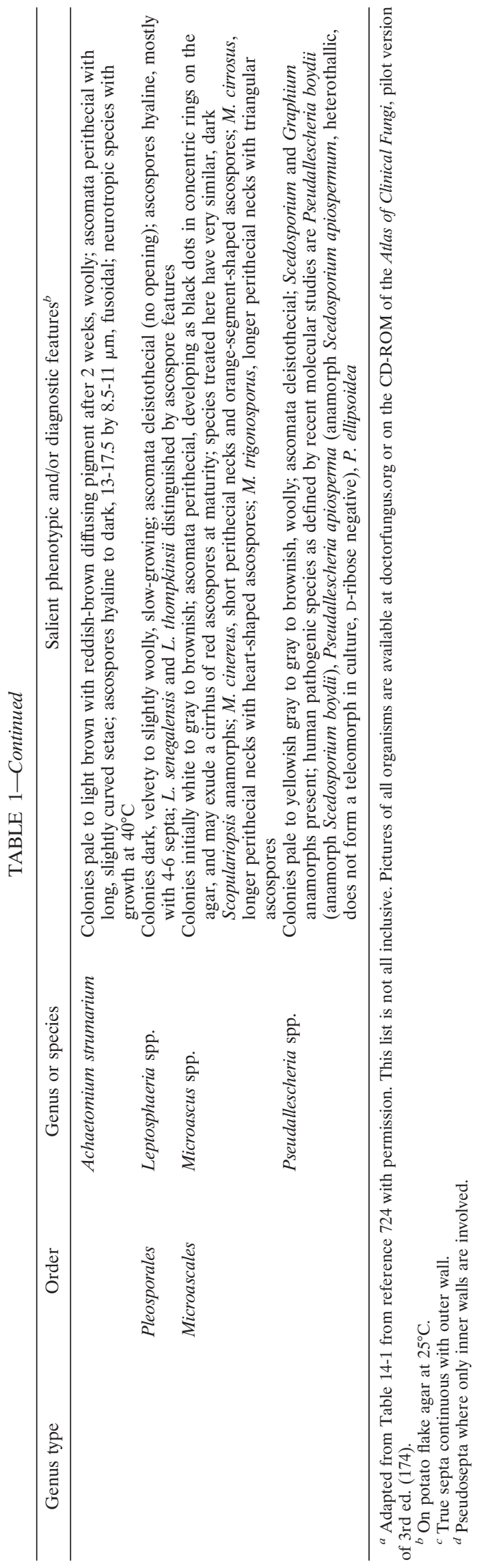

\section{Phenotypic Identification}

The level to which black molds can or should be identified in the routine laboratory may depend on several factors, such as the genus of the organism recovered, whether or not an epidemiologic investigation is warranted, and/or the level of identification required for appropriate patient management. The phenotypic identification of black molds is based primarily upon their macroscopic morphology (color, growth rate, and growth characteristics on standardized media), their microscopic morphology (hyphae, conidiogenous cells [specialized cells that produce the conidia], conidia [asexual reproductive propagules], etc.), and a limited number of physiologic features (primarily cycloheximide tolerance, nitrate assimilation, urea hydrolysis, and growth at various salt concentrations). Only genus-level identification may be possible or required for genera with several similar, closely related species, such as described above for Exophiala. In some other genera, certain species are clearly associated with a particular type of mycosis, and a combination of morphologic features, temperature, and physiology can provide a species-level identification. This is the case for the agent of cerebral phaeohyphomycosis, Cladophialophora bantiana.

Macroscopic morphology. The medium (see "Isolation Procedures and Culture" in Diagnosis below) is an important consideration in the identification of melanized fungi. The use of a medium that promotes growth most consistently matching the original description of the organism is preferred, and this typically is a plant-based medium. The most commonly used is potato dextrose agar (PDA) or variations thereof. It provides colony colors that are close to those originally described, and it is usually adequate for conidiation. Other plant-based media include malt extract agar, V-8 juice agar, cereal agar, carnation leaf agar, cornmeal dextrose agar, and others. A more complete list of media and reagents may be found in the Manual of Clinical Microbiology, 9th ed. (441), and in the Atlas of Clinical Fungi, 2nd ed. (175). Phaeoid molds vary considerably in their colony colors. Although this characteristic is highly dependent upon environmental conditions, it is one that can be useful in the initial separation of genera/species. While most species are various shades of pale gray to dark gray to black, others may be brown or very pale or may turn darker only with the production of certain structures. Others may be some shade of purple or distinguished by diffusible pigments. Etiologic agents which are typically brown on PDA include Ochroconis gallopava, Pleurostomophora richardsiae, Pleurostomophora repens, some Phaeoacremonium species, Wallemia sebi, Myceliophthora thermophila, and Veronaea botryosa. The "pale list" includes fungi which seldom turn dark, such as Phialemonium species. Lecythophora mutabilis remains lightly colored until the production of dark chlamydospores. Ochroconis gallopava exudes a winered pigment into the agar (more pronounced on Sabouraud dextrose agar [SDA]), and several Phaeoacremonium species exhibit purple to lavender colonies.

Microscopic morphology and pleomorphism. Variable microscopic morphology in the same fungus, also referred to as pleomorphism or pleoanamorphism, is another feature useful in the phenotypic identification of black molds. Some fungi may display more than one form, such as yeast-like growth initially and more filamentous growth subsequently. This is 
TABLE 2. Melanized fungi in human disease ${ }^{a}$

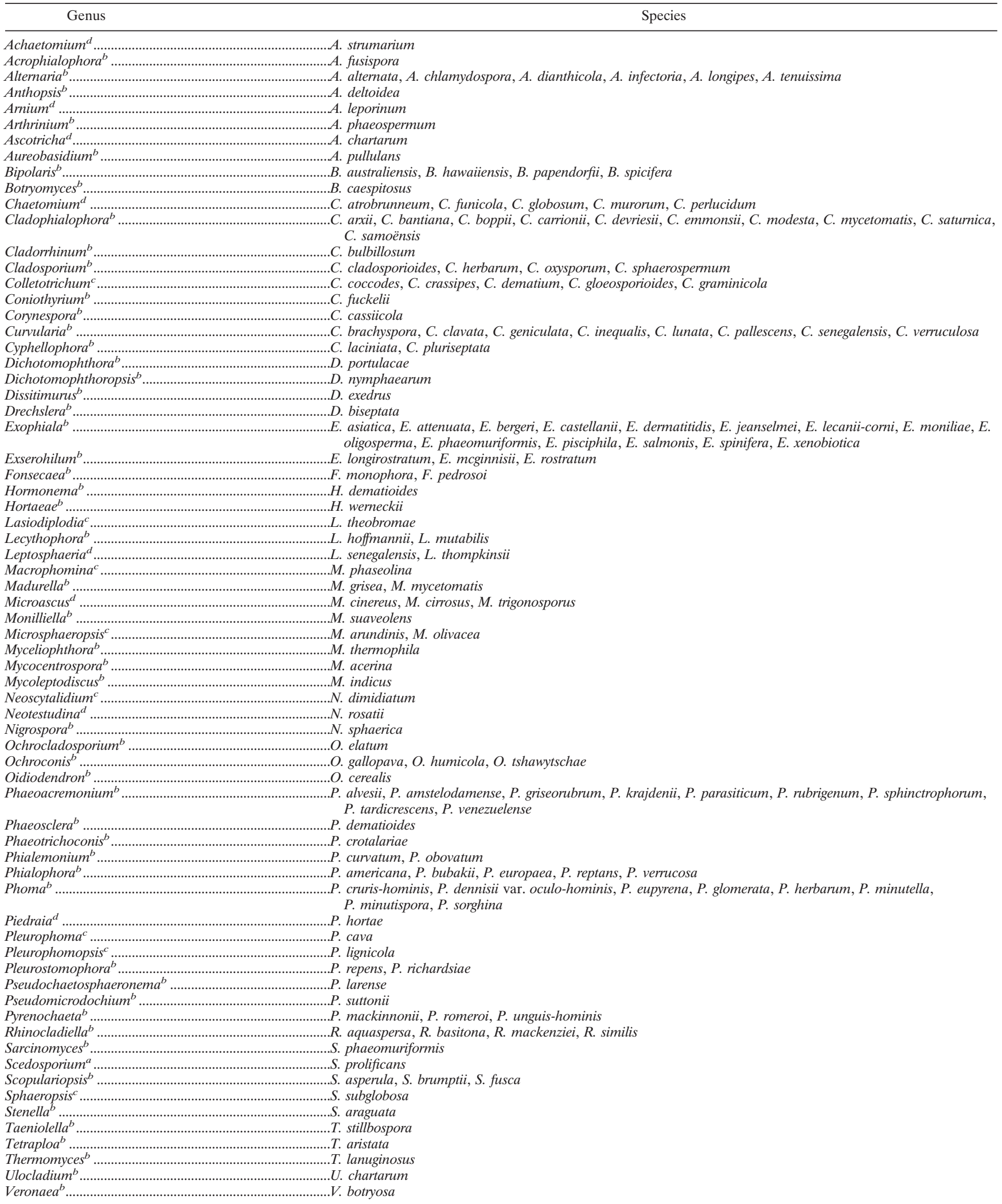

${ }^{a}$ Some doubtful cases have been omitted; the list may not be all inclusive. Some genera that are outside the taxonomic orders discussed in the text but that contain melanized structures are included. Adapted from reference 493 with permission of the publisher.

${ }^{b}$ Anamorphic hyphomycete.

${ }^{c}$ Anamorphic coelomycete.

${ }^{d}$ Teleomorphic ascomycete. 


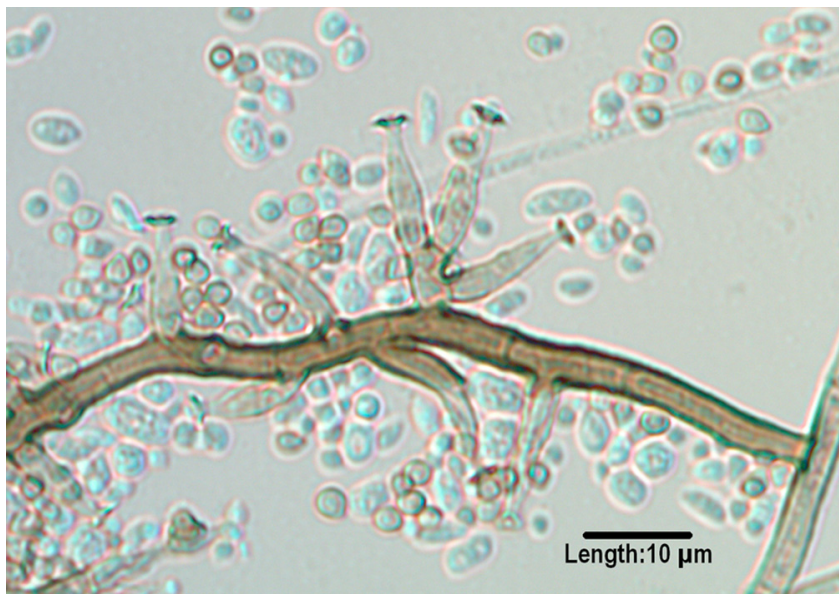

FIG. 1. Conidiogenous cells of Pleurostomophora richardsiae, demonstrating prominent flaring collarettes as well as the two types of conidia (oval and globose) produced by this species. (Unless otherwise noted, in this and subsequent figures light microscopy photomicrographs of conidiogenous cells and/or conidia were taken from slide culture preparations grown on potato flakes agar for 7 days at $25^{\circ} \mathrm{C}$.)
Physiologic features. Physiologic characteristics may also assist in separation of various genera/species. However, only those that are available in routine laboratories are widely employed. The ability or inability of isolates to grow on media containing cycloheximide (referred to as cycloheximide tolerance), nitrate assimilation, urease activity, and salt tolerance, particularly for halophilic strains, are all useful adjuncts to the morphologic examination. Larger reference labs and research facilities also may use a battery of carbon assimilation profiles. Temperature tolerance is also useful in segregating potential pathogens. Those that fail to grow at $35^{\circ} \mathrm{C}$ are more likely to be recovered from superficial sites, while those capable of growth at this temperature have the potential for more invasive human disease. Several clinically significant dematiaceous molds are thermotolerant to thermophilic, with maximum growth temperatures to $45^{\circ} \mathrm{C}$ and beyond. A partial listing of these potentially neurotropic species includes $E$. dermatitidis, $O$. gallopava, C. bantiana, C. modesta, C. emmonsii, R. mackenziei, Acrophialophora fusispora, Fonsecaea monophora, and some aggressive Achaetomium and Chaetomium species.

\section{Molecular Characterization}

common in the black yeasts such as Exophiala and related genera. Pleoanamorphism may also be exhibited by different types of anamorphic structures (synanamorphs), such as the Graphium state in Pseudallescheria or variably shaped conidia in Pleurostomophora richardsiae (Fig. 1). Identification of homothallic ascomycetes is typically based on the type of ascomata produced (primarily cleistothecia [round, closed structures containing asci and ascospores] or perithecia [round to pear-shaped structures with an opening or ostiole containing asci and ascospores], as in Pseudallescheria or Chaetomium/ Achaetomium/Microascus, respectively) and differences in ascospore morphology. Ascospores may be of various sizes, shapes, colors, and ornamentations. The bulk of clinical black molds, however, are heterothallic ascomycetes. These mitosporic fungi are identified mostly by their methods of conidiogenesis and the morphology of their conidia. The majority of mitosporic isolates are hyphomycetes with their conidia borne free in the aerial mycelium. Also seen are coelomycetes, whose conidia are borne within asexual structures known as conidiomata. The methods of conidiogenesis (blastic [blown-out = blastoconidia, as seen in many genera] or thallic [formed from preexisting hyphae $=$ arthroconidia, as in Neoscytalidium]), the types of conidiogenous cells (primarily annellidic [Scedosporium, Scopulariopsis, and Hortaea] or phialidic [many genera]), and the morphology of the conidia are taken in aggregate to form the basis for a morphologic identification. Annelloconidia are formed from percurrent, indeterminate conidiogenous cells that produce rings or annellations and become longer and narrower with the production of conidia, while phialoconidia are formed from conidiogenous cells with collarettes that may be quite distinct or subtle, and the conidiogenous cell remains the same size and shape with conidial production. It should be pointed out, however, that these morphologic features used to identify anamorphic species lacking teleomorphs are strictly phenotypic and do not define their phylogenetic placement within the order (157).

Molecular characterization of fungi is a mature discipline in the molecular systematics arena, with multilocus datasets, extensive taxon sampling, and rigorous analytical methods being the norm (340). Its use in the clinical laboratory, however, is mostly restricted to epidemiologic studies and to identification of unusual/uncommon or difficult-to-identify isolates. Molecular identification of most species relies on sequencing of ribosomal genes and comparison with published databases, notably those in GenBank; however, over $10 \%$ of these deposits may be erroneous (176). Private databases are also sometimes utilized for particular genera; however, these are difficult to access and may also contain incorrect deposits. Also, various methods and genes or gene regions such as the internal transcribed spacer regions ITS1 and ITS2, the D1/D2 domains, $\beta$-tubulin, actin, calmodulin, manganese superoxide dismutase, ATPase subunit 6 , chitin synthase, mitochondrial small-subunit (SSU) rRNA, translation elongation factor $1 \alpha$, and others are utilized, so that interlaboratory standardization of sequencing is lacking. Several International Society for Human and Animal Mycology (ISHAM) working groups are addressing standardization of fungal sequencing (58) as is the Clinical and Laboratory Standards Institute (CLSI) (146). Genera for which substantial sequencing data are available and for which species distinction appears to be satisfactory include those known as black yeasts, i.e., Exophiala and related genera (ITS) (825), Sporothrix species (calmodulin) (480), Phaeoacremonium species ( $\beta$-tubulin and actin) (525), and Pseudallescheria/Scedosporium species (ribosomal DNA [rDNA] gene cluster, $\beta$-tubulin, calmodulin, and translation elongation factor $1 \alpha$ ) (330). Molecular characterization should always be evaluated in light of phenotypic features, and sequence data for uncommon and/or potentially new species should be compared with those for ex-type strains. 


\section{ANAMORPHIC HYPHOMYCETE GENERA}

\section{Capnodiales}

Hortaea. Hortaea werneckii is the etiologic agent of tinea nigra, an asymptomatic, superficial mycosis causing hyperchromic plaques without keratinolysis in the dead keratin layers of the skin $(186,685)$ and mostly restricted to the palms of the hands (tinea nigra palmaris) and soles of the feet (tinea nigra plantaris) (87). It is a halophilic organism whose natural habitat is in tropical and subtropical hypersaline environments (823), and it is thought be acquired through superficially abraded skin (186). Colonies are restricted, black, moist, and yeast-like initially, later becoming filamentous. Wide hyphae are densely septate, thick walled, and brown. Intercalary or lateral annellidic conidiogenous cells produce brown, twocelled ellipsoidal conidia with a darkened central septum. ITS sequencing facilitates molecular identification (823) and clearly distinguishes $H$. werneckii from other closely related halophilic and acidophilic (H. acidophila) nonpathogenic species (347).

Cladosporium. The genus Cladosporium has recently undergone molecular and morphologic scrutiny (157), with many organisms being reassigned to other genera. One example is the transfer of Cladosporium elatum to Ochrocladosporium elatum. The genus is extremely ubiquitous, and although it is an agent of allergic disease in indoor settings, few species are documented to cause disease. The species complexes Cladosporium cladosporioides and $C$. oxysporum are the ones most commonly cited in cases of cutaneous and subcutaneous disease $(313,586,641,776)$ and occasionally deeper infections $(396,429)$; however, they are commonly contaminants, making the nature of reports doubtful. The inability of Cladosporium species to grow on media containing cycloheximide, their prominent "shield cells," and conidia that are fragile (easily detached) and possess dark hila (attachment scars) are all features distinguishing Cladosporium from the unequivocally pathogenic Cladophialophora species.

\section{Dothideales}

Aureobasidium. Recent molecular characterization of $\mathrm{Au}$ reobasidium pullulans and closely related organisms by multilocus sequence analysis (ITS, partial 28S rDNA, $\beta$-tubulin, translation elongation factor $1 \alpha$, and elongase), expanding the work of de Hoog and Yulova and of Yulova et al. (173, 820 ), has shown that the genus Aureobasidium contains a single species and several varieties containing differing amounts of melanin and having various salt $(820,823)$ and temperature (824) tolerances. The mode of conidiogenesis is primarily synchronous rather than percurrent, as in Hormonema; however, features of conidiogenesis are difficult to ascertain with certainty. Sequencing is usually required for a definitive identification. Two varieties are human pathogens, $A$. pullulans var. pullulans, and $A$. pullulans var. melanigenum. In the former, colonies remain pink for approximately 1 week, tolerate $15 \%$ salt, and have a maximum temperature of $30^{\circ} \mathrm{C}$, while in the latter, colonies are black at the outset, tolerate $10 \%$ salt, and have a maximum temperature of $35^{\circ} \mathrm{C}$. Aureobasidium is an opportunistic pathogen of humans and animals recovered in cases of catheter- related septicemia $(117,360)$, disseminated infections $(344$, 663), chromoblastomycosis (616), and peritonitis $(144,367)$.

Hormonema. As noted above, Hormonema species are phenotypically similar to Aureobasidium pullulans; however, conidiogenesis is primarily percurrent rather than synchronous. There are rare reports of cutaneous phaeohyphomycosis (149) and fungal peritonitis (690) due to this organism, both of which were reported prior to molecular characterization.

\section{Pleosporales}

Alternaria. Alternaria is a large genus of plant pathogen species that are only occasionally implicated in opportunistic human disease. Cutaneous and subcutaneous phaeohyphomycosis in immunosuppressed individuals is the most common presentation $(275,577,587,798)$. Organ transplantation (280) and Cushing's syndrome appear to be major risk factors for cutaneous/subcutaneous disease, while bone marrow recipients are at risk for sinusitis (577). Ocular disease in individuals exposed to soil and garbage (577) is the next most common presentation, while onychomycosis is rarely reported. There are also occasional reports of allergic fungal sinusitis (67) While several species, such as $A$. chlamydospora $(65,703), A$. longipes (275), and $A$. tenuissima (124, 642, 644), have been reported, most clinical isolates have been shown to be either $A$. alternata $(176,497,710)$ or $A$. infectoria $(99,551,648)$. ITS region sequences have demonstrated that $A$. longipes and $A$. tenuissima cannot be distinguished from $A$. alternata. Conidial production by Alternaria infectoria is sparse, and colonies may be pale.

Bipolaris. The most common mycosis attributed to Bipolaris spp. is allergic fungal sinusitis (125, 246, 417, 444, 508, 580). Other disease associations include subcutaneous lesions, keratitis, and peritoneal dialysis-associated peritonitis (508). Extension to the central nervous system via the nasal sinuses highlights the neurotropic potential of the genus, though this is very rare $(260,817)$. Clinically significant species inciting human disease include B. spicifera, B. hawaiiensis (Fig. 2), and $B$. australiensis. They are differentiated morphologically by conidial size and the number of distoseptations (pseudosepta where only inner walls are involved) (20). Conidia demonstrate bipolar germination, hence the genus name "Bipolaris."

Curvularia. Curvularia species are common in dead plant material and may cause a variety of human mycoses, including fungal keratitis, invasive sinusitis (215), onychomycosis, black grain eumycotic mycetoma (378), endocarditis (104), subcutaneous disease $(813)$, and peritonitis $(98,241,631)$ as well as systemic infections $(175,177)$. Additional reports involved fatal cerebral phaeohyphomycosis in an immunocompetent individual (121), endophthalmitis (579), and contaminated salinefilled breast implants (392). Clinical isolates include $C$. geniculata, C. lunata, $C$. pallescens, $C$. senegalensis, $C$. brachyspora, C. clavata, C. verruculosa, and C. inaequalis (Fig. 3) (598). $C$. lunata is the most common clinical species, and $C$. lunata var. aeria (Fig. 4) may produce large, upright stroma in culture that are visible with the naked eye.

Exserohilum. Three Exserohilum species recovered from humans are E. rostratum, E. longirostratum, and E. mcginnisii, although molecular studies suggest that they may be the same species $(175,177)$. The genus is characterized by its long, 


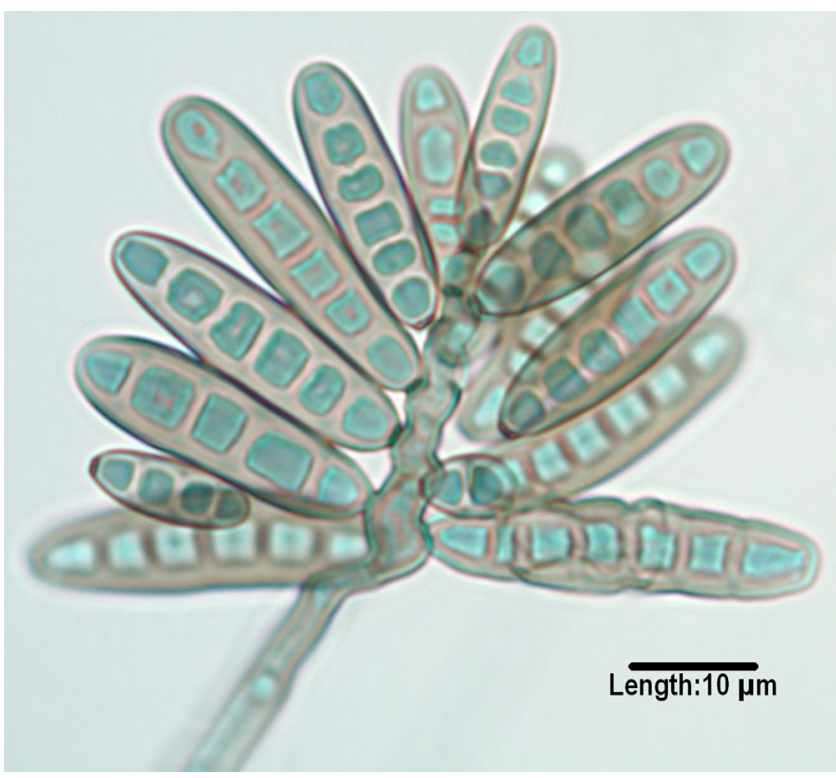

FIG. 2. Conidia of Bipolaris hawaiiensis, demonstrating mostly five distosepta and six cells being borne from a geniculate conidiophore/ conidiogeous cell.

multidistoseptate conidia and a protruding hilum. E. rostratum exhibits darkened basal and distal septa, and E. longirostratum has conidia that are noticeably longer and centrally curved (Fig. 5), while E. mcginnisii has conidia with warty projections on their outer walls. Not all authorities agree that E. rostratum

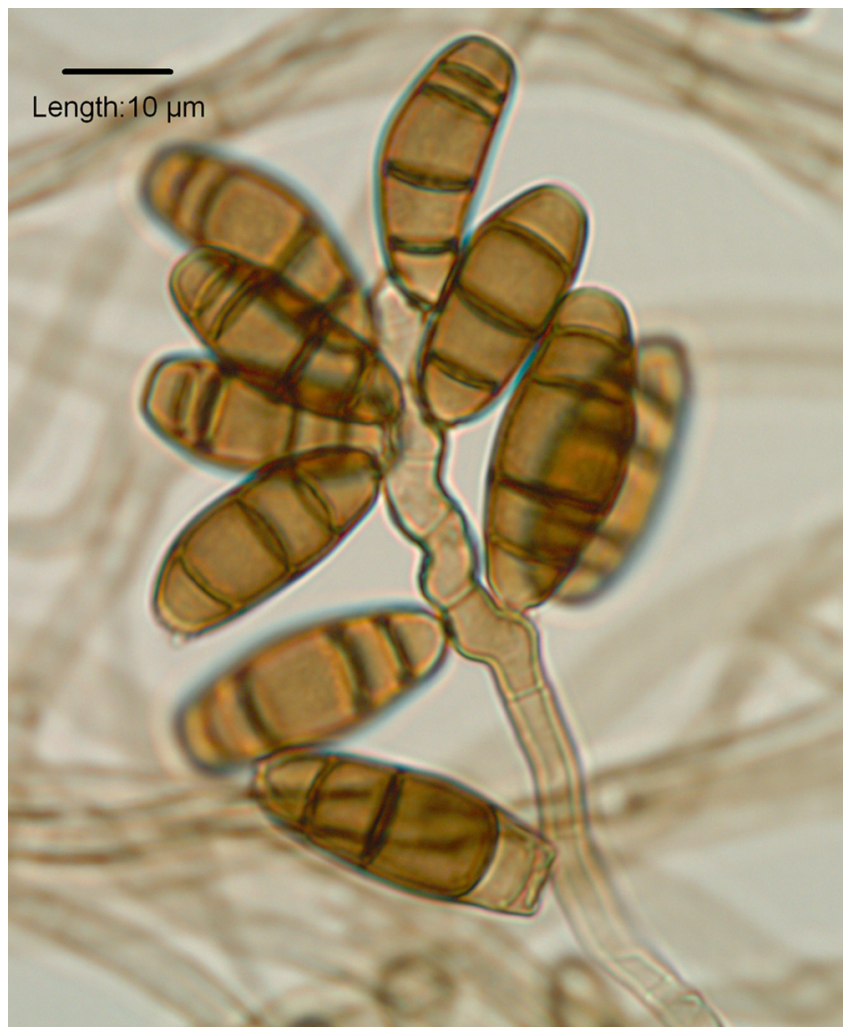

FIG. 3. Conidia of Curvularia inaequalis with mostly five septa and six cells borne from a geniculate conidiogenous cells.

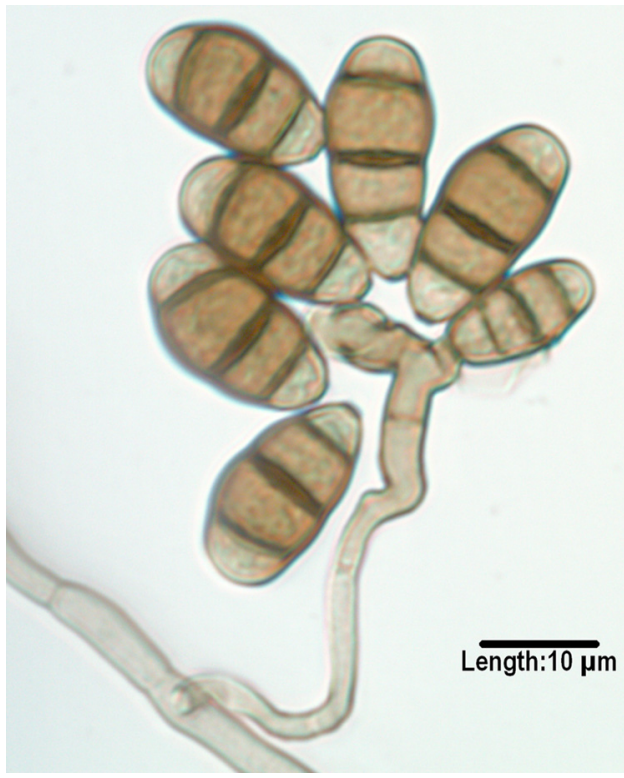

FIG. 4. Conidia of Curvularia lunata var. aeria borne from a geniculate conidiogenous cell. Note that the middle cell is slightly enlarged, and septa are eusepta (true septa continuous with the outer wall).

and E. longirostratum are separate species. Species are opportunistic and are etiologic agents of sinusitis (566), which may extend to the central nervous system (46), and keratitis (488), as well as cutaneous and subcutaneous mycoses $(359,508,580)$. A fatal disseminated case was reported in a patient with aplastic anemia (37).

\section{Chaetothyriales}

Exophiala. Species in the genus Exophiala are frequently referred to as "black yeasts" due to the ability of several species to form a budding yeast-like synanamorph as well as hyphal forms. Colonies are olivaceous-black with a black reverse and are initially moist or yeast-like, later becoming velvety at maturity. Asexual replication is by annellidic conidiogenous cells, and conidia are formed in clusters both from intercalary

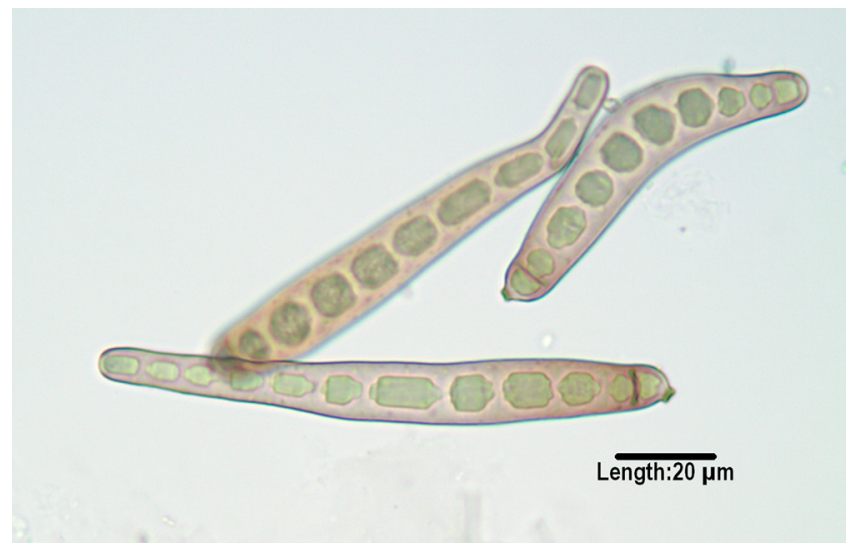

FIG. 5. Multidistoseptate conidia of Exserohilum longirostratum, demonstrating a prominent basal septum (true septum) and a protruding hilum. 


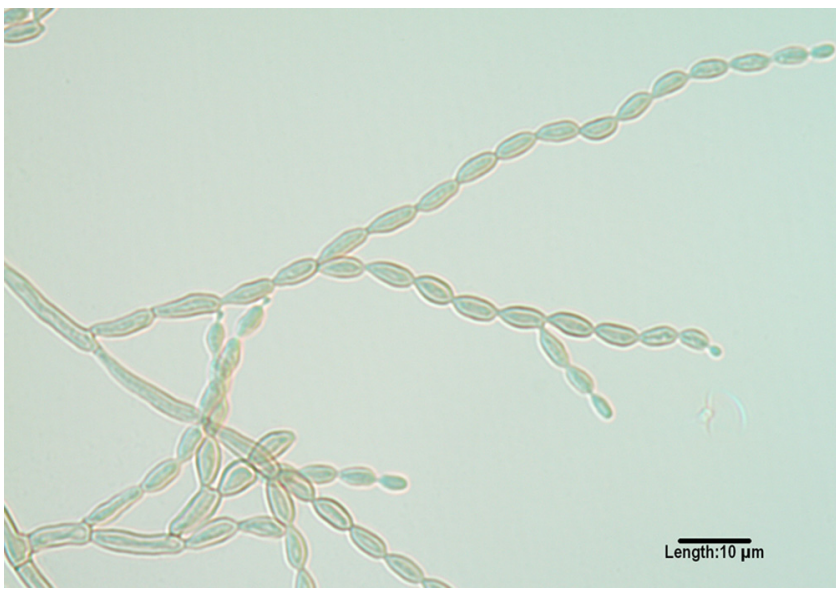

FIG. 6. Long, nonfragile chains of conidia as seen in Cladophialophora bantiana.

conidiogenous loci and at the tips of annellides. Some species may occasionally form conidia in chains (182) (catenate) on nutritionally deficient media or display phialides as well as annellides (183). Species are very similar microscopically, and unequivocal differentiation is facilitated by physiologic features such a temperature tolerance and nitrate assimilation and by molecular characterization. Some waterborne psychrophilic species such as E. pisciphila are pathogens of fish $(436,438)$, while others such as E. mesophila are found in dental unit water lines (604) and municipal drinking water (300). The most clinically important species are thermotolerant (719). In a recent study of U.S. clinical isolates, reidentification of strains by ITS sequencing showed the most common species to be $E$. dermatitidis (29\%), E. xenobiotica (20\%), and E. oligosperma $(19 \%)(94,825)$. While many clinical isolates are reported as $E$. jeanselmei, which has been regarded as a major agent of subcutaneous phaeohyphomycosis, this species made up only $8 \%$ of the isolates, and molecular studies clearly showed $E$. jeanselmei to be a heterogeneous complex of species $(184,780)$. Exophiala jeanselmei has been redefined clinically as an agent of traumatic cutaneous infection eventually leading to eumycetoma $(27,52,683)$. Exophiala dermatitidis is distinguished phenotypically by its mostly mucoid colonies, ability to grow at $40^{\circ} \mathrm{C}$, lack of nitrate assimilation (569), and yeast cells surrounded by capsules (819), which it shares with another aggressive species, E. spinifera $(180,214,536,699)$. The range of mycoses incited by $E$. dermatitidis include neurotropic infections in young, immunocompetent individuals (restricted to Asia) $(138,345,492,494)$, systemic lymphadenitis (13), cutaneous and subcutaneous infections in mostly immunocompromised individuals $(346,492)$, colonization of airways in cystic fibrosis patients (597), and mycoses related to continuous ambulatory peritoneal dialysis (CAPD) (783). It is also an opportunist in lungs of cystic fibrosis patients $(320,355)$ and may be recovered from the stool in patients with diarrhea (178). It has been recovered from Turkish steam baths (489) and associated with free-living amoebae in hospital water (128). E. phaeomuriformis, which is similar in morphology to E. dermatitidis, can grow at a maximum temperature of $38^{\circ} \mathrm{C}$ (490). Exophiala spinifera and the similar E. attenuata (780) have long, spine-like

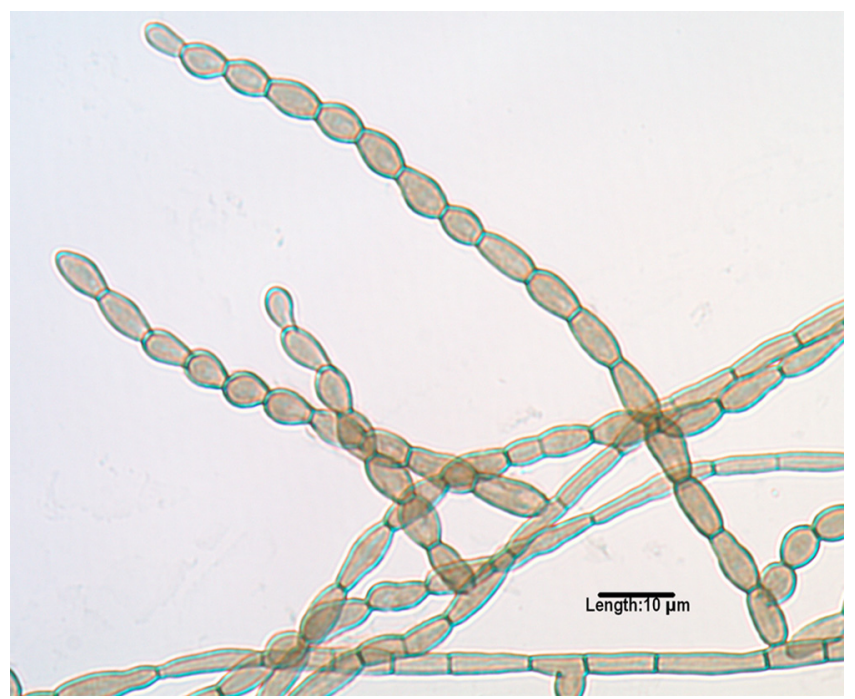

FIG. 7. Long, nonfragile chains of conidia produced by a less common species of Cladophialophora, C. emmonsii. Note that conidiophores and prominent hila (attachment scars) are absent.

conidiophores. E. spinifera is an agent of serious disseminated mycoses in adolescents (180) and of cases of subcutaneous phaeohyphomycosis $(327,699)$. E. xenobiotica, which is capable of growing in the presence of high concentrations of xenobiotics such as xylene, toluene, or creosote-treated utility poles, was the agent of subcutaneous phaeohyphomycosis in a nonHodgkin lymphoma patient (36). E. asiatica is a newly described species causing a fatal, disseminated cerebral phaeohyphomycosis in China (452).

Cladophialophora. Cladophialophora species, although morphologically similar to Cladosporium species, are differentiated by belonging to a different order, the Chaetothyriales rather than the Capnodiales; by lacking conidiophores, "shield cells," or prominent hila (attachment points); by their ability to grow on media containing cycloheximide; and by having dry, nonfragile chains of conidia. The genus has recently been reevaluated by multilocus sequencing and currently contains seven species associated with humans (51). C. bantiana, (Fig. 6), previously characterized at the molecular level (279), is a neurotropic species with growth at $40^{\circ} \mathrm{C}$ and is the causative agent of numerous cases of cerebral phaeohyphomycosis $(204,272$, $353,395,628,733)$, many of which occur in immunocompetent individuals and most of which are fatal. The species has also been reported as an agent of eumycetoma (89), along with Madurella mycetomatis (51). Less common species occasionally incriminated in deep and superficial mycoses include $C$. modesta, C. arxii, C. devriesii, C. emmonsii (Fig. 7), C. boppii, and $C$. saturnica $(47,51,295,505,516,568,748)$. C. carrionii and the recently described $C$. samoënsis are agents of chromoblastomycosis $(51,229,446,610,826)$. C. yegresii is considered a closely related environmental sister species to C. carrionii (181, 782).

Fonsecaea. The genus Fonsecaea is comprised of two species $(174,533) . F$. pedrosoi is known almost exclusively as an agent of chromoblastomycosis $(45,515,610,695)$, while the newly described $F$. monophora (Fig. 8 ) is known as an agent of chromoblastomycosis $(808,809,810)$ and subcutaneous disease 


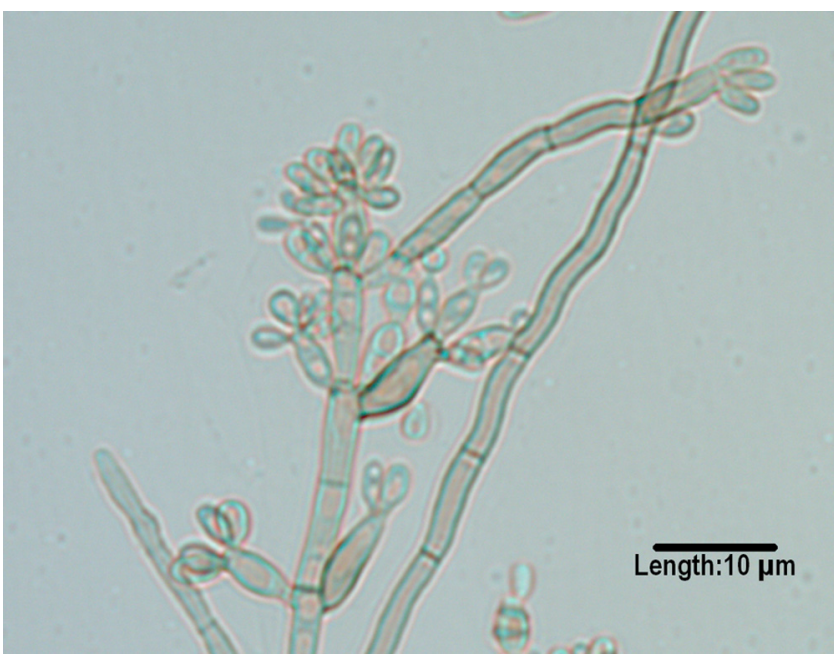

FIG. 8. Conidial formation in Fonsecaea monophora. Conidia are formed from swollen denticles which give rise to secondary and tertiary conidia in chains of up to four conidia. The same type of conidiogenesis occurs in F. pedrosoi.

and, more recently, cerebral phaeohyphomycosis (721, 733). Prior reported cases of central nervous system and/or other deep tissue infections $(520,545,701)$ should most likely be attributed to $F$. monophora. A murine model of disseminated infection with $F$. monophora was recently reported (113). Both species form conidia from swollen denticles which give rise to secondary and tertiary conidia in short chains of up to four conidia. Conidia may also be formed from sympodial conidiophores, as in Rhinocladiella, and in balls from discrete phialides with collarettes, as in Phialophora. Molecular characterization is required for unequivocal differentiation.

Ochroconis. O. gallopava was initially observed to cause central nervous system disease in poultry (354). It has subsequently been shown to be an etiologic agent of neurotropic infections in immunocompromised humans (692) as well as pulmonary infections in immunocompetent hosts $(348,554)$. O. gallopava has colonies that are brownish rather than gray or olivaceous, produces a maroon diffusing pigment more pronounced on SDA than on PDA, grows at $40^{\circ} \mathrm{C}$, fails to grow on media containing cycloheximide, and displays clavate, twocelled, hyaline conidia borne on long denticles (Fig. 9).

Phialophora. Some human pathogens with phialidic conidiogenesis previously assigned to Phialophora (263) have been moved to other genera, namely, Phaeoacremonium (525) and Pleurostomophora (777), leaving only those species that are filamentous throughout their life cycle. Both $P$. verrucosa and $P$. americana produce their conidia from phialides with conspicuous darkened collarettes; these are funnel shaped and vase shaped in $P$. verrucosa (Fig. 10) and P. americana (Fig. 11), respectively. Sequencing has demonstrated a close relatedness, suggesting that the species may be synonymous (185, 811). $P$. verrucosa is primarily an agent of chromoblastomycosis (257, 770), although other reported infections include endocarditis, keratitis, and osteomyelitis $(209,760)$. A recently described species implicated in superficial infections, $P$. europaea, has very short collarettes (179).

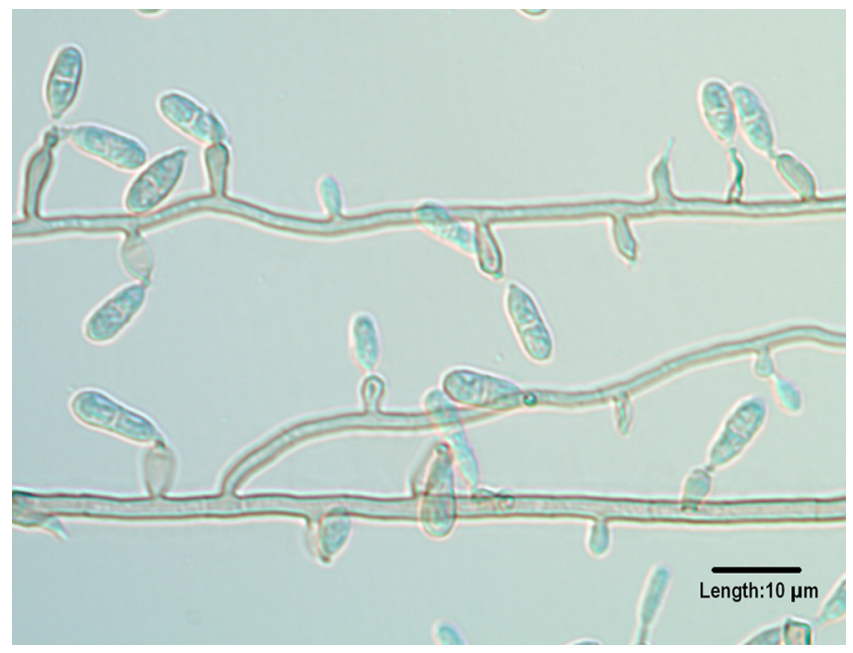

FIG. 9. Two-celled, clavate (club-shaped) conidia of Ochroconis gallopava borne on long, thin denticles.

Rhinocladiella. Four species of Rhinocladiella are known agents of human disease. $R$. mackenziei (formerly Ramichloridium mackenziei and also thought to be synonymous with Ramichloridium obovoideum, which is now unrelated in the genus Pleurothecium as $P$. obovoideum) (43) is a frequently fatal neurotropic organism previously thought to be restricted to individuals residing in or immigrating from Middle Eastern countries $(114,394,726)$. It has now been reported as the etiologic agent of a brain abscess in a man from India, an area where it is not endemic, who reported no travel outside the country (48). $R$. aquaspersa is an occasional agent of chromoblastomycosis $(39,589,693)$. $R$. basitona was recovered from subcutaneous lesions in a man from Japan (43). R. similis (184) appears to be the agent reported under the name $R$. atrovirens in cases of mycetoma (535) and cerebral phaeohyphomycosis in an AIDS patient (193).

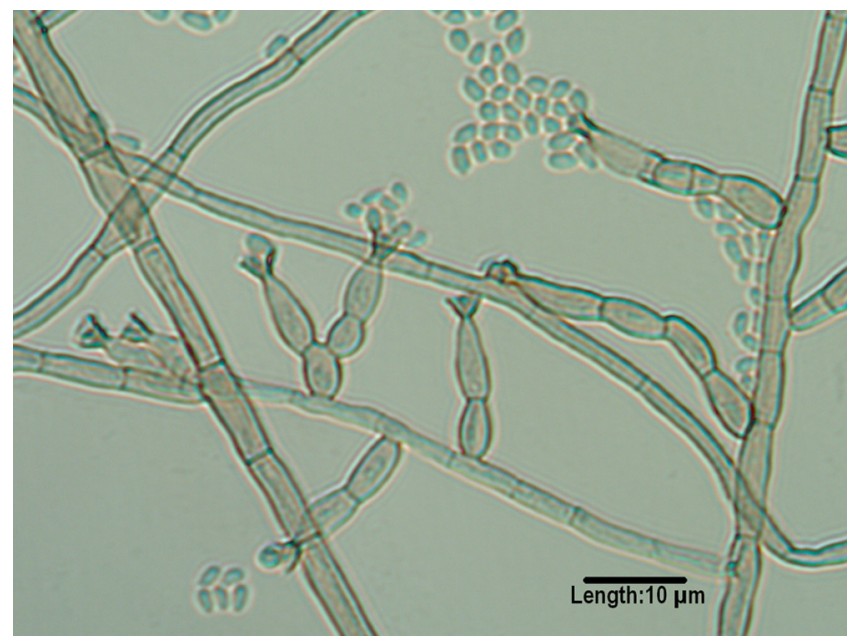

FIG. 10. Dark, funnel-shaped collarettes at the tips of the conidiogenous cells (phialides) in Phialophora verrucosa. Also note the ovalshaped conidia. 


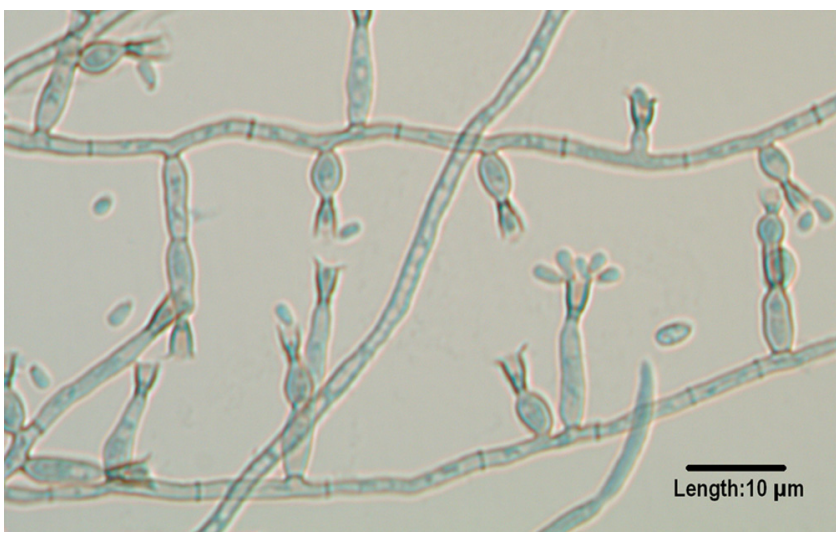

FIG. 11. Deep, dark, vase-shaped collarettes in Phialophora americana.

Veronaea. Initial reports of infection due to Veronaea botryosa were clustered in China; however, a more global distribution is now recognized, with cases seen in Libya, Philippines, an island in the Indian Ocean, and the United States. Two cases are noteworthy as agents of subcutaneous disease in heart (725) and liver (251) transplant recipients. The genus has recently been reexamined at the molecular level by Arzanlou et al. (43).

\section{Microascales}

Scedosporium. The genus Scedosporium and its associated teleomorph Pseudallescheria were extensively reviewed by Cortez et al. in 2008 (153); therefore, the information provided here will augment that previously published and/or highlight new taxonomy, distribution, and disease. Scedosporium prolificans, which is closely related but not a member of this complex, appears to occupy a more restricted geographic range, with infections occurring mainly in Australia, Spain, and the United States (747). Clinical discussion (see Clinical Syndromes and Their Management below) will be limited to $S$. prolificans, as related species have been extensively reviewed elsewhere (153) and may not reveal phaeoid hyphae in tissue, in contrast to the case for $S$. prolificans. Infection with this organism is of major concern in all settings due to its refractoriness to antifungal therapy and associated high mortality $(18,29,76,96,119,153$, $312,475,538,664,802,806)$. A recent review of 162 cases reported in the literature summarizes major risk factors as malignancy (46\%), cystic fibrosis $(12 \%)$, and solid organ transplantation $(9 \%)$ and chief clinical presentations as disseminated infection $(44 \%)$ and pulmonary mycoses $(29 \%)$, followed by bone and joint infections (10\%) (638). All disseminated infections were in individuals with underlying disease, primarily hematological malignancies; $70 \%$ of these had positive blood cultures, and mortality in this group was $88 \%$. Molecular characterization by ITS, D1/D2, translation elongation factor $1 \alpha$, and the chitin synthase genes for 20 cases of $S$. prolificans infection occurring in Germany between 1993 and 2007 suggests the possibility of two or three distinct genotypes (747). This finding may further our understanding of the epidemiology of this organism. Multiple genotypes were previously suggested by inter-simple-sequence-repeat (ISSR) fin- gerprinting (708). Increased numbers of infections with $S$. prolificans have also been reported from France (304) and Australia (190). Inflated annellides, a key microscopic feature in the identification of this organism, may be subtle in some isolates and easily overlooked. However, the colony color of $S$. prolificans is always darker than for other Scedosporium species.

Scopulariopsis. The genus Scopulariopsis is unusual in containing both hyaline and dark species. Most pigmented species associated with disease are anamorphs of various Microascus species detailed in Teleomorphic Genera below. Scopulariopsis shares an annellidic method of conidiogenesis with Scedosporium species but can be differentiated from this genus by conidial formation in chains rather than in clumps.

\section{Sordariales}

Madurella. An agent of dark grain mycetoma primarily in West Africa, M. mycetomatis has recently been proven to be a member of the Sordariales (172), unlike M. grisea, which resides in the Pleosporales. Isolates are very slow growing, produce a brown diffusible pigment, grow at $40^{\circ} \mathrm{C}$, and frequently remain sterile in culture; however, lateral phialides and globose conidia are occasionally produced. Precise identification is facilitated by DNA sequencing. Molecular characterization of 38 different $M$. mycetomatis isolates from Sudan has shown them to have identical DNA patterns, suggesting that host susceptibility rather than differential virulence is the determining factor in clinical presentations (10).

Myceliophthora. Myceliophthora thermophila is a thermophilic fungus common in high-temperature areas such as compost and exhibits growth at $50^{\circ} \mathrm{C}$. Colonies are pale brown, and conidia are borne from ampulliform swellings. Reports suggest that its recovery from tissue, even with a heavy fungal burden, may be difficult (196). The organism is also uncommonly seen in the laboratory and may provide identification challenges. It has been fatal in a disseminated case (95) and in a patient with aortic involvement with medial necrosis (234). A severe case of osteomyelitis was also reported following extensive injury to a knee and distal femur following a barnyard pitchfork injury (196).

Acrophialophora. Acrophialophora fusispora is an uncommonly seen agent occasionally microscopically misidentified as Scedosporium prolificans. The two species have similarly inflated conidiogenous cells, although they are phialidic versus annellidic and conidia are produced in chains rather than clusters in $A$. fusispora and $S$. prolificans, respectively. The organism grows at $40^{\circ} \mathrm{C}$, colonies display a striking darkening centrally (both front and reverse), and it produces finely echinulate conidia demonstrating various degrees of spiral banding. It has been reported as an agent of cerebral phaeohyphomycosis in a leukemic child (24), as an agent of keratitis (691), and as an agent of keratouveitis in association with a retained intraocular lens (41).

\section{Calosphaeriales}

Phialemonium. The genus Phialemonium was initially described to accommodate organisms closely resembling Acremonium spp. but containing pigmentation, although colonies of- 


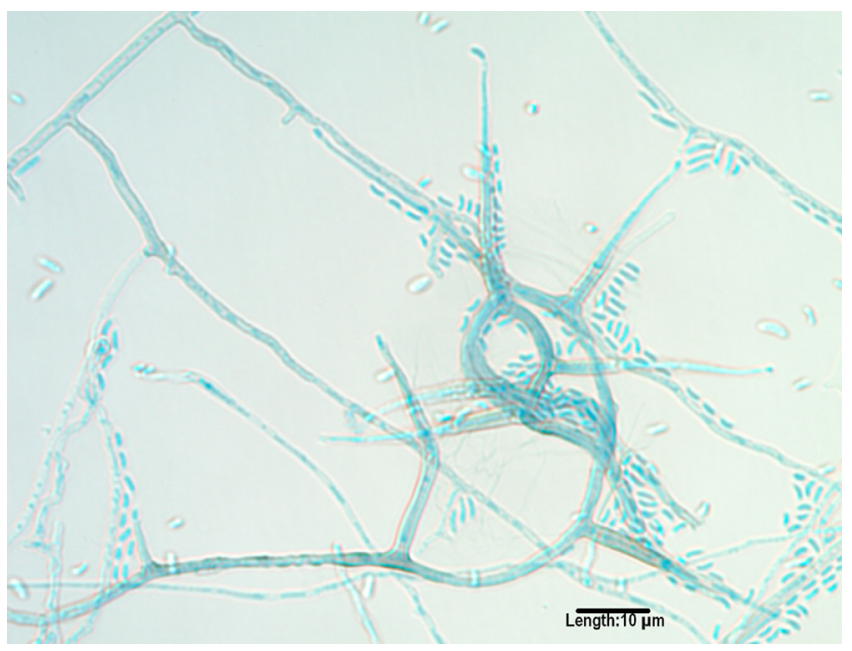

FIG. 12. Melanized hyphae, demonstrating warts (bottom), long robust phialides, and allantoid (curved) conidia of Phaeoacremonium parasiticum.

ten remain pale (264). Colonies are typically moist to slightly filamentous, and conidiogenous cells are a mixture of mediumlength phialides and adelophialides (short phialides lacking a basal septum). The genus currently contains two species of clinical interest, $P$. obovatum and $P$. curvatum. $P$. obovatum produces a green, diffusible pigment; has obovate conidia (like an upside-down egg); and has been reported as an agent of fatal endocarditis in a neonate (273). P . curvatum isolates range from cream to yellowish to pale brownish and have allantoid (curved) conidia. Infections attributed to $P$. curvatum include cutaneous and subcutaneous disease, disseminated infection, endophthalmitis, peritonitis, arthritis, and fungemia $(167,264,308,793)$. Also reported are cases of hemodialysisassociated endovascular infections (608) and endocarditis (561), with some cases linked to intracavernous penile injections in men frequenting impotence clinics (717). Several recent cases have demonstrated sporodochial formation in $P$. curvatum, a feature not previously seen in this species (167, 608, 793). Rivero et al. have recently reviewed published Phialemonium cases (634).

Phaeoacremonium. The genus Phaeoacremonium initially accommodated isolates with features similar to those seen in both Acremonium and Phialophora (159). It differs from the former by having pigmented hyphae and conidiophores and from the latter by having indistinct collarettes and warty conidiogenous cells. A recent morphologic and molecular characterization of the genus using $\beta$-tubulin sequences (525) has more clearly defined the genus and provided differential features for clinically significant species. Human pathogens include $P$. parasiticum (obsolete Phialophora parasitica) (Fig. 12) (335), P. alvesii (567), P. amstelodamense, $P$. griseorubrum, $P$. krajdenii (525), $P$. rubrigenum (491), $P$. tardicrescens, and $P$. venezuelense $(309,525)$. Infections caused by $P$. parasiticum include subcutaneous abscesses (245), thorn-induced arthritis (651), and disseminated infection (54). Colony colors may range from yellowish brown to orange-brown to brown to lavender.
Pleurostomophora. Clinically significant species in the mostly wood-inhabiting genus Pleurostomophora include $P$. richardsiae (obsolete Phialophora richardsiae) and P. repens (obsolete Phialophora repens), and individuals acquiring these mycoses are commonly immunocompromised $(369,601,815)$. Species are anamorphs of the genus Pleurostoma. P. richardsiae is characterized microscopically by distinctive flaring collarettes (Fig. 1) and both globose and oval conidia. The colonies of both species tend to be brown rather than gray or olivaceous. Human infections include subcutaneous cases (311) and bone disease (761).

\section{Coniochaetales}

Lecythophora. Two Lecythophora species, L. mutabilis and L. hoffmannii, are of clinical significance. Both produce orange, moist colonies initially, with central darkening in L. mutabilis as pigmented chlamydospores are produced. Organisms are agents of endophthalmitis (677), sinusitis (485), and prosthetic valve endocarditis (207). Recent large-subunit rDNA sequencing confirms the association of Lecythophora species with teleomorphs in the genus Coniochaeta (792) in the order Coniochaetales (361).

\section{Ophiostomatales}

Sporothrix. Sporotrichosis occurs worldwide, with the primary agent of disease being Sporothrix schenckii. The disease is commonly acquired by implantation of the fungus from various types of woody/plant material. Lymphocutaneous lesions are the norm; however, pulmonary disease and disseminated infections may occur in patients with underlying diseases (177). As a dimorphic fungus, it exhibits cigar-shaped yeasts in tissue and at $35^{\circ} \mathrm{C}$ and filamentous growth in culture. Only the sessile conidia borne along the sides of the hyphae are melanized. In a recent study characterizing the genus by calmodulin sequencing (480) and critically reviewing morphologic/physiologic features, these sessile conidia were shown to vary according to species within the $S$. schenckii species complex (479). They are elongate to triangular in $S$. schenckii and globose to subglobose in S. brasiliensis and S. globosa.

\section{ANAMORPHIC COELOMYCETE GENERA}

\section{Pleosporales}

Phoma and Phoma-like pycnidial coelomycetes. Several genera of morphologically similar pycnidial coelomycetes are occasionally recovered in cases of human subcutaneous disease (307, 585, 704), endophthalmitis (685), and deep tissue infection (411); however, their documentation and reporting as etiologic agents is limited by a lack of adequate identification (727). They include Phoma, Pleurophoma, Pleurophomopsis, and Pyrenochaeta species, with small, hyaline, typically onecelled conidia, and Coniothyrium (411, 704), Paraconiothyrium (773), and Microsphaeropsis species (307, 585, 685), with pale brown to dark, one-celled conidia (Fig. 13). The morphologic features of species within several sections in the genus Phoma have been detailed by Boerema et al. (84). Species in these similar genera are best differentiated by ITS sequencing. 


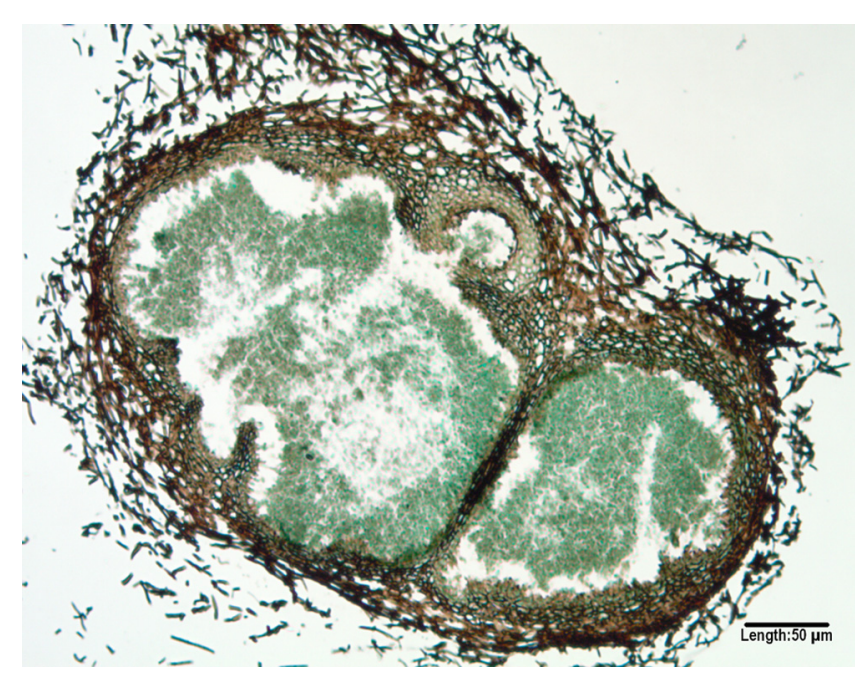

FIG. 13. A GMS-stained cross section of a multilocular pycnidium of a Microsphaeropsis species produced on carnation leaf agar after 5 weeks of incubation at $25^{\circ} \mathrm{C}$.

\section{Botryosphaeriales}

Lasiodiplodia. L. theobromae is a pycnidial coelomycetous organism incriminated in cases of subcutaneous disease (720), pneumonia in a liver transplant recipient (805), and ocular infections $(615,705)$. Conidia may take several weeks to mature and are distinctive, large (20 to 30 by 10 to $15 \mu \mathrm{m}$ ), and hyaline and single celled initially, becoming dark, striated, and two celled at maturity. The organism was formerly known as Botryodiplodia theobromae.

Macrophomina. Macrophomina phaseolina has been recently reported as an agent of disseminated disease in a renal transplant recipient (735) and as an agent of a cutaneous infection in a child with acute myeloid leukemia (714). The species is difficult to identify without sequencing, as the isolate typically remains sterile in culture, producing only sclerotia (sterile hard masses of hyphal elements).

Neoscytalidium. Neoscytalidium dimidiatum, previously known as Scytalidium dimidiatum $(518,519)$ is a rapidly growing, black, woolly, arthroconidia-producing mold. Microscopically similar hyaline variants lacking melanin, formerly referred to as Scytalidium hyalinum (639), should also be referred to as $N$. dimidiatum. The species may also produce a coelomycetous pycnidial synanmorph with extended incubation on appropriate media. The name Nattrassia mangiferae has now been placed in the new genus Neofusicoccum $(158,735)$. This organism is a plant pathogen, and the name should not be used for human isolates. $N$. dimidiatum primarily produces infections mimicking those caused by dermatophytes on skin and nails $(218,459)$, although there are occasional reports of ocular infections (26) and deep mycoses in immunocompromised individuals $(73,476,694,801)$.

\section{Sordariales}

Phomopsis. There are only rare reports of Phomopsis species in human disease. Similarly to Phoma and related genera, they are rarely identified beyond the genus level. They are recog- nized by their black, pycnidial conidiomata (globose to subglobose structures lined with conidiogenous cells) that produce hyaline alpha (ellipsoidal) and beta (long, filamentous, curved) conidia. One report concerns a case of osteomyelitis of the finger in a diabetic patient (727).

\section{TELEOMORPHIC GENERA}

\section{Sordariales}

Chaetomium and Achaetomium. Two ascomycetous genera known to produce their sexual state in culture are Achaetomium and Chaetomium. The fruiting body in both is a perithecium (a flask-shaped ascoma with an apical opening). Rarely are conidia produced. Species are differentiated mostly phenotypically by the size and shape of ascomata and the type of setae they possess, the size and shape of their brownish ascospores, and temperature tolerance. Most species fail to grow at $35^{\circ} \mathrm{C}$ and above and are common degraders of various organic compounds. The human pathogen C. globosum grows at $35^{\circ} \mathrm{C}$ but not $40^{\circ} \mathrm{C}$, and reports of invasive disease due to this and other, unidentified species $(34,449,742,814)$ are inadequately documented and most likely due to neurotropic species. Chaetomium atrobrunneum (314) and C. perlucidum do grow at $40^{\circ} \mathrm{C}$, are neurotropic (64), and should be considered in the differential diagnosis of CNS fungal disease. A key for identification of clinically significant species has been published by Barron et al. (64). The closely related Achaetomium strumarium is pale in culture and produces a reddish-purple diffusible pigment, ascospores similar to those of pathogenic Chaetomium spp., and occasional lateral, sessile conidia. It is also neurotropic and an agent of CNS phaeohyphomycosis with growth at $40^{\circ} \mathrm{C}(1,40)$.

\section{Pleosporales}

Leptosphaeria. Leptosphaeria senegalensis and the related $L$. tompkinsii are agents of black grain mycetoma mostly restricted to northern West Africa and India (177). In culture, colonies are slow growing and woolly, and black closed ascomata (cleistothecia) are immersed in the agar. Maturation of ascomata and ascospores is facilitated on plant-based media, and species are differentiated by ascospore features $(216,217)$.

\section{Microascales}

Microascus. Several pigmented Scopulariopsis species go on to produce their Microascus perithecial teleomorphs in culture. Several of these species have been documented as agents of fatal disease, particularly in transplant recipients. $M$. cinereus caused a brain abscess in a bone marrow transplant recipient (53), suppurative cutaneous granulomata in a patient with chronic granulomatous disease (483), and endocarditis of a prosthetic valve (129). M. cirrosus was the etiologic agent of disseminated disease in a pediatric bone marrow recipient (424), and M. trigonosporus was reported in a fatal pneumonia in another bone marrow transplant recipient (517). Microascus species are differentiated primarily by the size/shape of the perithecia, the length of the perithecial necks (Fig. 14), and the size and shape of the reddish-brown ascospores, which are 


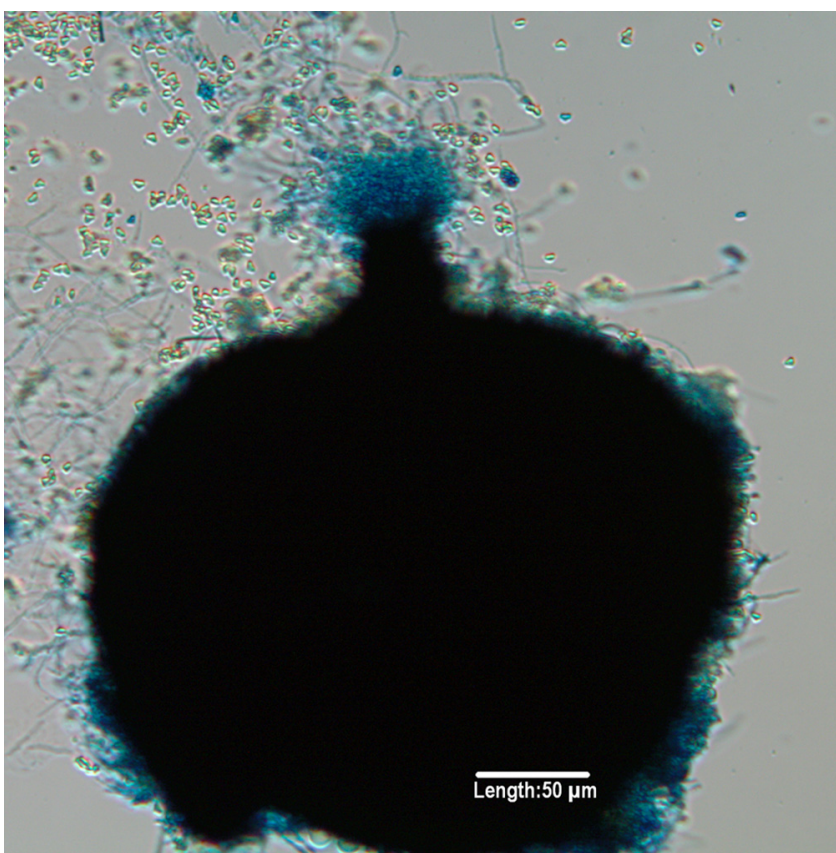

FIG. 14. Perithecium of Microascus trigonosporus formed on potato flake agar after 3 weeks of incubation at $25^{\circ} \mathrm{C}$. Note ascospores being released from the ostiole in the neck of the perithecium.

orange section shaped in $M$. cinereus, heart shaped in $M$. cirrosus (Fig. 15), and triangular in M. trigonosporus (Fig. 16).

Pseudallescheria. As discussed above for the anamorphic genus Scedosporium, Cortez et al. extensively reviewed Pseudallescheria/Scedosporium in 2008 (153), and so only subsequent taxonomic changes will be discussed here. The human pathogenic species as defined by recent molecular studies are as follows: Pseudallescheria boydii (anamorph Scedosporium boydii), Pseudallescheria apiosperma (anamorph Scedosporium apiospermum, heterothallic, not forming its teleomorph in culture, and D-ribose negative), and Pseudallescheria ellipsoidea (281-283). Other species of clinical interest in the P. boydii species complex include $S$. aurantiacum $(190,281)$ and $S$. dehoogii (282).

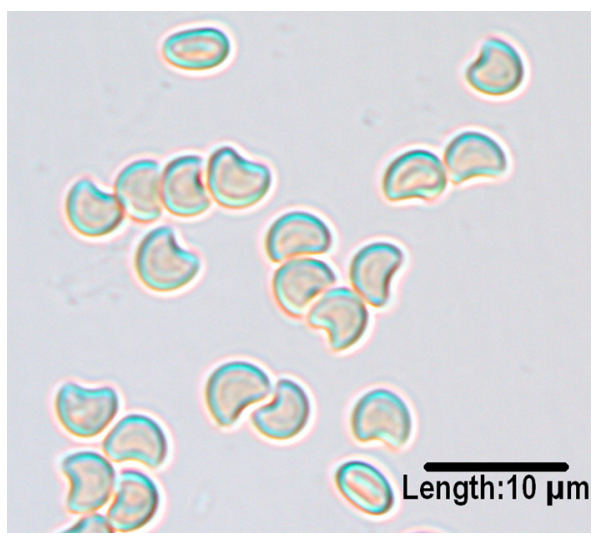

FIG. 15. Heart-shaped ascospores of Microascus cirrosus produced on potato flake agar after 3 weeks of incubation at $25^{\circ} \mathrm{C}$.

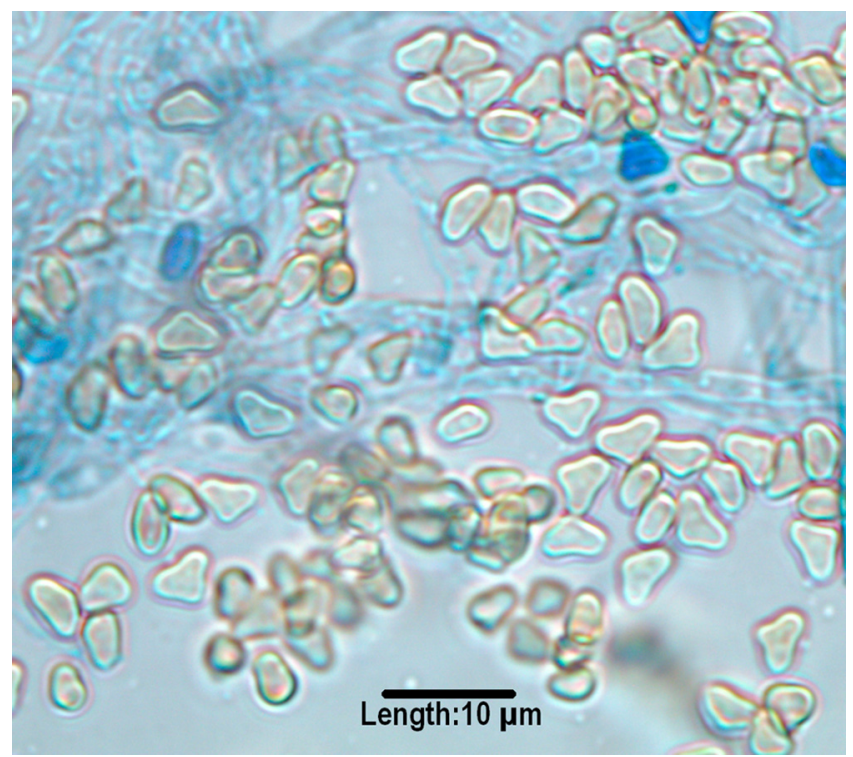

FIG. 16. Triangular ascospores of Microascus trigonosporus produced on potato flake agar after 3 weeks of incubation at $25^{\circ} \mathrm{C}$.

\section{PATHOGENESIS}

Surveys of outdoor air for fungal spores routinely show dematiaceous fungi (687). This suggests that all individuals are exposed, though few develop disease. Exposure is primarily from inhalation or minor trauma, which is frequently not even noticed by the patient. Relatively little is known regarding the pathogenic mechanisms by which melanized fungi cause disease, particularly in immunocompetent individuals.

\section{Role of Melanin}

One of the likely virulence factors is the presence of melanin in the cell wall, which is common to all dematiaceous fungi, though relatively few species have been studied (439, 522, 573, 666). Melanin in fungi is derived primarily from either dihydroxyphenylalanine (L-DOPA) or dihydroxynaphthalene (DHN) (437). Dematiaceous fungi contain only DHN melanin; L-DOPA melanin has not been described to our knowledge $(122,274,439,766)$. It is generally localized to the cell wall, though the exact mechanism of its production is poorly understood. In the species $F$. pedrosoi, melanin is produced in melanosomes associated with $\mathrm{Fe}^{2+}$ and $\mathrm{Ca}^{2+}$ and then transported to the cell wall (253). Melanin is extremely resistant to a variety of physicochemical agents, including free radical compounds, toxic metals, desiccation, and even ionizing radiation $(165,249$, 331, 795). A species of Chaetomium was isolated from grass that had been frozen in a glacier for over 5,000 years (331).

Considerable work has been done to elucidate the virulence potentials of several fungi (dematiaceous and nondematiaceous) that contain melanin, notably Aspergillus fumigatus, Cryptococcus neoformans, E. dermatiditis, and S. prolificans (123, 134, 201, 203, 205, 284, 428, 549, 573, 653, 671, 730, 756). There are multiple proposed mechanisms by which melanin may act as a virulence factor $(109,324,375)$. It may confer a protective advantage by scavenging free radicals and hypochlorite that are produced by phagocytic cells in their oxidative 
burst and that would normally kill most organisms $(376,671)$. In addition, melanin may bind to hydrolytic enzymes, thereby preventing their action on the plasma membrane, and to antifungal drugs, preventing their action (370, 375, 547, 768). There is also evidence that certain melanized fungi are less susceptible to phagocytosis and killing by neutrophils and macrophages $(334,584)$. These multiple functions may help explain the pathogenic potential of some dematiaceous fungi, even in immunocompetent hosts. Specifically, in E. dermatitidis, disruption of melanin production leads to markedly reduced virulence in animal models and restriction of hyphal growth (103, 201, 203, 428). However, hyphae of $S$. prolificans were found to be more susceptible to damage from neutrophils than $A$. fumigatus (284). Melanin has also been shown to reduce the susceptibility of $M$. mycetomatis to ketoconazole and itraconazole by binding these drugs (766). Though only a minority of dematiaceous fungi have been studied, it is likely that melanin plays a critical role in pathogenesis for clinically important species.

\section{Other Putative Virulence Factors}

It is interesting to note that most allergic disease and eosinophilia is caused by three genera, Alternaria, Bipolaris, and Curvularia (622). The virulence factors in these fungi that are responsible for eliciting allergic reactions are not well understood, though Alternaria was found to stimulate the degranulation of eosinophils, possibly due to an aspartic protease $(372,496)$. These organisms are very common in the environment, so exposure is practically universal, though the incidence of allergic disease is relatively low, suggesting that host factors may play a role. A study by Schubert et al. found that HLA-DQB1*03 was associated with allergic fungal sinusitis (676). Further studies are needed to better delineate the importance of virulence factors other than melanin.

\section{DIAGNOSIS}

The timely and accurate diagnosis of fungal infections by melanized fungi consists of a multifaceted approach. With the exponential increase in immunocompromised individuals, particularly those in tertiary care cancer centers (72), this becomes imperative to prevent potentially fatal outcomes. Standard conventional diagnostic procedures include direct microscopy, histopathological stains to document tissue invasion, radiographic and computerized tomography (CT) findings, and isolation procedures to recover the fungus and identify the etiologic agent. The clinical presentation and diagnostic findings segregate these infections into the major categories of eumycetoma, chromoblastomycosis, and phaeohyphomycosis. Phaeohyphomycosis maybe further delineated depending upon whether infections are superficial or deep, by their anatomic location, and by the host's response. The microscopic features seen in phaeohyphomycosis, however, are similar regardless of the anatomic site. The confusion surrounding the placement of members of the Sporothrix schenckii and Pseudallescheria boydii species complexes within the dark molds is related to their tissue presentation as yeast cells and hyaline hyphae, respectively. As the term phaeohyphomycosis is commonly used to describe fungi with dark hyphae in tissue, these organisms

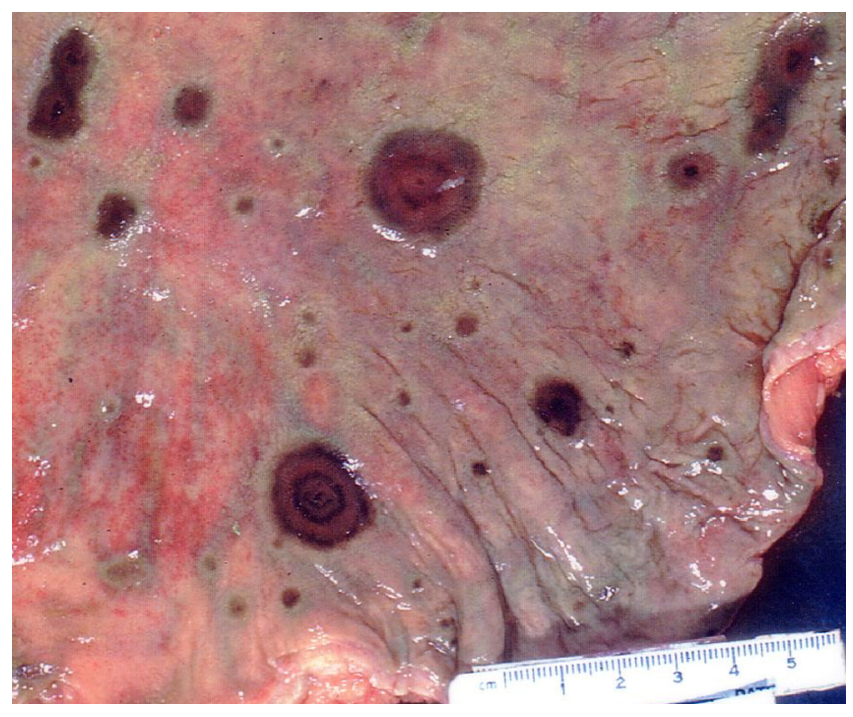

FIG. 17. Bipolaris spicifera colonies in stomach mucosa of patient with disseminated disease (autopsy). (Reproduced from reference 624 [original Fig. 15-6A] with kind permission of Springer Science and Business Media.)

would be excluded; however, both produce melanized conidia in culture. As a thorough review of infections caused by Pseudallescheria/Scedosporium species has recently been published (153), this paper will concentrate on taxonomic changes and new species documented as etiologic agents subsequent to that review.

\section{Initial Specimen Processing}

The appropriate specimen collection, transport, and processing procedures are important considerations in the demonstration of melanized fungi in tissue and their recovery in culture. The most useful diagnostic specimens are those collected at the source of infection; however, specimens peripheral to the site of infection, such as blood cultures in hematogenously disseminated disease, may also be diagnostic in the absence of focal manifestations or when foci are not easily accessible. Appropriate specimens for the recovery of fungi are detailed elsewhere in several reference works (723). Specimens commonly obtained for recovery of melanized fungi include tissue biopsy specimens, aspirates, and body fluids. Surgically obtained specimens should always be cultured as well as processed for histopathology, and the inoculum should be finely sliced or minced rather than ground (as in the case for recovery of $H$. capsulatum). Gross examination may occasionally reveal evidence of melanized fungi as well (Fig. 17). Small volumes of sterile body fluids may also be concentrated by syringe filtration $(0.2 \mu \mathrm{m})$. Several blood culture systems are available, and the maximum amount of blood recommended should always be used. Swab cultures from superficial sites are usually not representative of the disease process, frequently contain indigenous contaminating mycobiota, and should generally be avoided. Grains or granules should also be washed several times in antimicrobial-containing saline to avoid bacterial overgrowth (504). Also compromising etiologic agent recovery is a delay in specimen transport. Optimally, most specimens 


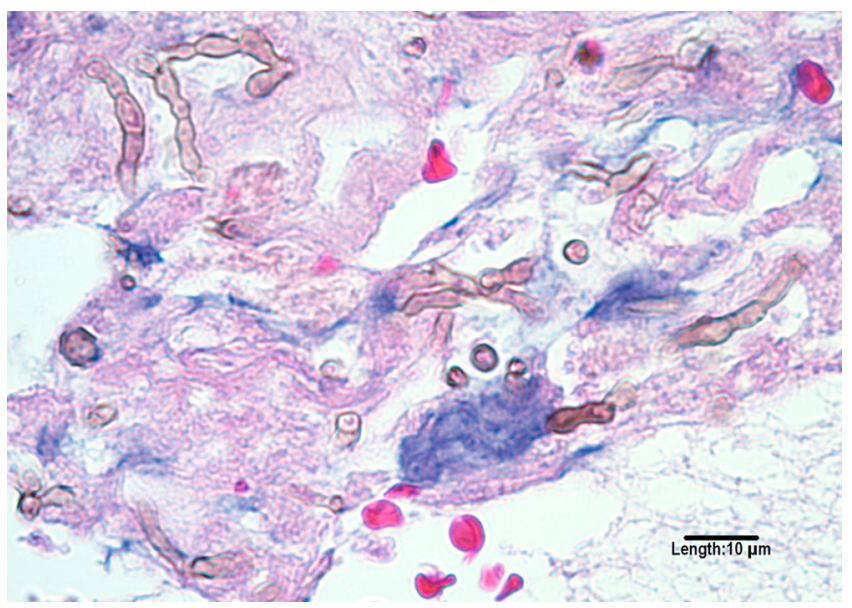

FIG. 18. H\&E stain of melanized, moniliform hyphal elements of Cladophialophora bantiana from a brain abscess.

should be processed within $2 \mathrm{~h}$ of collection, and cerebrospinal fluid should never be refrigerated (723).

Guidelines regarding the handling of potentially infectious fungi in the laboratory setting are available. It is suggested that cultures of certain well-known pathogenic fungi, such as $\mathrm{Coc}$ cidioides immitis/posadasii and $H$. capsulatum, be worked with in a biosafety level (BSL) 3 facility, which requires a separate negative-pressure room, though clinical samples may be handled under BSL 2 conditions (130). Recently, certain agents of phaeohyphomycosis, in particular C. bantiana, have been included in the list of fungi that should be kept under BSL 2 containment (130), and in Europe this mold is considered a hazard category 3 agent (one that can cause severe human disease) (231). This seems reasonable given its propensity, albeit rare, for causing life-threatening infection in healthy individuals.

\section{Direct Microscopy}

Due to the ubiquitous nature of melanized fungi, examination of direct specimens is critical, as the finding of fungal elements within tissue is required to document a black mold as the etiologic agent when recovered in culture. It should also be noted that in individuals receiving antifungal therapy, hyphae seen in tissue may be the only evidence of disease, as growth in culture may be severely suppressed or absent. Conversely, recovery in culture without visualization in tissue should be interpreted with caution. Isolation of the same organism multiple times or from multiple sites also supports its role in disease when microscopic evidence is lacking. Commonly used methods for the direct examination of specimens include the Gram stain, several different concentrations of $\mathrm{KOH}$ preparations (with or without the incorporation of mycological stains), and the fluorescent calcofluor white stain (652). The Gram stain and $\mathrm{KOH}$ preparations are rapid, easily performed tests that should not be overlooked when making an initial assessment of fungal disease with appropriate clinical specimens. Each is described in detail in various microbiology texts $(441,591)$. Calcofluor and related fluorochromes that bind to cellulose

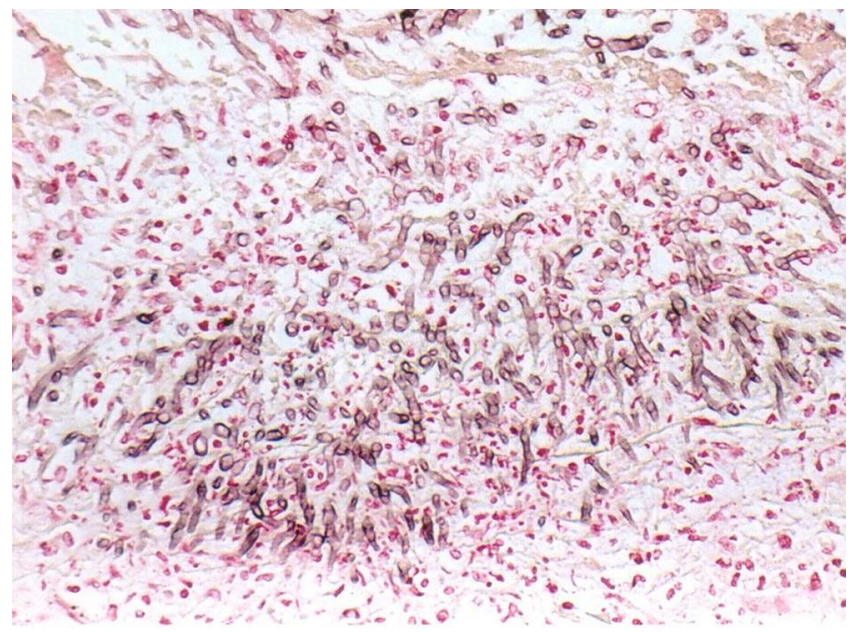

FIG. 19. Bipolaris spicifera in lung tissue (Fontana-Masson stain; magnification $\times 100$ ). (Reproduced from reference 624 [original Fig. 15-13B] with kind permission of Springer Science and Business Media.)

and chitin in fungal cell walls provide another rapid stain for demonstrating fungi by utilizing fluorescence (326). A fluorescence microscope with broadband excitation filters in the range of 300 to $412 \mathrm{~nm} \mathrm{(322)} \mathrm{and} \mathrm{eye} \mathrm{barriers} \mathrm{are} \mathrm{required}$ (441). Diagnostic structures seen by direct microscopy also vary according to the clinical presentation. In cases of eumycetoma incited by dark fungi, the demonstration and appearance of pigmented grains or granules (bundles of hyphae often embedded in a cement-like matrix) from pus, exudates, bandage gauze, and biopsied tissue are highly significant and narrow the potential etiologic agents to a limited number of black molds known to cause mycetoma. Members of the Pseudallescheria boydii species complex and Phaeoacremonium species, however, produce pale grains in tissue $(159,503)$. Fungi responsible for grains or granules expressed from draining sinus tracts are best visualized in permanent histopathological preparations, as are sclerotic bodies seen in chromoblastomycosis. Fungal elements seen in phaeohyphomycosis are frequently detected by direct microscopy; however, tissue invasion is best documented by permanent histopathological stains. The Gram stain may also be useful in some settings with fungi often demonstrating variable staining. Note that the hyphae are often Gram negative while the conidia are Gram positive; however, either structure may be Gram variable.

\section{Histopathology and Special Stains}

Several histopathological stains are useful for the demonstration of melanized fungi (670). The most frequently used hematoxylin-and-eosin (H\&E) stain demonstrates pigmentation in hyphae that are strongly melanized (Fig. 18). In fungi that are only lightly pigmented, hyphae may be misidentified as hyaline rather than dark. The melanin Fontana-Masson stain (Fig. 19) is useful in these situations to visualize the phaeoid nature of hyphae in tissue, though other molds may occasionally stain strongly as well (414). An additional stain useful for dark hyphae is the periodic acid-Schiff (PAS) stain (Fig. 20). It 


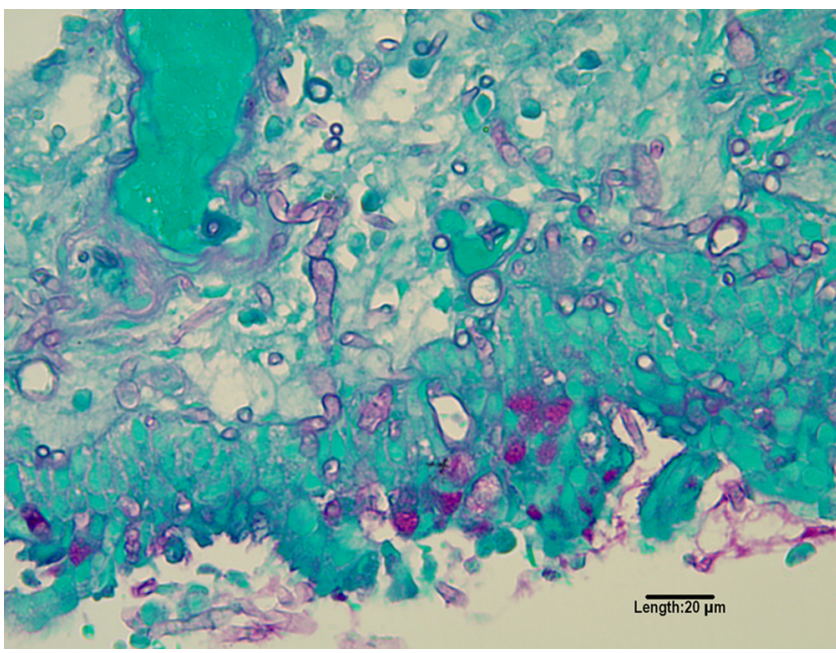

FIG. 20. PAS stain of Ochrocladosporium elatum, formerly Cladosporium elatum, from sinus tissue.

is frequently preferred over $\mathrm{H} \& \mathrm{E}$ due to the more vivid colors of hyphae, which stain a bright pink-purple against a green background; however, it may overshadow the melanin when present. For practically all fungal histopathology, a Gomori methenamine silver (GMS) stain is ordered. Its utility is in the dramatic visualization of hyphae as dark elements against a green background; however, it fails to discriminate between pigmented and nonpigmented fungal elements (Fig. 21). Note that in Fig. 19 and 21 many of the fungal elements are either short stubby hyphae, pseudohyphae, or moniform (bead-like) hyphae. This is not an uncommon tissue presentation with several dematiaceous genera and is quite different from that seen in aspergillosis, fusariosis, or zygomycosis. Chromoblastomycosis (Fig. 22) presents in tissue as brown, compact muriform hyphal elements with horizontal and vertical cross walls variously referred to as sclerotic bodies, Medlar bodies, or "copper pennies" (610).

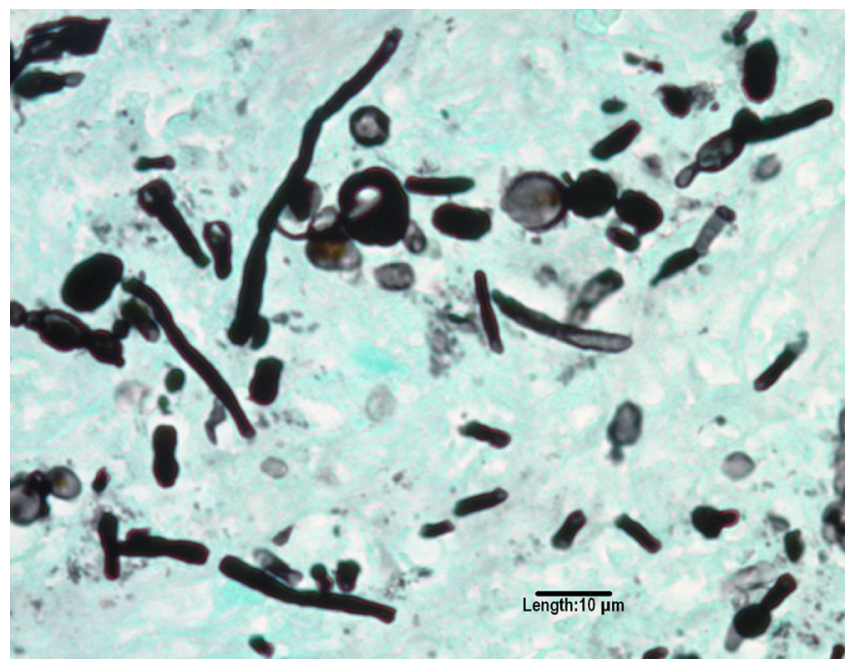

FIG. 21. GMS stain of Rhinocladiella mackenziei from a brain abscess. Note the many moniform hyphal elements often seen with melanized fungi.

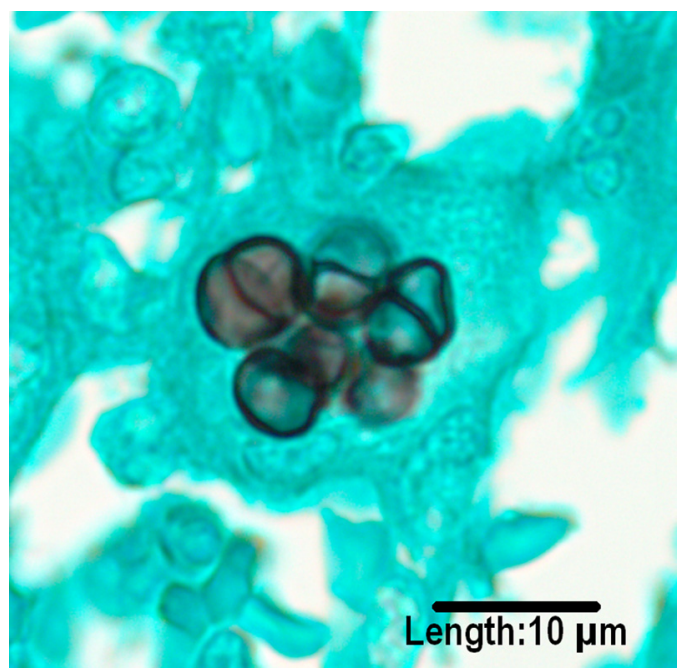

FIG. 22. GMS stain of sclerotic bodies produced by Fonsecaea pedrosoi.

\section{Isolation Procedures and Culture}

These ubiquitous fungi can be contaminants in cultures, making the determination of clinical significance problematic. A high degree of clinical suspicion as well as correlation with appropriate clinical findings is required when interpreting culture results. The recovery of dematiaceous fungi from clinical specimens requires the appropriate media and incubation conditions. Various references list suggested schemes for primary isolation media $(722,723)$. A common approach is to include nonselective as well as selective media, such as those containing cycloheximide, media enriched for fastidious organisms such as brain heart infusion agar (BHI) and inhibitory mold agar (IMA), and also media containing antimicrobial agents to suppress bacteria in specimens collected from nonsterile sites. A nonselective medium frequently employed is SDA. While growth is adequate, the color of the colonies is often cream to pale orange or light brown, making their recognition as phaeoid genera difficult. When these same isolates are transferred to plant-based media, they assume their more typical olivaceous to dark brown to black color. The additional time required for these subcultures can have potentially devastating consequences in the profoundly compromised individual, and therefore the use of a plant-based medium initially, such as PDA, is highly recommended. Cultures are commonly incubated at $30^{\circ} \mathrm{C}$; however, room temperature at or near $25^{\circ} \mathrm{C}$ may also be used. Cultures should be examined every day for the first 3 days and twice a week thereafter. Most phaeoid molds are recovered within a week, and incubation of negative cultures beyond 3 weeks is seldom necessary (430). Substantially longer incubation, however, may be required for development of diagnostic structures in some isolates, particularly for coelomycetes or homothallic ascomycetes. All filamentous organisms should be manipulated and examined under a certified biological safety cabinet. 


\section{Radiology}

There are few radiologic features that distinguish melanized fungi from other molds as causes of infection. The "dot-incircle" sign has been noted to be specific for the finding of eumycetoma in magnetic resonance imaging (MRI) studies (141). This is felt to be due to the low signal intensity produced by the fungal grains in tissue.

\section{Antigen Testing and Serology}

There are no widely available serologic or antigen tests available to specifically detect melanized fungi in blood or tissue. However, serum antigen testing for 1,3- $\beta$-D-glucan (primarily for Candida spp.) and galactomannan (primarily for Aspergillus spp.) may cross-react with melanized fungi, though usually with low levels $(164,419)$. However, in immunocompromised patients with cultures positive for dematiaceous fungi, a positive serum galactomannan test may indicate concomitant infection with Aspergillus; careful clinical correlation is advised (71). Further studies are needed to better understand the nature and likelihood of cross-reactivity. Serum enzyme-linked immunosorbent assays (ELISAs) for $F$. pedrosoi and $C$. carrionii have been developed to aid in the diagnosis of chromoblastomycosis, though relatively low sensitivity and specificity have limited their usefulness, and only small numbers of patients have been studied $(552,775,778)$.

\section{Molecular Diagnostics}

In an effort to improve the rapidity with which invasive fungal infections are detected as well as the sensitivity and specificity of diagnostic tests, recent assays have focused on nonculture methods, in particular nucleic acid-based methods, such as PCR assays. Despite advances in the direct diagnosis of other, more common genera such as Candida, Aspergillus and Fusarium, the direct diagnosis of infections incited by melanized species remains a challenge. However, studies have begun to examine the potential of identifying species within this diverse group of fungi using PCR of highly conserved regions of ribosomal DNA (2). A panfungal PCR assay described by Lau et al. targeting the ITS1 region was able to detect several species of dark molds from fresh, formalin-fixed, or paraffinembedded tissue specimens, including $S$. prolificans, Exophiala spp., Exserohilum rostratum, and Microsphaeropsis arundinis (445). In a real-time PCR assay targeting part of the $28 \mathrm{~S}$ large-subunit rRNA gene, Vollmer et al. were able to amplify Aureobasidium pullulans in clinical specimens from intensivecare patients with either artificial respiration or infective endocarditis (785). While these methods and those to be refined in the foreseeable future will provide a more rapid diagnosis for some agents of phaeohyphomycosis, the diversity of black molds increasing in immunocompromised individuals makes their identification from direct materials a daunting task. Currently, a greater utility of molecular methods is in the identification, taxonomy, and phylogenetic placement of these melanized fungi.

\section{IN VITRO ANTIFUNGAL SUSCEPTIBILITY}

In vitro antifungal susceptibility testing has advanced considerably in the past several years, especially when one considers that a standardized method for testing yeasts was not available until 1997 (534), the first standardized method for filamentous fungi was not available until 2002, and both were updated in 2008 (147, 148). Due to the relatively recent development of antifungal susceptibility testing, the available in vitro data for dematiaceous fungi are relatively sparse, and often rely on small numbers of isolates per species. An important issue is that much of the older literature is often inconsistent with regard to methodology, making reliable observations difficult. In addition, as defined interpretive breakpoints are not available for any of the molds, guidelines for interpreting in vitro data frequently rely on close approximations to breakpoints for Candida species, as well as achievable concentrations of the drug using standard dosing regimens. A MIC of $\leq 1 \mu \mathrm{g} / \mathrm{ml}$ is often used as an indicator of potential susceptibility for most drugs used to treat black molds, excluding flucytosine (5-FC) $(<50 \mu \mathrm{g} / \mathrm{ml})$, recognizing that there are significant differences in pharmacological properties between the various agents as well as differences in drug concentrations tested. Lower MICs typically suggest better activity. The in vitro activities of several antifungal agents against a variety of dematiaceous fungi are presented in Table 3 . The data are from a compilation of the current literature $(49,50,161-163,224-228,250,266,294,315$, 382-384, 391, 532, 553, 625, 781, 782); however, clinical correlates are not available. Antifungal susceptibility testing of etiologic agents, when warranted, may assist in appropriate patient management.

\section{Polyenes}

Amphotericin B. Amphotericin B generally has good in vitro activity against most clinically important dematiaceous fungi. However, some species have been consistently resistant (MICs of $\geq 2 \mu \mathrm{g} / \mathrm{ml}$ ) in vitro, including $S$. prolificans and S. brumptii (507), while other species have occasionally been found to be resistant, including Curvularia spp., Exophiala spp., and $R$. mackenziei $(507,726)$. Significant toxicity often limits use of the standard formulation, primarily due to renal insufficiency, electrolyte disturbances, and infusionrelated side effects. However, nephrotoxicity has been significantly reduced by the development of lipid-associated formulations (339). Use of these preparations allows for much higher doses than possible with standard amphotericin B, which may improve their efficacy against these fungi. In addition, lipid amphotericin B preparations may achieve higher concentrations in brain as well (407).

Natamycin. Natamycin is a polyene antifungal used exclusively as topical therapy in eye infections, particularly keratitis. It has broad spectrum of activity against most relevant molds (Aspergillus and Fusarium) and is available in concentrations of up to $5 \%$, which is generally well tolerated $(478,650)$. Data for susceptibility against common dematiaceous fungi are very limited, though one study did show activity against Curvularia (800). 
TABLE 3. In vitro activities of antifungal agents against selected melanized fungi ${ }^{a}$

\begin{tabular}{|c|c|c|c|c|c|c|c|c|c|c|c|c|}
\hline \multirow{2}{*}{ Species } & \multicolumn{12}{|c|}{ Activity $^{b}$} \\
\hline & $\mathrm{AmB}$ & Itra & Vori & Posa & Isavu & Ravu & Keto & Terb & $5-\mathrm{FC}$ & Casp & Mica & Anid \\
\hline Alternaria spp. & + & ++ & + & ++ & + & & & + & - & + & & + \\
\hline Aureobasidium pullulans & + & ++ & ++ & & & & & & & & & \\
\hline Bipolaris spp. & + & ++ & ++ & ++ & + & + & & + & & + & & + \\
\hline Chaetomium spp. & + & ++ & ++ & & & ++ & + & & - & & & \\
\hline Cladosporium spp. & + & ++ & ++ & & & & & ++ & + & & + & \\
\hline Cladophialophora bantiana & ++ & ++ & ++ & ++ & & & + & + & + & + & ++ & + \\
\hline Curvularia spp. & + & ++ & ++ & ++ & + & + & & + & & + & & \\
\hline Exophiala spp. & + & ++ & ++ & ++ & + & + & & + & + & + & & + \\
\hline Exophiala dermatitidis & ++ & ++ & ++ & ++ & & + & + & + & + & & + & + \\
\hline Exserohilum spp. & ++ & ++ & ++ & & & + & & + & & & & \\
\hline $\begin{array}{l}\text { Fonsecaea pedrosoi } \\
\text { Lasiodiplodia theobromae }\end{array}$ & ++ & ++ & ++ & & & + & ++ & $\begin{array}{l}++ \\
+\end{array}$ & + & + & + & + \\
\hline $\begin{array}{l}\text { Lasiodiplodia theobromae } \\
\text { Madurella mycetomatis }\end{array}$ & + & ++ & ++ & & & & ++ & + & - & & & + \\
\hline Ochroconis gallopava & + & ++ & + & & & & +7 & + & - & & & + \\
\hline Phialemonium spp. & + & + & + & & & & & & & & & \\
\hline Phialophora spp. & + & ++ & & ++ & & + & & + & + & + & & + \\
\hline Rhinocladiella spp. & + & ++ & ++ & & & + & & & + & & & \\
\hline Rhinocladiella mackenziei & + & + & + & + & + & & & & + & - & & - \\
\hline Scedosporium/Pseudallescheria spp. & - & + & ++ & + & & & & & & & & \\
\hline Scedosporium prolificans & - & - & - & - & & & & - & & - & - & - \\
\hline Scopulariopsis brumptii & - & - & & - & & & & & & & & - \\
\hline Veronaea botryosa & & + & + & & & & & & & & & \\
\hline Wallemia sebi & + & + & + & + & & & & + & - & & + & \\
\hline
\end{tabular}

${ }^{a}$ Adapted from reference 625 with permission of Expert Reviews Ltd.

${ }^{b}$ Abbreviations: AmB, amphotericin B; 5-FC, flucytosine; Keto, ketoconazole; Itra, itraconazole; Vori, voriconazole; Posa, posaconazole; Ravu, ravuconazole; Casp, caspofungin; Mica, micafungin; Anid, anidulafungin; Terb, terbinafine; Isavu, isavuconazole. ++, good activity suggested based on consistently low MICs and testing against at least five isolates of a particular genus or species; +, potential/marginal activity suggested based on inconsistent MICs or very few isolates of a particular genus or species; -, no significant activity suggested based on consistently high MICs. The results do not represent formally defined CLSI breakpoints.

\section{Azoles}

The triazole agents itraconazole, voriconazole, and posaconazole demonstrate the most consistent in vitro activity against dematiaceous fungi, except against $S$. prolificans and $S$. brumptii, which are resistant to all azoles $(120,227,507,509)$. Only voriconazole is available as an intravenous (i.v.) formulation. All of these agents have significant drug interactions that must be considered during therapy (310). In addition, therapeutic drug monitoring is becoming increasingly utilized as data correlating serum levels with clinical response and toxicity accumulate (706).

Other azoles have a limited role in the therapy of these infections. Ketoconazole was the first oral azole and has a relatively broad spectrum. However, a number of side effects have significantly limited its current use with the availability of newer agents that are much better tolerated. Sparse in vitro data are available for dematiaceous fungi, but good activity is noted for the most common fungi causing chromoblastomycosis and mycetoma $(35,767)$. Fluconazole has negligible activity against dematiaceous molds $(132,259)$ and essentially no role in therapy given the variety of other options available, though anecdotal success has been reported (198).

Itraconazole. Though itraconazole was the first oral azole with significant activity against dematiaceous fungi and has had the most clinical use in therapy, concerns over adverse effects and the lack of an intravenous formulation have reduced its use in recent years. For itraconazole, the capsule form requires an acidic environment for absorption, while the suspension with cyclodextrin does not, being more consistently absorbed. Itraconazole demonstrates good activity against the vast ma- jority of dematiaceous fungi tested (226-228, 506, 507). MICs generally are $\leq 0.125 \mu \mathrm{g} / \mathrm{ml}$ for this group of fungi.

Voriconazole. Voriconazole has become the treatment of choice for invasive aspergillosis, supplanting amphotericin B for this indication $(385,745)$. It has also become a commonly used agent for treating many other invasive mold infections, especially those caused by dematiaceous fungi. The i.v. form is particularly useful in critically ill patients. It is generally well tolerated, though visual side effects are common but rarely limit therapy (745). In addition, like itraconazole, it has a broad spectrum of activity that includes most dematiaceous fungi $(227,228,507)$. However, MICs may be slightly higher for voriconazole, though the clinical significance of this is unclear.

Posaconazole. Posaconazole is the most recently released azole and has the broadest spectrum of any oral agent (338, $401,707)$. It is currently available only orally, though an i.v. formulation is being developed. It is generally very well tolerated. Oral absorption is significantly improved if it is administered with food, particularly food with a high fat content. The published in vitro data are relatively limited for dematiaceous fungi, but good activity is demonstrated against most species tested, including Bipolaris spp., C. bantiana, and R. mackenziei $(14,52,224,592)$. Posaconazole may be useful in cases of CNS disease, even that due to refractory molds (600).

Investigational azoles. Isavuconazole is a broad-spectrum azole with both oral and i.v. forms that has not been approved for use at this writing. Limited in vitro data exist for dematiaceous fungi $(294,532)$. Ravuconazole is another investigational azole with activity against a wide variety of molds (259). 


\section{Flucytosine}

Flucytosine (5-FC) is unique in its mechanism of action, inhibiting DNA and RNA synthesis $(252,774)$. In the United States it is available only in oral form. The development of resistance during monotherapy has resulted in its use in combination therapy for systemic mycoses, most notably cryptococcal meningitis (774). In vitro studies with dematiaceous fungi are limited, though activity against $C$. bantiana, Exophiala spp., and Fonsecaea $(F$. ) pedrosoi, the major etiologic agent of chromoblastomycosis, has been shown $(111,202)$.

\section{Allylamines}

Allylamines, like the azoles, also inhibit ergosterol synthesis, but they act on squalene epoxidase, an enzyme two synthetic steps before the target of azoles. Their clinical role has been limited to treatment of dermatophyte infections, though there has been recent interest in potentially expanding their clinical spectrum $(333,626)$. Terbinafine is the only oral allylamine available for systemic use. However, its extensive binding to serum proteins and distribution into skin and adipose tissue have diminished enthusiasm for its use in treating serious systemic fungal infections $(356,381,468,655)$. In vitro studies against dematiaceous fungi are emerging, and broad-spectrum activity has been seen, including against Alternaria, Curvularia, and Bipolaris and agents of chromoblastomycosis $(35,382$, 506). The in vitro testing range for this agent is typically between 0.004 and $2 \mu \mathrm{g} / \mathrm{ml}$.

\section{Echinocandins}

The echinocandins are the latest group of antifungal agents to be developed and have a unique mechanism of action, inhibiting 1,3- $\beta$-D-glucan synthesis and thereby disrupting the fungal cell wall (118). Caspofungin, micafungin, and anidulafungin are available only in an intravenous formulation and are generally well tolerated and, notably, have very few significant drug interactions (145). They are generally considered therapeutically equivalent, based on studies conducted on invasive Candida infections. In vitro studies with dematiaceous fungi are limited, with variable activity noted against many dematiaceous fungi, including Curvularia, Bipolaris, and $F$. pedrosoi (224, 225). S. prolificans appears to be resistant $(225,509)$. Micafungin may have lower MICs for $C$. bantiana than other echinocandins (225). In general, MICs for dematiaceous fungi are higher than those for Aspergillus spp.

\section{Other Agents}

A variety of drugs have been explored for activity against these fungi, given their refractory nature. Miltefosine, a drug originally developed as an anticancer drug and found to be effective in leishmaniasis, has antifungal activity against a variety of dematiaceous fungi, including $S$. prolificans (797). Nikkomycin, a chitin synthase inhibitor with activity against $\mathrm{Coc}$ cidioides immitis, was found to have relatively poor activity against dematiaceous fungi (454).

\section{Antifungal Combinations}

Uses of antifungal combinations are being increasingly studied as strategies for treatment of refractory fungal infections, though not extensively for dematiaceous fungi. However, for $C$. bantiana and $S$. prolificans, the most common fungi causing CNS and disseminated diseases, respectively, novel approaches are needed to improve therapy, and a variety of combinations have been studied in vitro and in vivo (see Animal Models of Infection below). In a murine model of $C$. bantiana, combination therapy was found to be superior to monotherapy for all agents tested (481).

Given that no single antifungal agent has significant activity against $S$. prolificans, numerous combinations have been studied to improve efficacy. The combination of itraconazole and terbinafine has been studied against $S$. prolificans, which is otherwise generally resistant to all agents. In vitro, synergistic activity against most isolates of this species was found, and no antagonism was noted (511). Voriconazole and terbinafine also display similar synergy in vitro (510). The mechanism is presumably potent inhibition of ergosterol synthesis at two different steps of the pathway by these agents. However, this should be interpreted with caution, as terbinafine is not recommended for systemic infections. The combination of voriconazole with micafungin and amphotericin B was found to be synergistic in vitro against $S$. prolificans, though double combinations also lowered individual MICs (637). Other reports suggest synergy against $S$. prolificans with voriconazole and caspofungin or with micafungin and amphotericin B (160, 716, 821). Importantly, antagonism was not observed with any of these combinations. Gil-Lamaignere et al. showed a synergistic effect of voriconazole or posaconazole with neutrophils in vitro against hyphae of $S$. prolificans (285). Older literature also suggests synergy with ketoconazole and 5-FC for a variety of dematiaceous fungi (152). This may be applicable to other azoles as well.

\section{ANIMAL MODELS OF INFECTION}

There are relatively few animal studies with dematiaceous fungi. One of the earliest studies was a murine model of infection with $E$. dermatitidis and $F$. pedrosoi (603). Amphotericin $\mathrm{B}$ and 5 -FC were active alone or in combination, though ketoconazole was not. In another study, 5-FC had the broadest activity against $C$. bantiana, $O$. gallopava, and $E$. dermatitidis in mice, followed by amphotericin B and fluconazole (despite resistance in vitro) (202). Terbinafine was ineffective in vivo, despite good in vitro activity (202).

More recent studies have focused on therapy with posaconazole in refractory mycoses due to $R$. mackenziei, $C$. bantiana, and $E$. dermatiditis. Al-Abdely et al. found posaconazole to be more effective than amphotericin B or itraconazole in murine models of central nervous system infection with $R$. mackenziei and $C$. bantiana $(14,15)$. Posaconazole was also found to be effective in a model of disseminated $E$. dermatiditis infection (301). In another murine model of $C$. bantiana infection, posaconazole and flucytosine improved survival alone, though the combination of posaconazole, flucytosine, and micafungin yielded the greatest benefit (481). In a recent murine model of $F$. monophora, posaconazole was associated with significantly better survival than amphotericin B or itraconazole (113). 
TABLE 4. Clinical syndromes, associated dematiaceous fungi, and suggested therapy ${ }^{a}$

\begin{tabular}{lll}
\hline \multicolumn{1}{c}{ Clinical syndrome } & \multicolumn{1}{c}{ Commonly associated fungal genera or species } & Therapy $^{b}$ \\
\hline $\begin{array}{l}\text { Eumycetoma } \\
\text { Chromoblastomycosis }\end{array}$ & $\begin{array}{l}\text { Madurella, Pyrenochaetae, Leptosphaeria } \\
\text { Fonsecaea } \text { (F. pedrosoi), Phialophora, Rhinocladiella }\end{array}$ & $\begin{array}{l}\text { Azole } \pm \text { Terb } \\
\text { Azole } \pm \text { Terb }\end{array}$ \\
$\begin{array}{l}\text { Phaeohyphomycosis } \\
\text { Allergic fungal sinusitis }\end{array}$ & Bipolaris, Curvularia & Surgery + steroids \pm Itra \\
Allergic bronchopulmonary & Bipolaris, Curvularia & Steroids \pm Itra \\
mycosis & Alternaria, Scopulariopsis & Itra or Terb \pm topical agents \\
Onychomycosis & Hortaea werneckii, Stenella araguata & Topical agents \\
Tinea nigra & Alternaria, Exophiala, Phialophora & Surgery \pm azole \\
Subcutaneous nodules & Curvularia, Bipolaris, Exserohilum & Topical natamycin \pm topical azole \\
Keratitis & Scedosporium prolificans, Alternaria & Vori \pm Terb \\
Bone and joint infection & Curvularia, Exophiala, Alternaria & Catheter removal \pm AmB or azole \\
Peritonitis & Ochroconis, Exophiala, Chaetomium & Vori (L-AmB if severe) \\
Pneumonia & Cladophialophora bantiana, Rhinocladiella & Azole + L-AmB or echinocandin \pm 5 -FC (see text) \\
Brain abscess & mackenziei, Ochroconis & Vori + Terb \pm echinocandin, Vori \pm echinocandin \\
Disseminated disease & Scedosporium prolificans, Bipolaris, Exophiala & or L-AmB (see text) \\
\hline
\end{tabular}

${ }^{a}$ Adapted from reference 625 with permission of Expert Reviews Ltd.

${ }^{b}$ Abbreviations: Vori, voriconazole; Itra, itraconazole; Terb, terbinafine; L-AmB, lipid amphotericin B; 5-FC, flucytosine; azole, voriconazole, posaconazole, or itraconazole; +, with; \pm , with or without.

Posaconazole was associated with improved survival compared with amphotericin B or caspofungin in a murine model of Exophiala infection (633).

S. prolificans was studied in a murine model, and the combination of micafungin with either voriconazole or amphotericin B was associated with improved survival, though the triple combination of all three agents was not more effective (637). Posaconazole with granulocyte-macrophage colony-stimulating factor (GM-CSF) against S. prolificans did not improve survival in one study (698), though liposomal amphotericin B with G-CSF did improve survival in a murine model (560).

\section{CLINICAL SYNDROMES AND THEIR MANAGEMENT}

A wide variety of clinical syndromes have been associated with melanized fungi, reflecting their diverse nature (Table 4). The number of published articles relating to these fungi has risen steadily in recent years. They may be considered opportunists or true pathogens, and many of the various clinical presentations can occur in both healthy and immunocompromised individuals. In reviewing the literature, it is seen that case reports often lack crucial details of medical history, diagnostic studies, therapy, and especially clinical follow-up. This limits their usefulness in determining the efficacy of the therapy. However, since randomized trials are not practical given the rarity of these infections, we are left to manage with the available data.

\section{Eumycetoma}

Mycetoma is one of the oldest infections described in recorded writings, being mentioned as "pada valmikam" (anthill foot) in the ancient Vedic hymns of India (455). Eumycetoma is due to fungi and accounts for one-third to one-half of all cases of mycetoma (473). The first report in the modern medical literature was in 1846 by Godfrey (289). It is a chronic subcutaneous infection caused by a small group of fungi and characterized by the presence of grains, or sclerotia, in tissue
(502). These grains are usually white or black, depending on the fungal species involved, and are composed of fungal cells surrounded by a dense extracellular matrix containing a melanin compound, which gives it a dark color and likely has a role in protecting the organism from host defenses (9). Eumycetoma is common in many tropical and subtropical areas of the world. The species involved are often associated with a particular geographic region. M. mycetomatis is one of the most common species, particularly in Africa and India (9). Many other species have been implicated, including Pyrenochaeta romeroi (South America), Leptosphaeria senegalensis (Africa), E. jeanselmei, Curvularia spp., and P. verrucosa (9).

In contrast to chromoblastomycosis and subcutaneous phaeohyphomycosis, which may be cured with surgical techniques alone, eumycetoma almost always requires prolonged systemic antifungal therapy in addition to surgery due to the extensive and deep tissue involvement. The most experience has been with ketoconazole and itraconazole, though itraconazole appears to have more consistent clinical activity $(9,27,30,116$, 127). Recently, reports of success using voriconazole and posaconazole have been published $(431,447,462,537)$. Therapy generally is continued for at least 3 months, though courses of 6 to 24 months or longer are often required. In refractory cases, combination therapy has also been used, adding either flucytosine or terbinafine to an active triazole $(350,447)$. Surgery can help to reduce disease burden and occasionally cure small, localized lesions that do not involve bone (30). Amphotericin B is largely ineffective and impractical given the duration of therapy that is often required (632).

\section{Chromoblastomycosis}

Chromoblastomycosis is a slowly progressive, chronic subcutaneous mycosis that is seen predominantly in tropical areas (610). Pedroso was one of the first to report of this disease in 1920 (581). The term chromoblastomycosis was introduced by Terra et al. in 1922 (739). Minor trauma typically precedes the 
lesions, though many patients do not recall this. Nodular lesions can progress over years to form large, verrucous plaques. Histopathology is characterized by the presence of muriform sclerotic bodies (Medlar bodies or "copper pennies") in tissue, which defines this condition $(232,610)$. By far the most common species is $F$. pedrosoi, followed by Phialophora verrucosa and, less commonly, Cladophialophora carrioni and Rhinocladiella aquaspersa (610). They can also cause other clinical syndromes, often leading to confusion in the literature as some authors refer to any disease caused by these fungi as "

chromoblastomycosis" (66). Other fungi have been implicated, though some reports do not clearly describe the pathognomonic features and so are questionable (594). It is not understood how and under what conditions sclerotic bodies are formed in tissue. Melanin is thought to play an important role, though other compounds such as peptidases, glycosphingolipids, and sialidase may be involved in pathogenesis as well (666).

Therapy is difficult and various modalities have been used, usually over a period of several months and even years. Besides antifungal therapy, surgery, cryotherapy, thermotherapy, and even laser therapy have been tried (90). In a large series, cryotherapy, itraconazole, or the combination resulted in the largest number of cures (88). In developing countries, where systemic antifungals are not easily available or are too expensive, the use of cryotherapy alone in a systematic manner over several months has led to good cure rates as well (126). Such physical therapies are most effective on small, localized lesions. The exact mechanism of this effect is unclear.

Antifungal therapy is essential for moderate to severe or widespread disease. As a single agent, itraconazole appears to be the most effective, and it is the agent with which there is the most clinical experience $(88,426,610,611,620)$. A variety of other treatments have also been successful, including ketoconazole, flucytosine, local heat therapy, and amphotericin B (44, $377,515)$. However, the overall cure rate was only $57 \%$ in one large series of 100 cases from Brazil, despite use of multiple modalities (515). Recently, terbinafine has been found to have in vitro and clinical activity $(91,684)$. In refractory cases, the combination of itraconazole and terbinafine has been found to be useful, and some experts recommend this as first-line therapy for moderate to severe disease $(90,317,610)$.

\section{Phaeohyphomycosis}

The remainder of clinical syndromes can be grouped under the term phaeohyphomycosis. For the purposes of this review, they will be arbitrarily divided into allergic disease, superficial infection, deep local infection, pulmonary infection, central nervous system infection, and disseminated infection.

Allergic disease. Allergic responses to dematiaceous fungi may actually represent the most common clinical manifestation of these fungi. Though asthma has many associated environmental factors, several studies have linked it with exposure to molds and to dematiaceous fungi, Alternaria spp. and Cladosporium spp. in particular $(108,278,456,555)$. The effect has also correlated with seasonal fluctuations in outdoor mold counts (555). In addition, Alternaria has been associated with severe asthma exacerbation in some individuals (541). A frequent finding is the presence of elevated Alternaria-specific IgE (456). However, most melanized fungi do not elicit such a response, and it remains unclear why only a few genera are associated with allergic disease.

(i) Allergic fungal sinusitis. Allergic fungal sinusitis is a relatively common condition, with estimates of 6 to $9 \%$ of all cases of chronic sinusitis requiring surgery (673). Patients with this condition usually present with chronic sinus symptoms that are not responsive to antibiotics. Previously, Aspergillus was thought to be the most common fungus responsible for allergic sinusitis, but it is now appreciated that disease due to dematiaceous fungi actually comprises the majority of cases $(239,674)$. Geographic variation has also been reported, with an increased incidence in the southern United States (240).

The most common species isolated are Alternaria, Bipolaris, and Curvularia, though other rare fungi (Epicoccum and Nodulisporium) have also been reported $(155,544,673)$. However, fungi are frequently isolated from normal individuals as well (668). Criteria have been suggested for this disease, and these include (i) nasal polyps; (ii) the presence of allergic mucin, containing Charcot-Leyden crystals and eosinophils; (iii) hyphal elements in the mucosa without evidence of tissue invasion, (iv) positive skin test to fungal allergens; and (v) on computed tomography (CT) scans, characteristic areas of central hyperattenuation within the sinus cavity. Not all are considered by experts to be necessary for diagnosis $(357,675)$. Diagnosis generally depends on demonstration of allergic mucin, with or without actual culture of the organism. Therapy consists of surgery to remove the mucin, which is often tenacious, and systemic steroids, though patients have been cured by surgical therapy alone $(498,631,732)$. Antifungal therapy, usually in the form of itraconazole, may play a role in reducing the requirement for steroids, but this is not routinely recommended and small, randomized studies showed no benefit when it is used as primary therapy in addition to surgery (425, 654). However, in refractory cases, itraconazole may improve outcomes $(135,680)$. Other azoles (voriconazole) have only rarely been used for this disease (223).

In rare cases, patients may present with often chronic symptoms of mass effect due to the inflammation extending from sinuses into adjacent structures, including the orbits $(102,125$, $150,379)$. These are almost always immunocompetent patients. In addition to surgery and steroids, systemic antifungal therapy is often given.

(ii) ABPM. Allergic bronchopulmonary mycosis (ABPM) is similar in presentation to allergic bronchopulmonary aspergillosis (ABPA), which is typically seen in patients with asthma or cystic fibrosis $(5,658)$. There is a suggestion that allergic fungal sinusitis and allergic bronchopulmonary mycosis may actually be a continuum of disease and should be referred to as sinobronchial allergic mycosis (SAM) (771). Criteria for the diagnosis of ABPA in patients with asthma include (i) asthma, (ii) positive skin test for fungal allergens, (iii) elevated IgE levels, (iv) Aspergillus-specific IgE, and (v) proximal bronchiectasis (5). Similar criteria for ABPM are not established but may include elevated $\mathrm{IgE}$ levels, positive skin tests, and response to systemic steroids.

In reviewing cases of $\mathrm{ABPM}$ due to dematiaceous fungi, essentially all cases are found to be due to Bipolaris or Curvularia (323, 432, 526, 622, 631, 658, 753). Asthma was common in these cases, but bronchiectasis was often not 
present, perhaps reflecting somewhat different pathogenic mechanisms. All cases had either eosinophilia or elevated IgE levels. Therapy was primarily systemic steroids, with a slow taper over 2 to 3 months or longer, if necessary. Itraconazole has been used as a steroid-sparing agent in ABPA, but its efficacy is not clear and routine use of itraconazole is not generally recommended (5).

Superficial infections. These cases of superficial infections involve only keratinized tissues, such as the fingernails and toenails and the stratum corneum. Consequences of these infections are generally cosmetic. Relatively few fungi are responsible for the majority of infections.

(i) Onychomycosis. Dematiaceous fungi are rare causes of onychomycosis. Clinical features may include a history of trauma, involvement of only one or two toenails, and lack of response to standard systemic therapy (316). Alternaria, Scopulariopsis, and Neoscytalidium have been reported, with the last genus being highly resistant to therapy $(68,316,643$, 752 ). In one study, Neoscytalidium infection was associated with plucking of green tea leaves (68). Itraconazole and terbinafine are the most commonly used systemic agents and may be combined with topical therapy for refractory cases $(316,752)$. No published data are available for the newer azole agents.

(ii) Tinea nigra. Tinea nigra is an uncommon infection confined to the stratum corneum. The characteristic appearance is that of a pigmented macule, usually on the palms or soles, and may be bilateral $(440,682)$. It is usually asymptomatic. The most common reported cause is Hortaea werneckii (previously Phaeoannellomyces werneckii), with some cases due to Stenella araguata $(87,588,682)$. Most cases are associated with exposure to sandy beaches in tropical regions, where $H$. werneckii is found in areas of high salinity (588). However, individual cases due to Scopulariopsis brevicaulis, Phoma eupyrena, and Chaetomium globosum with findings consistent with tinea nigra have also been reported $(57,154,287)$. Diagnosis is made by skin scraping, and biopsy is not needed. Although systemic antifungals have been given with success (318), topical therapy with azoles or keratolytics is very effective $(87,588)$.

Deep local infections. Deep local infections are a heterogenous group of infectious syndromes that are typically caused by local trauma. Virtually any of the melanized fungi discussed in this review may cause these infections. While they are rarely life-threatening, even in immunocompromised patients, considerable morbidity can result due to difficulties in treatment and complications.

(i) Subcutaneous lesions. Subcutaneous lesions are the most common case reports of infection due to melanized fungi in the literature. Alternaria spp. are by far the most common etiologic agent, with a recent review cataloguing over 156 cases up to 2007 (195, 191, 275, 373, 542, 577, 710, 751). Exophiala spp. and Phialophora spp. are the next most common fungi, followed by Cladosporium spp., Exserohilum spp., Veronaea botryosa, and many others with scattered case reports $(7,12,19$, $23,32,36,56,61,65,81,140,143,194,209,230,241,251,258$, $306,313,342,362,365,369,387,413,418,421,457,469,482$, 486, 487, 495, 539, 559, 556, 568, 571, 576, 590, 609, 613, 614, $646,659,694,699,720,728,749,764,776,807,818)$. Minor trauma is the usual inciting factor, though it is frequently unrecognized by the patient. Occasionally wood splinters or other vegetable matter is found upon skin biopsy or excision of the lesion $(499,524,559,660)$.

Many patients are immunocompetent, and they often are from a rural background, i.e., farmers with frequent, minor trauma from plant material or gardeners $(133,192,206,233$, $277,359,576,751,815)$. Organ transplantation is also a common risk factor $(28,38,112,142,235,236,262,271,286,296$, $465,484,514,556,587,645,679,704,725)$. Apparently nosocomial cases have also been reported, with skin irritations from dressings or i.v. sites as possible risk factors (237). Lesions typically occur on exposed areas of the body and often appear as isolated cystic or papular lesions. Presentation is usually indolent, with weeks to months of gradual enlarging mass, though pain is often absent. Severely immunocompromised patients are at increased risk of subsequent dissemination, though this may rarely occur in apparently immunocompetent patients as well. Occasionally, infection may extend to involve joints or bone, requiring more extensive surgery or prolonged antifungal therapy (198).

Multiple therapeutic options are available, usually depending on the immune status of the patient and the extent of lesions. Oral systemic therapy with an azole antifungal agent in conjunction with surgery is frequently employed and has been used successfully, particularly in immunocompromised patients. This is to prevent possible disseminated infection, though this is actually very rare in all but the most immunosuppressed patients. Terbinafine has also been used successfully, particularly in patients failing azole therapy (7). Surgical excision alone has been successful in a number of cases, even in organ transplant patients $(12,194,235,387,418,558,587$, $646,686,720,764)$. The Mohs surgical technique, which was developed for removing melanoma, may be a useful surgical approach, as it spares tissue and completely removes the pathological lesion in staged surgeries (86). Patients for whom prolonged antifungal therapy is problematic may also benefit from this technique, such as transplant patients, who often are on immunosuppressive medications that interact with oral triazole antifungals. For multiple lesions where resection may be difficult, antifungal therapy alone has also been successful, even in immunocompromised patients $(251,514)$. A variety of alternative therapies have been successfully employed as well, including cryotherapy, thermotherapy (local heat application), and supersaturated potassium iodide (SSKI) $(286,313,751)$. These are particularly relevant in developing countries where systemic antifungal therapy is difficult to obtain or too expensive for patients. Recurrences may occur several months to over a year after therapy is complete, so careful clinical follow-up is important $(38,142)$.

(ii) Keratitis. Fungal keratitis is an important ophthalmologic problem, particularly in tropical areas of the world (298). In one large series, $40 \%$ of all infectious keratitis was caused by fungi, almost exclusively molds (298). The most common fungi are Fusarium and Aspergillus, followed by dematiaceous fungi (up to 8 to $17 \%$ of cases) $(298,715)$. Many species can cause disease, with Bipolaris and Curvularia most common, though Lasiodiplodia theobromae may cause more severe disease (55, $75,213,298,743,744)$. Approximately half the cases are associated with trauma; prior eye surgery, diabetes, and contact lens use have also been noted as important risk factors (298, 
743). Diagnosis rests on potassium hydroxide $(\mathrm{KOH})$ smear and culture, with many dematiaceous fungi associated with pigmented plaques (270).

Many cases of keratitis due to dematiaceous fungi have come from India $(77,136,210,269,582)$. In a large experience of keratitis due to dematiaceous fungi, 88 cases were examined (269). The most common dematiaceous genus causing keratitis was Curvularia, followed by Bipolaris, Exserohilum, and Lasiodiplodia. Almost half the cases were associated with trauma. Most patients received topical agents only ( $5 \%$ natamycin with or without an azole), though more severe cases also received oral ketoconazole. Overall response was $72 \%$ in those available for follow-up. Surgery was needed in 13 patients, with an additional 6 requiring enucleation due to poor response. Itraconazole topically has also been used with success (582).

In a study from the United States of 43 cases of Curvularia keratitis, almost all were associated with trauma (800). Plants were the most common source, though several cases of metal injury were seen as well. Topical natamycin was used almost exclusively, with only a few severe cases requiring adjunctive therapy, usually with an azole. Of the oral agents, itraconazole had the best in vitro activity, though the majority of isolates were resistant to flucytosine. Surgery, including penetrating keratoplasty, was required in $19 \%$ of patients. At the end of therapy, only $78 \%$ had a visual acuity of $20 / 40$ or better. Other case series from the United States have noted a rise in contact lens use as a risk factor in recent years $(374,388)$. In the southern United States, cases are more frequently seen during warm, humid months (799).

Topical polyenes, such as amphotericin B and natamycin, are commonly used, but oral and topical itraconazole have been found to be useful as well $(298,744)$. Use of voriconazole has become more common, with topical preparations well described (211), but published cases involving dematiaceous fungi are infrequent (564). A series of Alternaria keratitis cases that were refractory to natamycin responded to topical azoles (758). A recent analysis of clinical trials involving fungal keratitis suggested that none of the available agents was highly effective (247). Many patients are left with residual visual deficits at the end of therapy. Clearly, further advances in therapy are needed for this debilitating disease.

(iii) Bone and joint infections. There are relatively few case reports of isolated osteoarticular infections due to dematiaceous fungi, perhaps reflecting the significant trauma often required for implantation into these deeper tissues, though some cases did not have noticeable trauma. S. prolificans is the most common cause, with all except two cases occurring in young children $(166,299,325,406,453,474,716,718)$. In addition to surgery, various antifungal therapies have been employed, with three cases using the combination of voriconazole and terbinafine with success $(166,299,406)$. Unusual therapies have also been tried, including irrigation with polyhexamethylbiguanide and oral therapy with miltefosine (406, 716). Whether these actually improved the likelihood of a clinical response is unknown.

Alternaria alternata has been reported to cause palatal ulcers with associated osteomyelitis in patients with chronic sinusitis (198, 265, 297). Although immune tolerance has been suggested in these cases, no convincing evidence is available. Re- currences were common despite prolonged antifungal therapy.

Other organisms associated with single case reports include P. obovatum, E. oligosperma, C. arxii, M. mycetomatis, $P$. richardsiae, $F$. pedrosoi, and $P$. parasiticum $(94,390,398,472,512$, $689,812)$. Itraconazole was the most common single agent used with success $(398,512,689)$. Antifungal therapy for all these cases is usually prolonged, i.e., $>6$ months and up to 2 years.

(iv) Peritonitis. Peritonitis occurs essentially only in patients receiving peritoneal dialysis $(6,8,69,97,106,115,117,144$, 197, 261, 302, 305, 367, 405, 416, 461, 466, 563, 598, 605, 618, $619,656,690,762,765,783,784)$. The presentation is usually subacute, with many patients being without significant symptoms. The genera isolated included Curvularia (eight cases); Exophiala (five); Alternaria (four); Bipolaris (four); Aureobasidium (three); and Lecythophora, Hormonema, and Phialemonium (one each). Eosinophils in peritoneal fluid were not uncommon and were associated with a variety of species. Catheter removal was considered critical, though one case with amphotericin B lock therapy in the catheter with systemic fluconazole resulted in cure with catheter retention (106). Outcomes were generally good, with only three deaths, two associated with persistent infection and the other with a retained catheter $(405,618,690)$.

(v) Miscellaneous infections. Various anecdotal cases of unusual infectious syndromes have been reported. A case of epididymitis due to E. jeanselmei was reported in a 54-year-old male who had received multiple needle aspirations for a symptomatic hydrocele (248). Surgical excision alone resulted in cure. In another case, a 5-year-old asymptomatic girl was noted to have "black grains" in her urine 3 weeks after treatment for a urinary tract infection. Hyphae and conidia identified as a Curvularia sp. were observed in a wet mount but did not grow in culture (635). She received no therapy, and the condition spontaneously resolved. E. dermatitidis was isolated from a case of otitis externa in a 19-year-old immunocompetent female, along with Pseudomonas (404). She responded to antibiotics and topical antimycotics. E. jeanselmei was isolated from esophageal brushings of a patient with Barrett's esophagus, and biopsy revealed hyphal elements as well (669). The patient was treated with ketoconazole, though little clinical improvement was noted after 5 months. A case of acute, invasive sinusitis due to $E$. rostratum was reported in an 18-year-old female with aplastic anemia and persistent neutropenia (442). She underwent surgery and prolonged antifungal therapy with liposomal amphotericin B and voriconazoleitraconazole, although she died of her underlying disease with persistent evidence of infection at autopsy. In contrast to the case for allergic sinusitis, such presentations are rare in immunocompromised patients.

Pulmonary infection. Pulmonary infection is usually seen in immunocompromised patients or those with underlying lung disease, and it may be due to a wide variety of species, including S. prolificans, C. bantiana, Chaetomium spp., Ochroconis gallopava, Exophiala spp., Alternaria, Cladophialophora boppii, F. pedrosoi, L. theobromae, Aureobasidium pullulans, Curvularia spp., Sarcinosporin inkin, and P. verrucosa (1, 17, 63, 92, 101, 107, 199, 219, 267, 303, 348, 351, 352, 402, 427, 429, 443, $464,477,520,528,554,583,701,734,736,805,814)$. Clinical manifestations include pneumonia, asymptomatic solitary pul- 
monary nodules, and endobronchial lesions which may cause hemoptysis. Therapy has consisted of systemic antifungal agents, usually amphotericin B or itraconazole initially, followed by itraconazole for a more prolonged period. Mortality rates are high in immunocompromised patients $(>40 \%)$. Experience with voriconazole is accumulating and appears promising $(17,199,219,348)$. Posaconazole was effective in a case of Alternaria pneumonia in a patient with leukemia that was refractory to amphotericin B and voriconazole (528). Occasional cases of solitary pulmonary nodules in immunocompetent patients may be cured with surgical resection alone $(92,303)$.

Central nervous system infection. Central nervous system infection is a rare but frequently fatal manifestation of phaeohyphomycosis, often in immunocompetent individuals. In a review of 101 cases of central nervous system infection due to dematiaceous fungi (628), the most common presentation was found to be brain abscess $(11,24,21,34,42,53,60,79,82,100$, $110,114,137,138,156,168,169,193,212,222,254,255,290$, $314,319,329,337,343,371,394,396,408,410,412,415,422$, $458,500,505,513,523,527,529-531,562,570,578,602,629$, $649,661,662,665,667,678,681,692,702,711,726,738,746$, $763,786,787,790,791,803,816)$. What is truly unique about this disease is that over half the cases were in patients with no risk factor or immunodeficiency. In addition, no specific exposures were associated with onset of infection, though many cases seem to occur in rural areas. Typical symptoms included headache, neurologic deficits, and seizures, though rarely all three. The most common species was $C$. bantiana, accounting for half the cases. Other species included Rhinocladiella mackenziei, Ochroconis gallopava, Bipolaris spicifera, Exophiala dermatitidis, and Chaetomium strumarium. Mortality was $>70 \%$. Since that review in 2004, over 50 cases have been reported, with $C$. bantiana remaining the most frequently seen isolate $(131,4,17,16,22,31,40,70,78,93,121,139,170,189,243$, $244,268,272,276,328,332,341,363,380,399,420,433,448$, 450, 467, 476, 545, 575, 636, 657, 696, 700, 721, 731, 733, 754, 759). A new species, Fonsecaea monophora, has been reported since that time and appears to have a predilection for causing CNS disease, in contrast to its related species, $F$. pedrosoi $(420$, 721,733 ). Encephalitis with diffuse brain involvement is a rare presentation, with essentially $100 \%$ mortality $(1,80)$.

The pathogenesis may be hematogenous spread from an initial, presumably subclinical pulmonary focus, though this remains speculation. Animal models of $C$. bantiana reliably replicate CNS infection with intravenous or intranasal inoculation, though these are generally immunosuppressed mice $(15,200)$. However, it remains unclear why these fungi preferentially cause CNS disease in immunocompetent individuals.

Meningitis has also been described, usually in immunocompromised patients $(3,17,59,74,260,366,423,444,470,729)$. However, cases with iatrogenic complications related to contaminated steroid preparations injected epidurally have been reported (131). These can be difficult to treat, and mortality is high $(>60 \%)$.

Many therapeutic strategies have been used in the treatment of brain abscess, though it is unclear if any result in significantly improved outcomes. The retrospective analysis of 101 reported cases mentioned above suggested that the combination of amphotericin B, flucytosine, and itraconazole may be associated with improved survival, though it was not frequently used.
Subsequent reports have documented various regimens, some using voriconazole or posaconazole with clinical success, though failures have also been reported. Voriconazole was unsuccessful in treating three out of four cases of $C$. bantiana brain abscess, though two of these patients were immunocompromised, and one received concomitant phenytoin, which may have reduced levels of voriconazole $(243,450,467,754)$. Despite these reports, voriconazole may have a role in therapy of phaeohyphomycotic brain abscess, as it has been successfully used in cases of Aspergillus and $S$. apiospermum brain abscess $(188,540)$. Posaconazole has been reported to be effective in a case of $R$. mackenziei brain abscess, which represents the first reported survival of infection due to this species (16). For cases due to $C$. bantiana, addition of flucytosine to azole therapy may be useful given its in vitro activity against this species specifically and in vivo and clinical data $(481,628)$. Based on the experience described above and animal studies, a combination of agents is likely to be more effective than monotherapy, though the optimal combination remains unclear and should be based on the individual case.

What does appear to be consistent is that complete excision of brain abscess whenever feasible is associated with better outcomes than aspiration or partial excision. In a series of 10 cases due to $C$. bantiana at one institution, all surviving patients were able to have complete resection of the brain lesion (268). In another case, repeated surgical excisions alone resulted in cure (189). However, outcomes remain poor, with an overall mortality of $>70 \%$.

Disseminated infection. Disseminated infection is the most uncommon manifestation of infection caused by melanized fungi. In a review of 72 cases (627), most patients were immunocompromised, though occasional patients without known immunodeficiency or risk factors developed disseminated disease as well $(3,24,73,76,95,105,119,171,208,238,242,246$, 288, 291, 308, 321, 345, 386, 389, 397, 400, 402, 403, 409, 423, 424, 435, 471, 485, 516, 538, 543, 550, 557, 578, 593, 595, 596, $612,621,627,640,647,672,688,712,713,740,755,769,772$, $794,804,806,822)$. In contrast to most invasive mold infections, blood cultures were positive in over half the cases. The most common isolate was $S$. prolificans, accounting for over a third of cases. Since that review, $S$. prolificans remains the most frequent cause of disseminated disease, almost exclusively in immunocompromised patients $(33,62,96,151,304,358,364$, $368,434,697,709,747,750,796)$. E. dermatitidis, in contrast, is commonly seen in immunocompetent patients, particularly from Asia (13, 349, 565). A variety of other molds were reported in disseminated disease, including E. oligosperma, Chaetomium perlucidum, O. gallopava, Lecythophora mutabilis, P. parasiticum, B. spicifera, Exserohilum sp., E. spinifera, and Curvularia lunata (25, 64, 85, 207, 256, 335, 417, 451, 536, 741, 788). Interestingly, peripheral eosinophilia has been observed in $9 \%$ of cases, and these were generally due to Bipolaris and Curvularia. These same species are often associated with allergic disease.

The mortality rate was $>70 \%$, despite aggressive antifungal therapy. There were no antifungal regimens associated with improved survival for disseminated infection. Scedosporium prolificans is generally resistant to all available antifungal agents, and infection with $S$. prolificans was associated with nearly $100 \%$ mortality in the absence of recovery from neu- 
tropenia, indicating the importance of the host response in this infection. However, recent case reports have suggested that the combination of itraconazole or voriconazole with terbinafine may be synergistic against this species and improve outcomes, though clinical experience is limited $(358,750,796)$. Some case reports utilized colony-stimulating factors and/or leukocyte infusions to augment antifungal therapy $(96,368,796)$.

Other combinations or therapies have not been shown to be consistently effective, though clinical experience is limited, and will likely be confined to anecdotal reports, given the rarity of this infection. Recent successful case reports have used itraconazole, voriconazole, and posaconazole for a variety of different species $(207,368,417,536,565)$. Amphotericin B alone is not generally effective $(25,64,368)$.

\section{CONCLUSIONS}

Melanized fungi remain uncommon causes of infection in humans but have become increasingly recognized in a wide variety of clinical syndromes. Many species across a broad range of genera are associated with disease, which leads to daunting challenges in diagnostic testing. However, relatively few are responsible for the majority of clinical cases. Alternaria is a frequent cause of subcutaneous lesions, Bipolaris and Curvularia are often associated with allergic disease, and C. bantiana and $S$. prolificans are the most common causes of brain abscess and disseminated disease, respectively. Taxonomy is constantly evolving as molecular methods shed new light on relationships between species. Melanin appears to be an important virulence factor for these fungi, though much additional work is needed to better understand the pathogenic mechanisms underlying these infections, particularly in immunocompetent patients. Life-threatening infections are rare but may be seen even in individuals with no apparent risk factors, especially in cases of brain abscess. As these are typically soil organisms and common laboratory contaminants, sometimes they are disregarded as nonpathogenic. However, the clinical setting in which they are isolated should always be considered when evaluating their potential as etiologic agents and before making decisions regarding therapy. Diagnosis depends on a high degree of clinical suspicion and careful mycological and pathological examination of clinical specimens. Molecular diagnostic techniques are progressing but are not standardized or reliable for the diverse species encountered.

Therapy for many infectious syndromes has evolved with the advent of several new antifungal agents in recent years. The oral triazoles voriconazole, posaconazole, and itraconazole demonstrate the most consistent in vitro activity against this group of fungi and are widely used, though voriconazole is usually the drug of choice in most clinical settings. High doses of amphotericin B lipid formulations may have a role in the treatment of refractory cases or for severe infections in unstable patients, though it is usually not effective as a single agent. Once the patient is stable, "consolidation" therapy with a broad-spectrum oral azole is often employed until a complete response is achieved. Terbinafine has broad activity against melanized fungi, and interest in its use beyond dermatophyte infections is increasing. It appears to provide synergistic activity with azole antifungals, and this may be a useful strategy against refractory subcutaneous infections such as chromoblas- tomycosis and mycetoma that often do not respond to conventional monotherapy. In addition, the use of terbinafine with voriconazole for disseminated $S$. prolificans infection has been successful with what is otherwise an almost universally fatal infection. It should be pointed out that in these disseminated cases, recovery of immune function, especially phagocytic cells, is critically important as well. Flucytosine has limited activity against dematiaceous fungi, though it may have a role in therapy of chromoblastomycosis and of brain abscess due to $C$. bantiana, in particular. Echinocandins do not appear to be useful as single agents but may be considered in combination therapy of difficult cases. Combination therapy is a potentially useful therapeutic strategy for refractory infections, particularly brain abscess and disseminated disease. However, it is not clear which antifungal drug combinations are most effective. Therapy is evolving for many of the clinical syndromes described, and randomized clinical trials to address this issue are impractical given the sporadic nature of cases. Detailed case reporting of both successful and unsuccessful clinical experiences will be important in attempting to define optimal therapy for infections caused by dematiaceous fungi.

\section{REFERENCES}

1. Abbott, S. P., L. Sigler, R. McAleer, D. A. McGough, M. G. Rinaldi, and G. Mizell. 1995. Fatal cerebral mycoses caused by the ascomycete Chaetomium strumarium. J. Clin. Microbiol. 33:2692-2698.

2. Abliz, P., K. Fukushima, K. Takizawa, and K. Nishimura. 2004. Identification of pathogenic dematiaceous fungi and related taxa based on large subunit ribosomal DNA D1/D2 domain sequence analysis. FEMS Immunol. Med. Microbiol. 40:41-49.

3. Adam, R. D., M. L. Paquin, E. A. Petersen, M. A. Saubolle, M. G. Rinaldi, J. G. Corcoran, J. N. Galgiani, and R. E. Sobonya. 1986. Phaeohyphomycosis caused by the fungal genera Bipolaris and Exserohilum. A report of 9 cases and review of the literature. Medicine 65:203-217.

4. Adeyemi, O. A., O. Lie, R. Bernstein, N. Gottardi-Littell, K. Muro, D. Patil, and G. A. Noskin. 2007. Woman with multiple brain abscesses. Clin. Infect. Dis. 45:1351-1352:1397-1399.

5. Agarwal, R. 2009. Allergic bronchopulmonary aspergillosis. Chest 135:805826.

6. Agarwal, S., N. L. Goodman, and H. H. Malluche. 1993. Peritonitis due to Exophiala jeanselmei in a patient undergoing continuous ambulatory peritoneal dialysis. Am. J. Kidney Dis. 21:673-675.

7. Agger, W. A., D. Andes, and J. W. Burgess. 2004. Exophiala jeanselmei infection in a heart transplant recipient successfully treated with oral terbinafine. Clin. Infect. Dis. 38:e112-115.

8. Ahmad, S., R. J. Johnson, S. Hillier, W. R. Shelton, and M. G. Rinaldi. 1985. Fungal peritonitis caused by Lecythophora mutabilis. J. Clin. Microbiol. 22:182-186.

9. Ahmed, A. O., W. van Leeuwen, A. Fahal, W. van de Sande, H. Verbrugh, and A. van Belkum. 2004. Mycetoma caused by Madurella mycetomatis: a neglected infectious burden. Lancet Infect. Dis. 4:566-574.

10. Ahmed, A. O., W. van Vianen, M. T. ten Kate, W. W. van de Sande, A. van Belkum, A. H. Fahal, H. A. Verbrugh, and I. A. Bakker-Woudenberg. 2003. A murine model of Madurella mycetomatis eumycetoma. FEMS Immunol. Med. Microbiol. 37:29-36.

11. Ajanee, N., M. Alam, K. Holmberg, and J. Khan. 1996. Brain abscess caused by Wangiella dermatitidis: case report. Clin. Infect. Dis. 23:197-198.

12. Ajello, L., L. K. Georg, R. T. Steigbigel, and C. J. Wang. 1974. A case of phaeohyphomycosis caused by a new species of Phialophora. Mycologia 66:490-498.

13. Alabaz, D., F. Kibar, S. Arikan, B. Sancak, U. Celik, N. Aksaray, and M. Turgut. 2009. Systemic phaeohyphomycosis due to Exophiala (Wangiella) in an immunocompetent child. Med. Mycol. 47:653-657.

14. Al-Abdely, H. M., L. Najvar, R. Bocanegra, A. Fothergill, D. Loebenberg, M. G. Rinaldi, and J. R. Graybill. 2000. SCH 56592, amphotericin B, or itraconazole therapy of experimental murine cerebral phaeohyphomycosis due to Ramichloridium obovoideum ("Ramichloridium mackenziei"). Antimicrob. Agents Chemother. 44:1159-1162.

15. Al-Abdely, H. M., L. K. Najvar, R. Bocanegra, and J. R. Graybill. 2005. Antifungal therapy of experimental cerebral phaeohyphomycosis due to Cladophialophora bantiana. Antimicrob. Agents Chemother. 49:1701-1707.

16. Al Abdely, H. M., A. M. Alkhunaizi, J. A. Al Tawfiq, M. Hassounah, M. G. Rinaldi, and D. A. Sutton. 2005. Successful therapy of cerebral phaeohy- 
phomycosis due to Ramichloridium mackenziei with the new triazole posaconazole. Med. Mycol. 43:91-95.

17. Al-Aidaroos, A., I. Bin-Hussain, H. El Solh, A. Kofide, S. Thawadi, A Belgaumi, and A. Al Ahmari. 2007. Invasive Chaetomium infection in two immunocompromised pediatric patients. Pediatr. Infect. Dis. J. 26:456-458.

18. Al-Astruey-Izquierdo, A., M. Cuenca-Estrella, A. Monzon, and J. L. Rodriguez-Tudela. 2007. Prevalence and susceptibility testing of new species of Pseudallescheria and Scedosporium in a collection of clinical mold isolates. Antimicrob. Agents Chemother. 51:748-751.

19. Al-Attar, A., C. G. Williams, and R. J. Redett. 2006. Rare lower extremity invasive fungal infection in an immunosuppressed patient: Exserohilum longirostratum. Plast. Reconstr. Surg. 117:e44-47.

20. Alcorn, J. L. 1983. Generic concepts in Drechslera, Bipolaris and Exserohilum. Mycotaxon 17:1-86.

21. Aldape, K. D., H. S. Fox, J. P. Roberts, N. L. Ascher, J. R. Lake, and H. A Rowley. 1991. Cladosporium trichoides cerebral phaeohyphomycosis in liver transplant recipient. Report of a case. Am. J. Clin. Pathol. 95:499-502.

22. Alhabib, K. F., and E. A. Bryce. 2003. Xylohypha bantiana multiple brain abscesses in a patient with systemic lupus erythematosus. Can. J. Infect. Dis. 14:119-120.

23. Allred, B. J. 1990. Subcutaneous phaeohyphomycosis due to Exophiala jeanselmei in an immunosuppressed patient: case report. New Zealand Med. J. 103:321-322.

24. Al-Mohsen, I. Z., D. A. Sutton, L. Sigler, E. Almodovar, N. Mahgoub, H. Frayha, S. Al-Hajjar, M. G. Rinaldi, and T. J. Walsh. 2000. Acrophialophora fusispora brain abscess in a child with acute lymphoblastic leukemia: review of cases and taxonomy. J. Clin. Microbiol. 38:4569-4576.

25. Al-Obaid, I., S. Ahmad, Z. U. Khan, B. Dinesh, and H. M. Hejab. 2006. Catheter-associated fungemia due to Exophiala oligosperma in a leukemic child and review of fungemia cases caused by Exophiala species. Eur. J. Clin. Microbiol. Infect. Dis. 25:729-732.

26. Al-Rajhi, A. A., A. H. Awad, S. S. al-Hedaithy, R. K. Forster, and K. C Caldwell. 1993. Scytalidium dimidiatum fungal endophthalmitis. Br. J. Ophthalmol. 77:388-390.

27. Al-Tawfiq, J. A., and S. S. Amr. 2009. Madura leg due to Exophiala jeanselmei successfully treated with surgery and itraconazole therapy. Med. Mycol. 47:648-652.

28. Altomare, G. F., G. L. Capella, V. Boneschi, and M. A. Viviani. 2000 Effectiveness of terbinafine in cutaneous alternariosis. Br. J. Dermatol. 142:840-841.

29. Alvarez, M., P. B. Lopez, C. Rayon, G. J. Garcia, M. C. Roson Porto, M. Gonzalez, J. V. Martinez-Suarez, and J. L. Rodriguez-Tudela. 1995. Nosocomial outbreak caused by Scedosporium prolificans (inflatum): four fatal cases in leukemic patients. J. Clin. Microbiol. 33:3290-3295.

30. Ameen, M., and R. Arenas. 2009. Developments in the management of mycetomas. Clin. Exp. Dermatol. 34:1-7.

31. Amr, S. S., and J. A. Al-Tawfiq. 2007. Aspiration cytology of brain abscess from a fatal case of cerebral phaeohyphomycosis due to Ramichloridium mackenziei. Diagn. Cytopathol. 35:695-699.

32. Anandan, V., V. Nayak, S. Sundaram, and P. Srikanth. 2008. An association of Alternaria alternata and Scopulariopsis brevicaulis in cutaneous phaeohyphomycosis. Indian J. Dermatol. Venereol. Leprol. 74:244-247.

33. Ananda-Rajah, M. R., A. Grigg, and M. A. Slavin. 2008. Breakthrough disseminated Scedosporium prolificans infection in a patient with relapsed leukaemia on prolonged voriconazole followed by posaconazole prophylaxis. Mycopathologia 166:83-86.

34. Anandi, V., T. J. John, A. Walter, J. C. Shastry, M. K. Lalitha, A. A. Padhye, L. Ajello, and F. W. Chandler. 1989. Cerebral phaeohyphomycosis caused by Chaetomium globosum in a renal transplant recipient. J. Clin. Microbiol. 27:2226-2229.

35. Andrade, T. S., L. G. Castro, R. S. Nunes, V. M. Gimenes, and A. E. Cury 2004. Susceptibility of sequential Fonsecaea pedrosoi isolates from chromoblastomycosis patients to antifungal agents. Mycoses 47:216-221.

36. Aoyama, Y., M. Nomura, S. Yamanaka, Y. Ogawa, and Y. Kitajima. 2009. Subcutaneous phaeohyphomycosis caused by Exophiala xenobiotica in a non-Hodgkin lymphoma patient. Med. Mycol. 47:95-99.

37. Aquino, V. M., J. M. Norvell, K. Krisher, and M. M. Mustafa. 1995. Fatal disseminated infection due to Exserohilum rostratum in a patient with aplastic anemia: case report and review. Clin. Infect. Dis. 20:176-178.

38. Ara, M., C. Aspiroz, P. Zaballos, V. Alcalde, R. Alvarez, A. Rezusta, and J. A. Gimenez. 2006. Relapse of cutaneous Alternaria infectoria in a renal transplant recipient after 2 years. Acta Derm. Venereol. 86:154-155.

39. Arango, M., C. Jaramillo, A. Cortes, and A. Restrepo. 1998. Auricular chromoblastomycosis caused by Rhinocladiella aquaspersa. Med. Mycol. 36: $43-45$

40. Aribandi, M., C. Bazan III, and M. G. Rinaldi. 2005. Magnetic resonance imaging findings in fatal primary cerebral infection due to Chaetomium strumarium. Australas. Radiol. 49:166-169.

41. Arthur, S., L. L. Steed, D. J. Apple, Q. Peng, G. Howard, and M. EscobarGomez. 2001. Scedosporium prolificans keratouveitis in association with contact lens retained intraocularly over a long term. J. Clin. Microbiol. 39:4579-4582.
42. Arunkumar, M. J., V. Rajshekhar, M. J. Chandy, P. P. Thomas, and C. K. Jacob. 2000. Management and outcome of brain abscess in renal transplant recipients. Postgrad. Med. J. 76:207-211.

43. Arzanlou, M., J. Z. Groenewald, W. Gams, U. Braun, H. D. Shin, and P. W. Crous. 2007. Phylogenetic and morphotaxonomic revision of Ramichloridium and allied genera. Stud. Mycol. 58:57-93.

44. Attapattu, M. C. 1997. Chromoblastomycosis-a clinical and mycological study of 71 cases from Sri Lanka. Mycopathologia 137:145-151.

45. Attili, D. S., G. S. De Hoog, and A. A. Pizzirani-Kleiner. 1998. rDNA-RFLP and ITS1 sequencing of species of the genus Fonsecaea, agents of chromoblastomycosis. Med. Mycol. 36:219-225.

46. Aviv, J. E., W. Lawson, E. J. Bottone, V. P. Sachdev, P. M. Som, and H. F. Biller. 1990. Multiple intracranial mucoceles associated with phaeohyphomycosis of the paranasal sinuses. Arch. Otolaryngol. Head Neck Surg. 116:1210-1213

47. Badali, H., V. O. Carvalho, V. Vicente, D. Attili-Angelis, I. B. Kwiatkowski, A. H. Gerrits Van Den Ende, and G. S. De Hoog. 2009. Cladophialophora saturnica sp. nov., a new opportunistic species of Chaetothyriales revealed using molecular data. Med. Mycol. 47:51-62.

48. Badali, H., J. Chander, S. Bansal, A. Aher, S. S. Borkar, J. F. Meis, and G. S. De Hoog. 2010. First autochthonous case of Rhinocladiella mackenziei cerebral abscess outside the Middle East. J. Clin. Microbiol. 48:646-649.

49. Badali, H., G. S. De Hoog, I. Curfs-Breuker, B. Andersen, and J. F. Meis. 2009. In vitro activities of eight antifungal drugs against 70 clinical and environmental isolates of Alternaria species. J. Antimicrob. Chemother. 63:1295-1297.

50. Badali, H., G. S. de Hoog, I. Curfs-Breuker, and J. F. Meis. 2010. In vitro activities of antifungal drugs against Rhinocladiella mackenziei, an agent of fatal brain infection. J. Antimicrob. Chemother. 65:175-177.

51. Badali, H., C. Gueidan, M. J. Najafzadeh, A. Bonifaz, A. H. van den Ende, and G. S. de Hoog. 2008. Biodiversity of the genus Cladophialophora. Stud. Mycol. 61:175-191.

52. Badali, H., M. J. Najafzadeh, M. Van Esbroeck, E. van den Enden, B. Tarazooie, J. F. Meis, and G. S. de Hoog. 2010. The clinical spectrum of Exophiala jeanselmei, with a case report and in vitro antifungal susceptibility of the species. Med. Mycol. 48:318-327.

53. Baddley, J. W., S. A. Moser, D. A. Sutton, and P. G. Pappas. 2000. Microascus cinereus (anamorph Scopulariopsis) brain abscess in a bone marrow transplant recipient. J. Clin. Microbiol. 38:395-397.

54. Baddley, J. W., L. Mostert, R. C. Summerbell, and S. A. Moser. 2006 Phaeoacremonium parasiticum infections confirmed by beta-tubulin sequence analysis of case isolates. J. Clin. Microbiol. 44:2207-2211.

55. Badenoch, P. R., C. L. Halliday, D. H. Ellis, K. J. Billing, and R. A. Mills. 2006. Ulocladium atrum keratitis. J. Clin. Microbiol. 44:1190-1193.

56. Baker, J. G., I. F. Salkin, P. Forgacs, J. H. Haines, and M. E. Kemna. 1987. First report of subcutaneous phaeohyphomycosis of the foot caused by Phoma minutella. J. Clin., Microbiol. 25:2395-2397.

57. Bakerspigel, A. 1970. The isolation of Phoma hibernica from a lesion on a leg. Sabouraudia 7:261-264.

58. Balajee, S. A., A. M. Borman, M. E. Brandt, J. Cano, M. Cuenca-Estrella, E. Dannaoui, J. Guarro, G. Haase, C. C. Kibbler, W. Meyer, K. O'Donnell, C. A. Petti, J. L. Rodriguez-Tudela, D. Sutton, A. Velegraki, and B. L. Wickes. 2009. Sequence-based identification of Aspergillus, Fusarium, and Mucorales species in the clinical mycology laboratory: where are we and where should we go from here? J. Clin. Microbiol. 47:877-884.

59. Banerjee, T. K., A. K. Patwari, R. Dutta, V. K. Anand, and A. Chabra. 2002. Cladosporium bantianum meningitis in a neonate. Indian J. Pediatr. 69:721723.

60. Banerjee, U., A. K. Mohapatra, C. Sarkar, and R. Chaudhery. 1989. Cladosporiosis (cerebral phaeohyphomycosis) of brain-a case report. Mycopathologia 105:163-166.

61. Baradkar, V. P., M. Mathur, and S. Kumar. 2009. Phaeohyphomycosis of subcutaneous tissue caused by Phaeoacremonium parasiticum. Indian J. Med. Microbiol. 27:66-69.

62. Barbaric, D., and P. J. Shaw. 2001. Scedosporium infection in immunocompromised patients: successful use of liposomal amphotericin B and itraconazole. Med. Pediatr. Oncol. 37:122-125.

63. Barenfanger, J., F. Ramirez, R. P. Tewari, and L. Eagleton. 1989. Pulmonary phaeohyphomycosis in a patient with hemoptysis. Chest 95:1158-1160.

64. Barron, M. A., D. A. Sutton, R. Veve, J. Guarro, M. Rinaldi, E. Thompson, P. J. Cagnoni, K. Moultney, and N. E. Madinger. 2003. Invasive mycotic infections caused by Chaetomium perlucidum, a new agent of cerebral phaeohyphomycosis. J. Clin. Microbiol. 41:5302-5307.

65. Bartolome, B., R. Valks, J. Fraga, V. Buendia, J. Fernandez-Herrera, and A. Garcia-Diez. 1999. Cutaneous alternariosis due to Alternaria chlamydospora after bone marrow transplantation. Acta Dermatol. Venereol. 79:244.

66. Barton, K., D. Miller, and S. C. Pflugfelder. 1997. Corneal chromoblastomycosis. Cornea 16:235-239.

67. Bartynski, J. M., T. V. McCaffrey, and E. Frigas. 1990. Allergic fungal sinusitis secondary to dermatiaceous fungi-Curvularia lunata and Alterna ria. Otolaryngol. Head Neck Surg. 103:32-39.

68. Barua, P., S. Barua, B. Borkakoty, and J. Mahanta. 2007. Onychomycosis 
by Scytalidium dimidiatum in green tea leaf pluckers: report of two cases. Mycopathologia 164:193-195.

69. Bava, A. J., A. Fayad, C. Cespedes, and M. Sandoval. 2003. Fungal peritonitis caused by Bipolaris spicifera. Med. Mycol. 41:529-531.

70. Beeram, V., S. Challa, and P. Vannemreddy. 2008. Cerebral mycetoma with cranial osteomyelitis. J. Neurosurg. Pediatr. 1:493-495.

71. Ben-Ami, R., P. R. Lasala, R. E. Lewis, and D. P. Kontoyiannis. 2010. Lack of galactomannan reactivity in dematiaceous molds recovered from cancer patients with phaeohyphomycosis. Diagn. Microbiol. Infect. Dis. 66:200203.

72. Ben-Ami, R., R. E. Lewis, I. I. Raad, and D. P. Kontoyiannis. 2009. Phaeohyphomycosis in a tertiary care cancer center. Clin. Infect. Dis. 48:10331041.

73. Benne, C. A., C. Neeleman, M. Bruin, G. S. De Hoog, and A. Fleer. 1993. Disseminating infection with Scytalidium dimidiatum in a granulocytopenic child. Eur. J. Clin. Microbiol. Infect. Dis. 12:118-121.

74. Bennett, J. E., H. Bonner, A. E. Jennings, and R. I. Lopez. 1973. Chronic meningitis caused by Cladosporium trichoides. Am. J. Clin. Pathol. 59:398 407.

75. Ben-Simon, G. J., I. S. Barequet, and A. Grinbaum. 2002. More than tears in your eyes (Exophiala jeanselmei keratitis). Cornea 21:230-231.

76. Berenguer, J., J. L. Rodriguez-Tudela, C. Richard, M. Alvarez, M. A. Sanz, L. Gaztelurrutia, J. Ayats, and J. V. Martinez-Suarez. 1997. Deep infections caused by Scedosporium prolificans. A report on 16 cases in Spain and a review of the literature. Scedosporium prolificans Spanish Study Group. Medicine 76:256-265.

77. Bharathi, M. J., R. Ramakrishnan, S. Vasu, R. Meenakshi, and R. Palaniappan. 2003. Epidemiological characteristics and laboratory diagnosis of fungal keratitis. A three-year study. Indian J. Ophthalmol. 51:315321.

78. Bhat, S. V., D. L. Paterson, M. G. Rinaldi, and P. J. Veldkamp. 2007. Scedosporium prolificans brain abscess in a patient with chronic granulomatous disease: successful combination therapy with voriconazole and terbinafine. Scand. J. Infect. Dis. 39:87-90.

79. Bhatia, R., P. Tandon, and N. K. Misra. 1986. Inflammatory lesions of the basal ganglia and thalamus: review of twenty-one cases. Neurosurgery 19: 983-988.

80. Biggs, P. J., R. L. Allen, J. M. Powers, and H. P. Holley, Jr. 1986. Phaeohyphomycosis complicating compound skull fracture. Surg. Neurol. 25:393396.

81. Bilu, D., S. Movahedi-Lankarani, R. A. Kazin, C. Shields, and M. Moresi. 2004. Cutaneous Bipolaris infection in a neutropenic patient with acute lymphoblastic leukemia. J. Cutan. Med. Surg. 8:446-449.

82. Binford, C. H., R. K. Thompson, M. E. Gorham, and C. W. Emmons. 1952. Mycotic brain abscess due to Cladosporium trichoides, a new species. Am. J. Clin. Pathol. 22:535-542.

83. Bloomfield, B. J., and M. Alexander. 1967. Melanins and resistance of fungi to lysis. J. Bacteriol. 93:1276-1280.

84. Boerema, G. H., J. de Gruyter, M. E. Noordeloos, and M. E. C. Hamers. 2004. Phoma identification manual. Differentiation of specific and infraspecific taxa in culture. CABI Publishing, Cambridge, MA.

85. Boggild, A. K., S. M. Poutanen, S. Mohan, and M. A. Ostrowski. 2006. Disseminated phaeohyphomycosis due to Ochroconis gallopavum in the setting of advanced HIV infection. Med. Mycol. 44:777-782.

86. Bogle, M. A., M. S. Rabkin, and A. K. Joseph. 2004. Mohs micrographic surgery for the eradication of phaeohyphomycosis of the hand. Dermatol. Surg. 30:231-233.

87. Bonifaz, A., H. Badali, G. S. de Hoog, M. Cruz, J. Araiza, M. A. Cruz, L. Fierro, and R. M. Ponce. 2008. Tinea nigra by Hortaea werneckii, a report of 22 cases from Mexico. Stud. Mycol. 61:77-82.

88. Bonifaz, A., E. Carrasco-Gerard, and A. Saul. 2001. Chromoblastomycosis: clinical and mycologic experience of 51 cases. Mycoses 44:1-7.

89. Bonifaz, A., S. De Hoog, M. R. McGinnis, A. Saul, O. Rodriguez-Cortes, J. Araiza, M. Cruz, and P. Mercadillo. 2009. Eumycetoma caused by Cladophialophora bantiana successfully treated with itraconazole. Med. Mycol. 47:111-114

90. Bonifaz, A., V. Paredes-Solis, and A. Saul. 2004. Treating chromoblastomycosis with systemic antifungals. Expert. Opin. Pharmacother. 5:247-254.

91. Bonifaz, A., A. Saul, V. Paredes-Solis, J. Araiza, and L. Fierro-Arias. 2005. Treatment of chromoblastomycosis with terbinafine: experience with four cases. J. Dermatolog. Treat. 16:47-51.

92. Borges, M. C., Jr., S. Warren, W. White, and E. V. Pellettiere. 1991. Pulmonary phaeohyphomycosis due to Xylohypha bantiana. Arch. Pathol. Lab. Med. 115:627-629.

93. Borkar, S. A., M. S. Sharma, G. Rajpal, M. Jain, I. Xess, and B. S. Sharma. 2008. Brain abscess caused by Cladophialophora bantiana in an immunocompetent host: need for a novel cost-effective antifungal agent. Indian J. Med. Microbiol. 26:271-274.

94. Bossler, A. D., S. S. Richter, A. J. Chavez, S. A. Vogelgesang, D. A. Sutton, A. M. Grooters, M. G. Rinaldi, G. S. de Hoog, and M. A. Pfaller. 2003. Exophiala oligosperma causing olecranon bursitis. J. Clin. Microbiol. 41: 4779-4782.
95. Bourbeau, P., D. A. McGough, H. Fraser, N. Shah, and M. G. Rinaldi. 1992 Fatal disseminated infection caused by Myceliophthora thermophila, a new agent of mycosis: case history and laboratory characteristics. J. Clin. Microbiol. 30:3019-3023.

96. Bouza, E., P. Munoz, L. Vega, M. Rodriguez-Creixems, J. Berenguer, and A. Escudero. 1996. Clinical resolution of Scedosporium prolificans fungemia associated with reversal of neutropenia following administration of granulocyte colony-stimulating factor. Clin. Infect. Dis. 23:192-193.

97. Brackett, R. W., A. N. Shenouda, S. S. Hawkins, and W. B. Brock. 1988 Curvularia infection complicating peritoneal dialysis. South. Med. J. 81: 943-944.

98. Brandt, M. E., and D. W. Warnock. 2003. Epidemiology, clinical manifestations, and therapy of infections caused by dematiaceous fungi. J. Chemother. 15(Suppl. 2):36-47.

99. Brasch, J., J. O. Busch, and G. S. de Hoog. 2008. Cutaneous phaeohyphomycosis caused by Alternaria infectoria. Acta Dermatol. Venereol. 88:160161.

100. Brown, J. W., III, J. Nadell, C. V. Sanders, and L. Sardenga. 1976. Brain abscess caused by Cladosporium trichoides (bantianum): a case with paranasal sinus involvement. South. Med. J. 69:1519-1521.

101. Brubaker, L. H., J. C. Steele, Jr., and J. P. Rissing. 1988. Cure of Curvularia pneumonia by amphotericin B in a patient with megakaryocytic leukemia. Arch. Pathol. Lab. Med. 112:1178-1179.

102. Brummund, W., V. P. Kurup, G. J. Harris, J. A. Duncavage, and J. A. Arkins. 1986. Allergic sino-orbital mycosis. A clinical and immunologic study. JAMA 256:3249-3253.

103. Brush, L., and N. P. Money. 1999. Invasive hyphal growth in Wangiella dermatitidis is induced by stab inoculation and shows dependence upon melanin biosynthesis. Fungal Genet. Biol. 28:190-200.

104. Bryan, C. S., C. W. Smith, D. E. Berg, and R. B. Karp. 1993. Curvularia lunata endocarditis treated with terbinafine: case report. Clin. Infect. Dis. 16:30-32.

105. Bryan, M. G., D. M. Elston, C. Hivnor, and B. A. Honl. 2000. Phaeohyphomycosis in a premature infant. Cutis 65:137-140.

106. Buchanan, W. E., M. J. Quinn, and J. A. Hasbargen. 1994. Peritoneal catheter colonization with Alternaria: successful treatment with catheter preservation. Perit. Dial. Int. 14:91-92.

107. Burns, K. E., N. P. Ohori, and A. T. Iacono. 2000. Dactylaria gallopava infection presenting as a pulmonary nodule in a single-lung transplant recipient. J. Heart Lung Transplant. 19:900-902.

108. Bush, R. K., and J. J. Prochnau. 2004. Alternaria-induced asthma. J. Allergy Clin. Immunol. 113:227-234.

109. Butler, M. J., and A. W. Day. 1998. Fungal melanins: a review. Can. J. Microbiol. 44:1115-1136.

110. Buxi, T. B., K. Prakash, R. Vohra, and D. Bhatia. 1996. Imaging in phaeohyphomycosis of the brain: case report. Neuroradiology 38:139-141.

111. Caligiorne, R. B., M. A. Resende, P. H. Melillo, C. P. Peluso, F. H. Carmo, and V. Azevedo. 1999. In vitro susceptibility of chromoblastomycosis and phaeohyphomycosis agents to antifungal drugs. Med. Mycol. 37:405-409.

112. Calista, D., M. Leardini, and F. Arcangeli. 2003. Subcutaneous Exophiala jeanselmei infection in a heart transplant patient. Eur. J. Dermatol. 13:489.

113. Calvo, E., F. J. Pastor, M. M. Rodriguez, E. Mayayo, V. Salas, and J. Guarro. 2010. Murine model of a disseminated infection by the novel fungus Fonsecaea monophora and successful treatment with posaconazole. Antimicrob. Agents Chemother. 54:919-923.

114. Campbell, C. K., and S. S. A. Al-Hedaithy. 1993. Phaeohyphomycosis of the brain caused by Ramichloridium mackenziei sp. nov. in Middle Eastern countries. J. Med. Vet. Mycol. 31:325-332.

115. Canon, H. L., S. C. Buckingham, R. J. Wyatt, and D. P. Jones. 2001. Fungal peritonitis caused by Curvularia species in a child undergoing peritoneal dialysis. Pediatr. Nephrol. 16:35-37.

116. Capoor, M. R., G. Khanna, D. Nair, A. Hasan, Rajni, M. Deb, and P. Aggarwal. 2007. Eumycetoma pedis due to Exophiala jeanselmei. Indian J. Med. Microbiol. 25:155-157.

117. Caporale, N. E., L. Calegari, D. Perez, and E. Gezuele. 1996. Peritoneal catheter colonization and peritonitis with Aureobasidium pullulans. Perit. Dial. Int. 16:97-98.

118. Cappelletty, D., and K. Eiselstein-McKitrick. 2007. The echinocandins. Pharmacotherapy 27:369-388.

119. Carreter de Granda, M. E., C. Richard, E. Conde, A. Iriondo, F. Marco de Lucas, R. Salesa, and A. Zubizarreta. 2001. Endocarditis caused by Scedosporium prolificans after autologous peripheral blood stem cell transplantation. Eur. J. Clin. Microbiol. Infect. Dis. 20:215-217.

120. Carrillo, A. J., and J. Guarro. 2001. In vitro activities of four novel triazoles against Scedosporium spp. Antimicrob. Agents Chemother. 45:2151-2153.

121. Carter, E., and C. Boudreaux. 2004. Fatal cerebral phaeohyphomycosis due to Curvularia lunata in an immunocompetent patient. J. Clin. Microbiol. 42:5419-5423.

122. Carzaniga, R., D. Fiocco, P. Bowyer, and R. J. O'Connell. 2002. Localization of melanin in conidia of Alternaria alternata using phage display antibodies. Mol. Plant Microbe Interact. 15:216-224. 
123. Casadevall, A., A. L. Rosas, and J. D. Nosanchuk. 2000. Melanin an virulence in Cryptococcus neoformans. Curr. Opin. Microbiol. 3:354-358.

124. Castanet, J., J. P. Lacour, M. Toussaint-Gary, C. Perrin, S. Rodot, and J. P. Ortonne. 1995. Alternaria tenuissima plurifocal cutaneous infection. Ann. Dermatol. Venereol. 122:115-118.

125. Castelnuovo, P., F. De Bernardi, C. Cavanna, F. Pagella, P. Bossolesi, P. Marone, and C. Farina. 2004. Invasive fungal sinusitis due to Bipolaris hawaiiensis. Mycoses 47:76-81.

126. Castro, L. G., E. R. Pimentel, and C. S. Lacaz. 2003. Treatment of chromomycosis by cryosurgery with liquid nitrogen: 15 years' experience. Int. J. Dermatol. 42:408-412.

127. Castro, L. G., and J. Piquero-Casals. 2008. Clinical and mycologic finding and therapeutic outcome of 27 mycetoma patients from Sao Paulo, Brazil. Int. J. Dermatol. 47:160-163.

128. Cateau, E., T. Mergey, C. Kauffmann-Lacroix, and M. H. Rodier. 2009 Relationships between free living amoebae and Exophiala dermatitidis: a preliminary study. Med. Mycol. 47:115-118.

129. Celard, M., E. Dannaoui, M. A. Piens, E. Gueho, G. Kirkorian, T. Greenland, F. Vandenesch, and S. Picot. 1999. Early Microascus cinereus endocarditis of a prosthetic valve implanted after Staphylococcus aureus endocarditis of the native valve. Clin. Infect. Dis. 29:691-692.

130. Centers for Disease Control and Prevention. 2009. Biosafety in microbiological and biomedical laboratories, 5th ed., p. 170-177. Centers for Disease Control and Prevention, Atlanta, GA.

131. Centers for Disease Control and Prevention. 2002. Exophiala infection from contaminated injectable steroids prepared by a compounding pharmacy-United States, July-November 2002. MMWR Morb. Mortal. Wkly. Rep. 51:1109-1112.

132. Cermeno-Vivas, J. R., J. M. Torres-Rodriguez, J. C. Fung-Tomc, E. Huczko, B. Minassian, and D. P. Bonner. 2001. In vitro susceptibility of dematiaceous fungi to ten antifungal drugs using an agar diffusion test. Rev. Iberoam. Micol. 18:113-117.

133. Chabasse, D., C. de Bievre, E. Legrand, J. P. Saint-Andre, L. de Gentile, B. Cimon, and J. P. Bouchara. 1995. Subcutaneous abscess caused by Pleurophomopsis lignicola Petr: first case. J. Med. Vet. Mycol. 33:415-417.

134. Chai, L. Y., M. G. Netea, J. Sugui, A. G. Vonk, W. W. van de Sande, A Warris, K. J. Kwon-Chung, and B. J. Kullberg. 2009. Aspergillus fumigatus conidial melanin modulates host cytokine response. Immunobiology [Epub ahead of print.] doi:10.1016/j.imbio.2009.10.002.

135. Chan, K. O., K. A. Genoway, and A. R. Javer. 2008. Effectiveness of itraconazole in the management of refractory allergic fungal rhinosinusitis. J. Otolaryngol. Head Neck Surg. 37:870-874.

136. Chander, J., N. Singla, N. Agnihotri, S. K. Arya, and A. Deep. 2008 Keratomycosis in and around Chandigarh: a five-year study from a north Indian tertiary care hospital. Indian J. Pathol. Microbiol. 51:304-306.

137. Chandramukhi, A., M. G. Ramadevi, and S. K. Shankar. 1983. Cerebra cladosporiosis—a neuropathological and microbiological study. Clin. Neurol. Neurosurg. 85:245-253.

138. Chang, C. L., D. S. Kim, D. J. Park, H. J. Kim, C. H. Lee, and J. H. Shin 2000. Acute cerebral phaeohyphomycosis due to Wangiella dermatitidis accompanied by cerebrospinal fluid eosinophilia. J. Clin. Microbiol. 38:19651966.

139. Chang, X., R. Li, J. Yu, X. Bao, and J. Qin. 2009. Phaeohyphomycosis of the central nervous system caused by Exophiala dermatitidis in a 3-year-old immunocompetent host. J. Child Neurol. 24:342-345.

140. Chen, Y. T., H. C. Lin, C. C. Huang, and Y. H. Lo. 2006. Cutaneous phaeohyphomycosis caused by an itraconazole and amphotericin B resistant strain of Veronaea botryosa. Int. J. Dermatol. 45:429-432.

141. Cherian, R. S., M. Betty, M. T. Manipadam, V. M. Cherian, P. M. Poonnoose, A. T. Oommen, and R. A. Cherian. 2009. The "dot-in-circle" sign-a characteristic MRI finding in mycetoma foot: a report of three cases. Br. J. Radiol. 82:662-665.

142. Chua, J. D., S. M. Gordon, J. Banbury, G. S. Hall, and G. W. Procop. 2001 Relapsing Exophiala jeanselmei phaeohyphomycosis in a lung-transplant patient. Transplant. Infect. Dis. 3:235-238.

143. Chuan, M. T., and M. C. Wu. 1995. Subcutaneous phaeohyphomycosis caused by Exophiala jeanselmei: successful treatment with itraconazole. Int. J. Dermatol. 34:563-566.

144. Clark, E. C., S. M. Silver, G. E. Hollick, and M. G. Rinaldi. 1995. Continuous ambulatory peritoneal dialysis complicated by Aureobasidium pullulans peritonitis. Am. J. Nephrol. 15:353-355.

145. Cleary, J. D. 2009. Echinocandins: pharmacokinetic and therapeutic issues Curr. Med. Res. Opin. 25:1741-1750.

146. Clinical Laboratory Standards Institute. 2008. Interpretive criteria for identification of bacteria and fungi by sequencing; approved guideline. CLSI MM18-A. Clinical Laboratory Standards Institute, Wayne, PA.

147. Clinical Laboratory Standards Institute. 2008. Reference method for broth dilution antifungal susceptibility testing of conidium-forming filamentous fungi. Revised M38-A2. Clinical Laboratory Standards Institute, Wayne, PA.

148. Clinical Laboratory Standards Institute. 2008. Reference method for broth dilution antifungal susceptibility testing of yeasts. Approved standard M27A3, 3rd ed. Clinical Laboratory Standards Institute, Wayne, PA.

149. Coldiron, B. M., E. L. Wiley, and M. G. Rinaldi. 1990. Cutaneous phaeohyphomycosis caused by a rare fungal pathogen, Hormonema dematioides: successful treatment with ketoconazole. J. Am. Acad. Dermatol. 23:363 367.

150. Colton, R., A. Zeharia, B. Karmazyn, N. Buller, Y. Levy, R. Inbal, S. Zmira, and E. Yaniv. 2002. Exserohilum sinusitis presenting as proptosis in a healthy adolescent male. J. Adolesc. Health 30:73-75.

151. Cooley, L., D. Spelman, K. Thursky, and M. Slavin. 2007. Infection with Scedosporium apiospermum and $S$. prolificans, Australia. Emerg. Infect. Dis. 13:1170-1177.

152. Corrado, M. L., M. Kramer, M. Cummings, and R. H. Eng. 1982. Susceptibility of dematiaceous fungi to amphotericin B, miconazole, ketoconazole, flucytosine and rifampin alone and in combination. Sabouraudia 20:109113.

153. Cortez, K. J., E. Roilides, F. Quiroz-Telles, J. Meletiadis, C. Antachopoulos, T. Knudsen, W. Buchanan, J. Milanovich, D. A. Sutton, A. Fothergill, M. G. Rinaldi, Y. R. Shea, T. Zaoutis, S. Kottilil, and T. J. Walsh. 2008 Infections caused by Scedosporium spp. Clin. Microbiol. Rev. 21:157-197.

154. Costa, A. R., E. Porto, C. d. Lacaz, N. T. de Melo, M. d. Calux, and N. Y. Valente. 1988. Cutaneous and ungual phaeohyphomycosis caused by species of Chaetomium Kunze (1817) ex Fresenius, 1829. J. Med. Vet. Mycol. 26:261-268.

155. Cox, G. M., W. A. Schell, R. L. Scher, and J. R. Perfect. 1994. First report of involvement of Nodulisporium species in human disease. J. Clin. Microbiol. 32:2301-2304.

156. Crichlow, D. K., F. T. Enrile, and M. Y. Memon. 1973. Cerebellar abscess due to Cladosporium trichoides (bantianum): case report. Am. J. Clin. Pathol. 60:416-421.

157. Crous, P. W., U. Braun, K. Schubert, and J. Z. Groenewald. 2007. Delimiting Cladosporium from morphologically similar genera. Stud. Mycol. 58: 33-56.

158. Crous, P. W., B. Slippers, M. J. Wingfield, J. Rheeder, W. F. Marasas, A. J. Philips, A. Alves, T. Burgess, P. Barber, and J. Z. Groenewald. 2006. Phylogenetic lineages in the Botryosphaeriaceae. Stud. Mycol. 55:235-253.

159. Crous, P. W., W. Gams, M. J. Wingfield, and P. S. van Wyk. 1996. Phae oacremonium gen. nov. associated with wilt and decline disease of woody hosts and human infections. Mycologia 88:786-796.

160. Cuenca-Estrella, M., A. Alastruey-Izquierdo, L. Alcazar-Fuoli, L. BernalMartinez, A. Gomez-Lopez, M. J. Buitrago, E. Mellado, and J. L. Rodriguez-Tudela. 2008. In vitro activities of 35 double combinations of antifungal agents against Scedosporium apiospermum and Scedosporium prolificans. Antimicrob. Agents Chemother. 52:1136-1139.

161. Cuenca-Estrella, M., A. Gomez-Lopez, E. Mellado, M. J. Buitrago, A. Monzon, and J. L. Rodriguez-Tudela. 2006. Head-to-head comparison of the activities of currently available antifungal agents against 3,378 Spanish clinical isolates of yeasts and filamentous fungi. Antimicrob. Agents Chemother. 50:917-921.

162. Cuenca-Estrella, M., A. Gomez-Lopez, E. Mellado, G. Garcia-Effron, A. Monzon, and J. L. Rodriguez-Tudela. 2005. In vitro activity of ravuconazole against 923 clinical isolates of nondermatophyte filamentous fungi. Antimicrob. Agents Chemother. 49:5136-5138.

163. Cuenca-Estrella, M., B. Ruiz-Diez, J. V. Martinez-Suarez, A. Monzon, and J. L. Rodriguez-Tudela. 1999. Comparative in-vitro activity of voriconazole (UK-109,496) and six other antifungal agents against clinical isolates of Scedosporium prolificans and Scedosporium apiospermum. J. Antimicrob. Chemother. 43:149-151.

164. Cuetara, M. S., A. Alhambra, M. D. Moragues, E. Gonzalez-Elorza, J. Ponton, and A. del Palacio. 2009. Detection of (1,3)-beta-D-glucan as an adjunct to diagnosis in a mixed population with uncommon proven invasive fungal diseases or with an unusual clinical presentation. Clin. Vaccine Immunol. 16:423-426.

165. Dadachova, E., and A. Casadevall. 2008. Ionizing radiation: how fungi cope, adapt, and exploit with the help of melanin. Curr. Opin. Microbiol. 11:525 531.

166. Dalton, P. A., W. J. Munckhof, and D. W. Walters. 2006. Scedosporium prolificans: an uncommon cause of septic arthritis. Aust. N. Z. J. Surg. 76:661-663

167. Dan, M., O. Yossepowitch, D. Hendel, O. Shwartz, and D. A. Sutton. 2006. Phialemonium curvatum arthritis of the knee following intra-articular injection of a corticosteroid. Med. Mycol. 44:571-574.

168. Dar, L., S. Singh, U. Banerjee, A. Verma, and R. Bhatia. 1993. Brain abscess due to Xylohypha bantiana. Indian J. Med. Microbiol. 11:148-150.

169. Dastur, H. M., A. P. Chaukar, and M. D. Rebello. 1966. Cerebral chromoblastomycosis due to Cladosporium trichoides (bantianum). I. A review and case report. Neurol. India 14:1-5.

170. Deb, S., A. K. Khan, B. Debasish, and B. Subroto. 2005. Intracranial necrotizing granuloma caused by Cladophialophora bantiana. Neurol. India 53:335-336.

171. De Battle, J., M. Motje, R. Balanza, R. Guardia, and R. Ortiz. 2000. 
Disseminated infection caused by Scedosporium prolificans in a patient with multilineal leukemia. J. Clin. Microbiol. 38:1694-1695.

172. De Hoog, G. S., D. Adelmann, A. O. Ahmed, and A. van Belkum. 2004 Phylogeny and typification of Madurella mycetomatis, with a comparison of other agents of eumycetoma. Mycoses 47:121-130.

173. De Hoog, G. S., and N. A. Yurlova. 1994. Conidiogenesis, nutritional physiology and taxonomy of Aureobasidium and Hormonema. Antonie Van Leeuwenhoek 65:41-54.

174. De Hoog, G. S., D. Attili-Angelis, V. A. Vicente, A. H. Van Den Ende, and F. Queiroz-Telles. 2004. Molecular ecology and pathogenic potential of Fonsecaea species. Med. Mycol. 42:405-416.

175. De Hoog, G. S., J. Guarro, J. Gene, and M. J. Figueras. 2000. Atlas of clinical fungi. Centraalbureau voor Schimmelcultures, Utrecht, Netherlands.

176. De Hoog, G. S., and R. Horre. 2002. Molecular taxonomy of the Alternaria and Ulocladium species from humans and their identification in the routine laboratory. Mycoses 45:259-276.

177. De Hoog, G. S., J. Guarro, J. Gené, and M. J. Fígueras. 2009. Atlas of clinical fungi, pilot version of 3rd ed., CD-ROM. Centraalbureau voor Schimmelcultures, Baarn, Netherlands.

178. De Hoog, G. S., T. Matos, M. Sudhadham, K. F. Luijsterburg, and G. Haase. 2005. Intestinal prevalence of the neurotropic black yeast Exophiala (Wangiella) dermatitidis in healthy and impaired individuals. Mycoses 48 : $142-145$.

179. De Hoog, G. S., P. Mayser, G. Haase, R. Horre, and A. M. Horrevorts. 2000 A new species, Phialophora europaea, causing superficial infections in humans. Mycoses 43:409-416.

180. De Hoog, G. S., N. Poonwan, and A. H. G. Gerrits van den Ende. 1999. Taxonomy of Exophiala spinifera and its relationship to E. jeanselmei. Stud. Mycol. 43:133-142.

181. De Hoog, G. S., A. S. Nishikaku, G. Fernandez-Zeppenfeldt, C. PadinGonzalez, E. Burger, H. Badali, N. Richard-Yegres, and A. H. van den Ende. 2007. Molecular analysis and pathogenicity of the Cladophialophora carrionii complex, with the description of a novel species. Stud. Mycol. 58:219-234

182. De Hoog, G. S., K. Takeo, E. Gottlich, K. Nishimura, and M. Miyaji. 1995. A human isolate of Exophiala (Wangiella) dermatitidis forming a catenate synanamorph that links the genera Exophiala and Cladophialophora. J. Med. Vet. Mycol. 33:355-358.

183. De Hoog, G. S., K. Takeo, S. Yoshida, E. Gottlich, K. Nishimura, and M Miyaji. 1994. Pleoanamorphic life cycle of Exophiala (Wangiella) dermatitidis. Antonie Van Leeuwenhoek 65:143-153.

184. De Hoog, G. S., V. Vicente, R. B. Caligiorne, S. Kantarcioglu, K. Tintelnot, A. H. Gerrits van den Ende, and G. Haase. 2003. Species diversity and polymorphism in the Exophiala spinifera clade containing opportunistic black yeast-like fungi. J. Clin. Microbiol. 41:4767-4778.

185. De Hoog, G. S., X. O. Weenink, and A. H. G. Gerrits van den Ende. 1999. Taxonomy of the Phialophora verrucosa complex with the description of four new species. Stud. Mycol. 43:107-142.

186. De Hoog, G. S., and A. H. G. Gerrits van den Ende. 1992. Nutritional pattern and ecophysiology of Hortae werneckii, agent of human tinea nigra. Antonie Van Leeuwenhoek 62:321-329.

187. De Hoog, G. S., and R. G. Vitale. 2007. Bipolaris, Exophiala, Scedosporium, Sporothrix, and other dematiaceous fungi, p. 1898-1917. In P. R. Murray, E. J. Baron, J. H. Jorgensen, M. L. Landry, and M. A. Pfaller (ed.), Manual of clinical microbiology, 9th ed. ASM Press, Washington, DC.

188. De Lastours, V., A. Lefort, M. Zappa, V. Dufour, N. Belmatoug, and B. Fantin. 2003. Two cases of cerebral aspergillosis successfully treated with voriconazole. Eur. J. Clin. Microbiol. Infect. Dis. 22:297-299.

189. Delfino, D., S. De Hoog, L. Polonelli, M. Benecchi, F. Fanti, S. Galatioto, G. Manti, and V. Cusumano. 2006. Survival of a neglected case of brain abscess caused by Cladophialophora bantiana. Med. Mycol. 44:651-654.

190. Delhaes, L., A. Harun, S. C. Chen, Q. Nguyen, M. Slavin, C. H. Heath, K Maszewska, C. Halliday, V. Robert, T. C. Sorrell, T. A. Auscedo Study Group, and W. Meyer. 2008. Molecular typing of Australian Scedosporium isolates showing genetic variability and numerous $S$. aurantiacum. Emerg. Infect. Dis. 14:282-290.

191. Del Palacio, A., C. Gomez-Hernando, F. Revenga, E. Carabias, A. Gonzalez, M. S. Cuetara, and E. M. Johnson. 1996. Cutaneous Alternaria alternata infection successfully treated with itraconazole. Clin. Exp. Dermatol. 21: 241-243.

192. Del Palacio, H. A., J. M. Conde-Zurita, P. S. Reyes, and N. A. Rodriguez. 1983. A case of Alternaria alternata (Fr.) Keissler infection of the knee. Clin. Exp. Dermatol. 8:641-646.

193. Del Palacio-Hernanz, A., M. K. Moore, C. K. Campbell, A. del PalacioPerez-Medel, and R. del Castillo-Cantero. 1989. Infection of the central nervous system by Rhinocladiella atrovirens in a patient with acquired immunodeficiency syndrome. J. Med. Vet. Mycol. 27:127-130.

194. De Monbrison, F., M. A. Piens, B. Ample, S. Euvrard, P. Cochat, and S. Picot. 2004. Two cases of subcutaneous phaeohyphomycosis due to $E x$ ophiala jeanselmei, in cardiac transplant and renal transplant patients. Br. J. Dermatol. 150:597-598.
195. De Moragas, J. M., G. Prats, and G. Verger. 1981. Cutaneous alternariosis treated with miconazole. Arch. Dermatol. 117:292-294.

196. Destino, L., D. A. Sutton, A. L. Helon, P. L. Havens, J. G. Thometz, R. E. Willoughby, Jr., and M. J. Chusid. 2006. Severe osteomyelitis caused by Myceliophthora thermophila after a pitchfork injury. Ann. Clin. Microbiol. Antimicrob. 5:21.

197. DeVault, G. A., Jr., S. T. Brown III, J. W. King, M. Fowler, and A. Oberle. 1985. Tenckhoff catheter obstruction resulting from invasion by Curvularia lunata in the absence of peritonitis. Am. J. Kidney Dis. 6:124-127.

198. Diaz, M., R. Puente, and M. A. Trevino. 1990. Response of long-running Alternaria alternata infection to fluconazole. Lancet 336:513.

199. Diemert, D., D. Kunimoto, C. Sand, and R. Rennie. 2001. Sputum isolation of Wangiella dermatitidis in patients with cystic fibrosis. Scand. J. Infect. Dis. 33:777-779.

200. Dixon, D. M., W. G. Merz, H. L. Elliott, and S. Macleay. 1987. Experimental central nervous system phaeohyphomycosis following intranasal inoculation of Xylohypha bantiana in cortisone-treated mice. Mycopathologia 100:145153.

201. Dixon, D. M., J. Migliozzi, C. R. Cooper, Jr., O. Solis, B. Breslin, and P. J. Szaniszlo. 1992. Melanized and non-melanized multicellular form mutants of Wangiella dermatitidis in mice: mortality and histopathology studies. Mycoses 35:17-21.

202. Dixon, D. M., and A. Polak. 1987. In vitro and in vivo drug studies with three agents of central nervous system phaeohyphomycosis. Chemotherapy 33:129-140.

203. Dixon, D. M., A. Polak, and P. J. Szaniszlo. 1987. Pathogenicity and virulence of wild-type and melanin-deficient Wangiella dermatitidis. J. Med. Vet. Mycol. 25:97-106.

204. Dixon, D. M., T. J. Walsh, W. G. Merz, and M. R. McGinnis. 1989. Infections due to Xylohypha bantiana (Cladosporium trichoides). Rev. Infect. Dis. 11:515-525.

205. Doering, T. L., J. D. Nosanchuk, W. K. Roberts, and A. Casadevall. 1999. Melanin as a potential cryptococcal defence against microbicidal proteins. Med. Mycol. 37:175-181.

206. Dooley, D. P., M. L. Beckius, B. S. Jeffery, C. K. McAllister, W. H. Radentz, A. R. Feldman, M. G. Rinaldi, S. R. Bailey, and J. H. Keeling. 1989. Phaeohyphomycotic cutaneous disease caused by Pleurophoma in a cardiac transplant patient. J. Infect. Dis. 159:503-507.

207. Drees, M., B. L. Wickes, M. Gupta, and S. Hadley. 2007. Lecythophora mutabilis prosthetic valve endocarditis in a diabetic patient. Med. Mycol. 45:463-467.

208. Drouhet, E., and B. Dupont. 1983. Laboratory and clinical assessment of ketoconazole in deep-seated mycoses. Am. J. Med. 74:30-47.

209. Duggan, J. M., M. D. Wolf, and C. A. Kauffman. 1995. Phialophora verru cosa infection in an AIDS patient. Mycoses 38:215-218.

210. Dunlop, A. A., E. D. Wright, S. A. Howlader, I. Nazrul, R. Husain, K. McClellan, and F. A. Billson. 1994. Suppurative corneal ulceration in Bangladesh. A study of 142 cases examining the microbiological diagnosis, clinical and epidemiological features of bacterial and fungal keratitis. Aust. N. Z. J. Ophthalmol. 22:105-110.

211. Dupuis, A., N. Tournier, G. Le Moal, and N. Venisse. 2009. Preparation and stability of voriconazole eye drop solution. Antimicrob. Agents Chemother. 53:798-799.

212. Duque, O. 1961. Meningo-encephalitis and brain abscess caused by Cladosporium and Fonsecaea. Am. J. Clin. Pathol. 36:505-517.

213. Durkin, S. R., T. Henderson, R. Raju, and D. Ellis. 2008. Successful treatment of phaeohyphomycotic keratitis caused by Bipolaris australiensis. Clin. Exp. Ophthalmol. 36:697-699.

214. Dutriaux, C., I. Saint-Cyr, N. Desbois, D. Cales-Quist, A. Diedhou, and A. M. Boisseau-Garsaud. 2005. Subcutaneous phaeohyphomycosis due to Exophiala spinifera in a renal transplant recipient. Ann. Dermatol. Venereol. 132:259-262.

215. Ebright, J. R., P. H. Chandrasekar, S. Marks, M. R. Fairfax, A. Aneziokoro, and M. R. McGinnis. 1999. Invasive sinusitis and cerebritis due to Curvularia clavata in an immunocompetent adult. Clin. Infect. Dis. 28:687-689.

216. El-Ani, A. S. 1966. A new species of Leptosphaeria, an etiologic agent of mycetoma. Mycologia 58:406-411.

217. El-Ani, A. S., and M. A. Gordon. 1965. The ascospore sheath and taxonomy of Leptosphaeria senegalensis. Mycologia 57:275-278.

218. Elewski, B. E. 1996. Onychomycosis caused by Scytalidium dimidiatum. J. Am. Acad. Dermatol. 35:336-338.

219. Elinav, H., U. Izhar, S. Benenson, D. Admon, C. Hidalgo-Grass, I. Polacheck, and M. Korem. 2009. Invasive Scytalidium dimidiatum infection in an immunocompetent adult. J. Clin. Microbiol. 47:1259-1263.

220. Ellis, M. B. 1971. Dematiaceous hyphomycetes. Commonwealth Mycological Institute, Kew, United Kingdom.

221. Ellis, M. B. 1976. More dematiaceous hyphomycetes. Commonwealth Mycological Institute, Kew, United Kingdom.

222. Emmens, R. K., D. Richardson, W. Thomas, S. Hunter, R. A. Hennigar, J. R. Wingard, and F. S. Nolte. 1996. Necrotizing cerebritis in an allogeneic bone marrow transplant recipient due to Cladophialophora bantiana. J. Clin. Microbiol. 34:1330-1332. 
223. Erwin, G. E., and J. E. Fitzgerald. 2007. Case report: allergic bronchopulmonary aspergillosis and allergic fungal sinusitis successfully treated with voriconazole. J. Asthma 44:891-895.

224. Espinel-Ingroff, A. 1998. Comparison of In vitro activities of the new triazole SCH56592 and the echinocandins MK-0991 (L-743,872) and LY303366 against opportunistic filamentous and dimorphic fungi and yeasts. J. Clin. Microbiol. 36:2950-2956.

225. Espinel-Ingroff, A. 2003. In vitro antifungal activities of anidulafungin and micafungin, licensed agents and the investigational triazole posaconazole as determined by NCCLS methods for 12,052 fungal isolates: review of the literature. Rev. Iberoam. Micol. 20:121-136.

226. Espinel-Ingroff, A. 2001. In vitro fungicidal activities of voriconazole, itraconazole, and amphotericin $\mathrm{B}$ against opportunistic moniliaceous and dematiaceous fungi. J. Clin. Microbiol. 39:954-958.

227. Espinel-Ingroff, A., K. Boyle, and D. J. Sheehan. 2001. In vitro antifunga activities of voriconazole and reference agents as determined by NCCLS methods: review of the literature. Mycopathologia 150:101-115.

228. Espinel-Ingroff, A., E. Johnson, H. Hockey, and P. Troke. 2008. Activities of voriconazole, itraconazole and amphotericin B in vitro against 590 moulds from 323 patients in the voriconazole phase III clinical studies. J. Antimicrob. Chemother. 61:616-620.

229. Esterre, P., A. Andriantsimahavandy, E. R. Ramarcel, and J. L. Pecarrere. 1996. Forty years of chromoblastomycosis in Madagascar: a review. Am. J. Trop. Med. Hyg. 55:45-47.

230. Estes, S. A., W. G. Merz, and L. G. Maxwell. 1977. Primary cutaneous phaeohyphomycosis caused by Drechslera spicifera. Arch. Dermatol. 113: 813-815.

231. European Economic Community. 2000. Directive 2000/54/EC, p. 19. In Official journal of the European Communities. Office for Official Publications of the European Communities, Luxembourg.

232. Fader, R. C., and M. R. McGinnis. 1988. Infections caused by dematiaceous fungi: chromoblastomycosis and phaeohyphomycosis. Infect. Dis. Clin. N. Am. 2:925-938.

233. Fan, Y. M., W. M. Huang, S. F. Li, G. F. Wu, W. Li, and R. Y. Chen. 2009. Cutaneous phaeohyphomycosis of foot caused by Curvularia clavata. Mycoses 52:544-546.

234. Farina, C., A. Gamba, R. Tambini, H. Beguin, and J. L. Trouillet. 1998 Fatal aortic Myceliophthora thermophila infection in a patient affected by cystic medial necrosis. Med. Mycol. 36:113-118.

235. Farina, C., E. Gotti, D. Mouniee, P. Boiron, and A. Goglio. 2007. Phaeoacremonium parasiticum subcutaneous infection in a kidney-transplanted patient successfully treated by surgery. Transpl. Infect. Dis. 9:253-255.

236. Farina, C., E. Gotti, A. Parma, L. Naldi, and A. Goglio. 2007. Pheohyphomycotic soft tissue disease caused by Alternaria alternata in a kidney transplant patient: a case report and literature review. Transplant. Proc. 39: 1655-1659.

237. Feldman, D. L., E. Fitzpatrick, O. Schaening, and L. I. Lutwick. 1995 Nosocomial phaeomycotic cyst of the hand. Ann. Plastic Surg. 35:113-115.

238. Feltkamp, M. C., M. J. Kersten, J. van der Lelie, J. D. Burggraaf, G. S. de Hoog, and E. J. Kuijper. 1997. Fatal Scedosporium prolificans infection in a leukemic patient. Eur. J. Clin. Microbiol. Infect. Dis. 16:460-464.

239. Ferguson, B. J. 2000. Definitions of fungal rhinosinusitis. Otolaryngol. Clin N. Am. 33:227-235.

240. Ferguson, B. J., L. Barnes, J. M. Bernstein, D. Brown, C. E. Clark III, P. R Cook, W. S. DeWitt, S. M. Graham, B. Gordon, A. R. Javer, J. H. Krouse, F. A. Kuhn, H. L. Levine, S. C. Manning, B. F. Marple, A. H. Morgan, J. D. Osguthorpe, D. Skedros, B. M. Rains III, H. H. Ramadan, J. E. Terrell, and A. J. Yonkers. 2000. Geographic variation in allergic fungal rhinosinusitis. Otolaryngol. Clin. N. Am. 33:441-449.

241. Fernandez, M., D. E. Noyola, S. N. Rossmann, and M. S. Edwards. 1999 Cutaneous phaeohyphomycosis caused by Curvularia lunata and a review of Curvularia infections in pediatrics. Pediatr. Infect. Dis. J. 18:727-731.

242. Ferraro, F. A., and M. A. Morgan. 1989. A case of disseminated Phialophora parasitica infection. Arch. Pathol. Lab. Med. 113:1379-1381.

243. Fica, A., M. C. Diaz, M. Luppi, R. Olivares, L. Saez, M. Baboor, and P. Vasquez. 2003. Unsuccessful treatment with voriconazole of a brain abscess due to Cladophialophora bantiana. Scand. J. Infect. Dis. 35:892-893.

244. Filizzola, M. J., F. Martinez, and S. J. Rauf. 2003. Phaeohyphomycosis of the central nervous system in immunocompetent hosts: report of a case and review of the literature. Int. J. Infect. Dis. 7:282-286.

245. Fincher, R. M., J. F. Fisher, A. A. Padhye, L. Ajello, and J. C. Steele, Jr. 1988. Subcutaneous phaeohyphomycotic abscess caused by Phialophora parasitica in a renal allograft recipient. J. Med. Vet. Mycol. 26:311-314.

246. Flanagan, K. L., and A. D. Bryceson. 1997. Disseminated infection due to Bipolaris australiensis in a young immunocompetent man: case report and review. Clin. Infect. Dis. 25:311-313.

247. Florcruz, N. V., and I. Peczon, Jr. 2008. Medical interventions for fungal keratitis. Cochrane Database Syst. Rev. 23:CD004241.

248. Flynn, B. J., P. P. Bourbeau, P. J. Cera, L. M. Scicchitano, R. L. Jordan, and W. T. Yap. 1999. Phaeohyphomycosis of the epididymis caused by Exophiala jeanselmei. J. Urol. 162:492-493.
249. Fogarty, R. V., and J. M. Tobin. 1996. Fungal melanins and their interactions with metals. Enzyme Microb. Technol. 19:311-317.

250. Fothergill, A. W., M. G. Rinaldi, and D. A. Sutton. 2009. Antifungal susceptibility testing of Exophiala spp.: a head-to-head comparison of amphotericin B, itraconazole, posaconazole and voriconazole. Med. Mycol. 47:41-43.

251. Foulet, F., C. Duvoux, C. de Bievre, C. Hezode, and S. Bretagne. 1999. Cutaneous phaeohyphomycosis caused by Veronaea bothryosa in a liver transplant recipient successfully treated with itraconazole. Clin. Infect. Dis. 29:689-690.

252. Francis, P., and T. J. Walsh. 1992. Evolving role of flucytosine in immunocompromised patients: new insights into safety, pharmacokinetics, and antifungal therapy. Clin. Infect. Dis. 15:1003-1018.

253. Franzen, A. J., M. M. Cunha, K. Miranda, J. Hentschel, H. Plattner, M. B. da Silva, C. G. Salgado, W. de Souza, and S. Rozental. 2008. Ultrastructural characterization of melanosomes of the human pathogenic fungus Fonsecaea pedrosoi. J. Struct. Biol. 162:75-84.

254. Freitas, A., D. B. Pedral-Sampaio, N. L. Espinheira, E. Daltro, J. Sampaio, P. Athanasio, C. Lacaz, and R. Badaro. 1997. Cladophialophora bantiana (previously Cladosporium trichoides): first report of a case in Brazil. Braz. J. Infect. Dis. 1:313-316.

255. Friedman, A. D., J. M. Campos, L. B. Rorke, D. A. Bruce, and A. M. Arbeter. 1981. Fatal recurrent Curvularia brain abscess. J. Pediatr. 99:413415.

256. Fukushima, N., K. Mannen, S. Okamoto, T. Shinogi, K. Nishimoto, and E. Sueoka. 2005. Disseminated Ochroconis gallopavum infection in a chronic lymphocytic leukemia: a case report and review of the literature on hematological malignancies. Intern. Med. 44:879-882.

257. Fukushiro, R. 1983. Chromomycosis in Japan. Int. J. Dermatol. 22:221-229.

258. Fukushiro, R., S. Udagawa, Y. Kawashima, and Y. Kawamura. 1986. Subcutaneous abscesses caused by Ochroconis gallopavum. J. Med. Vet. Mycol. 24:175-182.

259. Fung-Tomc, J. C., E. Huczko, B. Minassian, and D. P. Bonner. 1998. In vitro activity of a new oral triazole, BMS-207147 (ER-30346). Antimicrob. Agents Chemother. 42:313-318.

260. Fuste, F. J., L. Ajello, R. Threlkeld, and J. E. Henry, Jr. 1973. Drechslera hawaiiensis: causative agent of a fatal fungal meningo-encephalitis. Sabouraudia 11:59-63.

261. Gadallah, M. F., R. White, M. A. el Shahawy, F. Abreo, A. Oberle, and J. Work. 1995. Peritoneal dialysis complicated by Bipolaris hawaiiensis peritonitis: successful therapy with catheter removal and oral itraconazol without the use of amphotericin-B. Am. J. Nephrol. 15:348-352.

262. Gallelli, B., M. Viviani, M. Nebuloni, A. V. Marzano, C. Pozzi, P. Messa, and G. B. Fogazzi. 2006. Skin infection due to Alternaria species in kidney allograft recipients: report of a new case and review of the literature. J. Nephrol. 19:668-672.

263. Gams, W. 2000. Phialophora and some similar morphologically little-differ entiated anamorphs of divergent ascomycetes. Stud. Mycol. 45:187-199.

264. Gams, W., and M. R. McGinnis. 1983. Phialemonium, a new anamorph genus intermediate between Phialophora and Acremonium. Mycologia 75: 977-987.

265. Garau, J., R. D. Diamond, L. B. Lagrotteria, and S. A. Kabins. 1977. Alternaria osteomyelitis. Ann. Intern. Med. 86:747-748.

266. Garcia-Effron, G., A. Gomez-Lopez, E. Mellado, A. Monzon, J. L. Rodriguez-Tudela, and M. Cuenca-Estrella. 2004. In vitro activity of terbinafine against medically important non-dermatophyte species of filamentous fungi. J. Antimicrob. Chemother. 53:1086-1089.

267. Garcia-Ruiz, J. C., E. Amutio, I. Hernandez, C. Alvarez, F. Floristan, I. Zuazua, A. Alvarez-Blanco, and J. Ponton. 1998. Clinical resolution of Scedosporium prolificans pneumonia associated with treatment with liposomal amphotericin B in a patient with acute leukemia. Rev. Iberoam. Micol. 15:158-159.

268. Garg, N., I. B. Devi, G. V. Vajramani, S. Nagarathna, S. Sampath, B. A. Chandramouli, A. Chandramuki, and S. K. Shankar. 2007. Central nervous system cladosporiosis: an account of ten culture-proven cases. Neurol. India 55:282-288.

269. Garg, P., U. Gopinathan, K. Choudhary, and G. N. Rao. 2000. Keratomycosis: clinical and microbiologic experience with dematiaceous fungi. Ophthalmology 107:574-580.

270. Garg, P., G. K. Vemuganti, S. Chatarjee, U. Gopinathan, and G. N. Rao. 2004. Pigmented plaque presentation of dematiaceous fungal keratitis: a clinicopathologic correlation. Cornea 23:571-576.

271. Garrison, A. P., G. W. Procop, V. Vincek, J. Moon, M. I. Morris, S. Doblecki-Lewis, T. J. Cleary, D. Brust, and I. Rosa-Cunha. 2008. A case of subcutaneous Mycoleptodiscus indicus infection in a liver transplant recipient successfully treated with antifungal therapy. Transpl. Infect. Dis. 10: 218-220.

272. Garzoni, C., L. Markham, P. Bijlenga, and J. Garbino. 2008. Cladophialophora bantiana: a rare cause of fungal brain abscess. Clinical aspects and new therapeutic options. Med. Mycol. 46:481-486.

273. Gavin, P. J., D. A. Sutton, and B. Z. Katz. 2002. Fatal endocarditis in a 
neonate caused by the dematiaceous fungus Phialemonium obovatum: case report and review of the literature. J. Clin. Microbiol. 40:2207-2212.

274. Geis, P. A., M. H. Wheeler, and P. J. Szaniszlo. 1984. Pentaketide metabolites of melanin synthesis in the dematiaceous fungus Wangiella dermatitidis. Arch. Microbiol. 137:324-328.

275. Gene, J., A. Azon-Masoliver, J. Guarro, F. Ballester, I. Pujol, M. Llovera, and C. Ferrer. 1995. Cutaneous phaeohyphomycosis caused by Alternaria longipes in an immunosuppressed patient. J. Clin. Microbiol. 33:2774-2776.

276. George, I. A., M. S. Mathews, R. Karthik, L. John, A. Sundar, O. C. Abraham, and V. Joseph. 2008. Fatal cerebral abscess caused by Cladophialophora bantiana. J. Assoc. Physicians India 56:470-472.

277. Gerdsen, R., M. Uerlich, G. S. De Hoog, T. Bieber, and R. Horre. 2001. Sporotrichoid phaeohyphomycosis due to Alternaria infectoria. Br. J. Dermatol. 145:484-486.

278. Gergen, P. J., P. C. Turkeltaub, and M. G. Kovar. 1987. The prevalence of allergic skin test reactivity to eight common aeroallergens in the U.S. population: results from the second National Health and Nutrition Examination Survey. J. Allergy Clin. Immunol. 80:669-679.

279. Gerrits van den Ende, A. H. G., and G. S. de Hoog. 1999. Variability and molecular diagnostics of the neurotropic species Cladophialophora bantiana. Stud. Mycol. 43:151-162.

280. Gilaberte, M., R. Bartralot, J. M. Torres, F. S. Reus, V. Rodriguez, A Alomar, and R. M. Pujol. 2005. Cutaneous alternariosis in transplant recipients: clinicopathologic review of 9 cases. J. Am. Acad. Dermatol. 52: 653-659.

281. Gilgado, F., J. Cano, J. Gene, and J. Guarro. 2005. Molecular phylogeny of the Pseudallescheria boydii species complex: proposal of two new species. J. Clin. Microbiol. 43:4930-4942.

282. Gilgado, F., J. Cano, J. Gene, D. A. Sutton, and J. Guarro. 2008. Molecular and phenotypic data supporting distinct species statuses for Scedosporium apiospermum and Pseudallescheria boydii and the proposed new species Scedosporium dehoogii. J. Clin. Microbiol. 46:766-771.

283. Gilgado, F., J. Gene, J. Cano, and J. Guarro. 2010. Heterothallism in Scedosporium apiospermum and description of its teleomorph Pseudallescheria apiosperma sp. nov. Med. Mycol. 48:122-128.

284. Gil-Lamaignere, C., A. Maloukou, J. L. Rodriguez-Tudela, and E. Roilides. 2001. Human phagocytic cell responses to Scedosporium prolificans. Med. Mycol. 39:169-175.

285. Gil-Lamaignere, C., E. Roilides, J. Mosquera, A. Maloukou, and T. J. Walsh. 2002. Antifungal triazoles and polymorphonuclear leukocytes synergize to cause increased hyphal damage to Scedosporium prolificans and Scedosporium apiospermum. Antimicrob. Agents Chemother. 46 2234-2237.

286. Gilmour, T. K., E. Rytina, P. B. O'Connell, and J. C. Sterling. 2001 Cutaneous alternariosis in a cardiac transplant recipient. Australas. J. Dermatol. 42:46-49.

287. Ginarte, M., M. Pereiro, Jr., V. Fernandez-Redondo, and J. Toribio. 1996 Plantar infection by Scopulariopsis brevicaulis. Dermatology 193:149-151.

288. Girardi, L. S., R. Malowitz, G. T. Tortora, and E. D. Spitzer. 1993. Aureobasidium pullulans septicemia. Clin. Infect. Dis. 16:338-339.

289. Godfrey, J. 1846. Diseases of the foot not hitherto described. Lancet i:593594.

290. Goel, A., A. Satoskar, A. P. Desai, and S. K. Pandya. 1992. Brain abscess caused by Cladosporium trichoides. Br. J. Neurosurg. 6:591-593.

291. Gold, W. L., H. Vellend, I. E. Salit, I. Campbell, R. Summerbell, M. Rinaldi, and A. E. Simor. 1994. Successful treatment of systemic and local infections due to Exophiala species. Clin. Infect. Dis. 19:339-341.

292. Gomez, B. L., and J. D. Nosanchuk. 2003. Melanin and fungi. Curr. Opin. Infect. Dis. 16:91-96.

293. Gomez, B. L., J. D. Nosanchuk, S. Diez, S. Youngchim, P. Aisen, L. E. Cano, A. Restrepo, A. Casadevall, and A. J. Hamilton. 2001. Detection of melanin-like pigments in the dimorphic fungal pathogen Paracoccidioides brasiliensis in vitro and during infection. Infect. Immun. 69:5760-5767.

294. Gonzalez, G. M. 2009. In vitro activities of isavuconazole against opportunistic filamentous and dimorphic fungi. Med. Mycol. 47:71-76.

295. Gonzalez, M. S., B. Alfonso, D. Seckinger, A. A. Padhye, and L. Ajello. 1984 Subcutaneous phaeohyphomycosis caused by Cladosporium devriesii, sp. nov. Sabouraudia 22:427-432.

296. Gonzalez-Lopez, M. A., R. Salesa, M. C. Gonzalez-Vela, H. FernandezLlaca, J. F. Val-Bernal, and J. Cano. 2007. Subcutaneous phaeohyphomycosis caused by Exophiala oligosperma in a renal transplant recipient. Br. J. Dermatol. 156:762-764.

297. Goodpasture, H. C., T. Carlson, B. Ellis, and G. Randall. 1983. Alternaria osteomyelitis. Evidence of specific immunologic tolerance. Arch. Pathol. Lab. Med. 107:528-530.

298. Gopinathan, U., P. Garg, M. Fernandes, S. Sharma, S. Athmanathan, and G. N. Rao. 2002. The epidemiological features and laboratory results of fungal keratitis: a 10-year review at a referral eye care center in South India. Cornea 21:555-559.

299. Gosbell, I. B., V. Toumasatos, J. Yong, R. S. Kuo, D. H. Ellis, and R. C. Perrie. 2003. Cure of orthopaedic infection with Scedosporium prolificans using voriconazole plus terbinafine, without the need for radical surgery. Mycoses 46:233-236.

300. Gottlich, E., W. van der Lubbe, B. Lange, S. Fiedler, I. Melchert, M. Reifenrath, H. C. Flemming, and S. de Hoog. 2002. Fungal flora in groundwater-derived public drinking water. Int. J. Hyg. Environ. Health 205:269279.

301. Graybill, J. R., L. K. Najvar, E. Johnson, R. Bocanegra, and D. Loebenberg. 2004. Posaconazole therapy of disseminated phaeohyphomycosis in a murine model. Antimicrob. Agents Chemother. 48:2288-2291.

302. Greig, J., M. Harkness, P. Taylor, C. Hashmi, S. Liang, and J. Kwan. 2003. Peritonitis due to the dermatiaceous mold Exophiala dermatitidis complicating continuous ambulatory peritoneal dialysis. Clin. Microbiol. Infect. 9:713-715.

303. Greig, J. R., M. A. Khan, N. S. Hopkinson, B. G. Marshall, P. O. Wilson, and S. U. Rahman. 2001. Pulmonary infection with Scedosporium prolificans in an immunocompetent individual. J. Infect. 43:15-17.

304. Grenouillet, F., F. Botterel, J. Crouzet, F. Larosa, Y. Hicheri, J. M. Forel, P. Helias, S. Ranque, and L. Delhaes. 2009. Scedosporium prolificans: an emerging pathogen in France? Med. Mycol. 47:343-350.

305. Guarner, J., C. Del Rio, P. Williams, and J. E. McGowan, Jr. 1989. Fungal peritonitis caused by Curvularia lunata in a patient undergoing peritoneal dialysis. Am. J. Med. Sci. 298:320-323.

306. Guarro, J., H. C. Gugnani, N. Sood, R. Batra, E. Mayayo, J. Gene, and S. Kakkar. 2008. Subcutaneous phaeohyphomycosis caused by Wallemia sebi in an immunocompetent host. J. Clin. Microbiol. 46:1129-1131.

307. Guarro, J., E. Mayayo, J. Tapiol, C. Aguilar, and J. Cano. 1999. Microsphaeropsis olivacea as an etiological agent of human skin infection. Med. Mycol. 37:133-137.

308. Guarro, J., M. Nucci, T. Akiti, J. Gene, J. Cano, M. D. Barreiro, and C Aguilar. 1999. Phialemonium fungemia: two documented nosocomial cases. J. Clin. Microbiol. 37:2493-2497.

309. Guarro, J., A. M. Silvestre, Jr., G. Verkley, J. Cano, O. F. Gompertz, J. Gene, M. M. Ogawa, J. Tomimori-Yamashita, S. P. Teixeira, and F. A. de Almeida. 2006. Limitations of DNA sequencing for diagnosis of a mixed infection by two fungi, Phaeoacremonium venezuelense and a Plectophomella sp., in a transplant recipient. J. Clin. Microbiol. 44:4279-4282.

310. Gubbins, P. O., and S. Heldenbrand. 2010. Clinically relevant drug interactions of current antifungal agents. Mycoses 53:95-113.

311. Gueho, E., A. Bonnefoy, J. Luboinski, J. C. Petit, and G. S. De Hoog. 1989 Subcutaneous granuloma caused by Phialophora richardsiae: case report and review of the literature. Mycoses 32:219-223.

312. Guerrero, A., P. Torres, M. T. Duran, B. Ruiz-Diez, M. Rosales, and J. L. Rodriguez-Tudela. 2001. Airborne outbreak of nosocomial Scedosporium prolificans infection. Lancet 357:1267-1268.

313. Gugnani, H. C., V. Ramesh, N. Sood, J. Guarro, H. Moin Ul, A. PaliwalJoshi, and B. Singh. 2006. Cutaneous phaeohyphomycosis caused by Cla dosporium oxysporum and its treatment with potassium iodide. Med. Mycol. 44:285-288.

314. Guppy, K. H., C. Thomas, K. Thomas, and D. Anderson. 1998. Cerebral fungal infections in the immunocompromised host: a literature review and a new pathogen-Chaetomium atrobrunneum: case report. Neurosurgery 43:1463-1469.

315. Gupta, A. K., and Y. Kohli. 2003. In vitro susceptibility testing of ciclopirox, terbinafine, ketoconazole and itraconazole against dermatophytes and nondermatophytes, and in vitro evaluation of combination antifungal activity. Br. J. Dermatol. 149:296-305.

316. Gupta, A. K., J. E. Ryder, R. Baran, and R. C. Summerbell. 2003. Nondermatophyte onychomycosis. Dermatol. Clin. 21:257-268.

317. Gupta, A. K., P. R. Taborda, and A. D. Sanzovo. 2002. Alternate week and combination itraconazole and terbinafine therapy for chromoblastomycosis caused by Fonsecaea pedrosoi in Brazil. Med. Mycol. 40:529-534.

318. Gupta, G., A. D. Burden, G. S. Shankland, M. E. Fallowfield, and M. D. Richardson. 1997. Tinea nigra secondary to Exophiala werneckii responding to itraconazole. Br. J. Dermatol. 137:483-484.

319. Gupta, S. K., K. S. Manjunath-Prasad, B. S. Sharma, V. K. Khosla, V. K. Kak, M. Minz, and V. K. Sakhuja. 1997. Brain abscess in renal transplant recipients: report of three cases. Surg. Neurol. 48:284-287.

320. Haase, G., H. Skopnik, T. Groten, G. Kusenbach, and H. G. Posselt. 1991. Long-term fungal cultures from sputum of patients with cystic fibrosis. Mycoses 34:373-376.

321. Halaby, T., H. Boots, A. Vermeulen, A. Van Der Ven, H. Beguin, H. Van Hooff, and J. Jacobs. 2001. Phaeohyphomycosis caused by Alternaria infectoria in a renal transplant patient. J. Clin. Microbiol. 39:1952-1955.

322. Haldane, D. J., and E. Robart. 1990. A comparison of calcofluor white, potassium hydroxide, and culture for the laboratory diagnosis of superficial fungal infection. Diagn. Microbiol. Infect. Dis. 13:337-339.

323. Halwig, J. M., D. A. Brueske, P. A. Greenberger, R. B. Dreisin, and H. M. Sommers. 1985. Allergic bronchopulmonary curvulariosis. Am. Rev. Resp. Dis. 132:186-188

324. Hamilton, A. J., and B. L. Gomez. 2002. Melanins in fungal pathogens. J. Med. Microbiol. 51:189-191.

325. Harkness, B., D. Andresen, A. Kesson, and D. Isaacs. 2009. Infections 
following lawnmower and farm machinery-related injuries in children. J. Paediatr. Child Health 45:525-528.

326. Harrington, B. J., and G. J. Hageage. 2003. Calcofluor white: a review of its uses and applications in clinical mycology and parasitology. Lab. Med. 34:361-367.

327. Harris, J. E., D. A. Sutton, A. Rubin, B. Wickes, G. S. De Hoog, and C. Kovarik. 2009. Exophiala spinifera as a cause of cutaneous phaeohyphomycosis: case study and review of the literature. Med. Mycol. 47:87-93.

328. Harrison, D. K., S. Moser, and C. A. Palmer. 2008. Central nervous system infections in transplant recipients by Cladophialophora bantiana. South. Med. J. 101:292-296.

329. Hart, A. P., D. A. Sutton, P. J. McFeeley, and M. Kornfeld. 2001. Cerebral phaeohyphomycosis caused by a dematiaceous Scopulariopsis species. Clin. Neuropathol. 20:224-228.

330. Harun, A., H. Perdomo, F. Gilgado, S. C. Chen, J. Cano, J. Guarro, and W. Meyer. 2009. Genotyping of Scedosporium species: a review of molecular approaches. Med. Mycol. 47:406-414.

331. Haselwandter, K., and M. R. Ebner. 1994. Microorganisms surviving for 5300 years. FEMS Microbiol. Lett. 116:189-193.

332. Hauck, E. F., M. McGinnis, and H. J. Nauta. 2008. Cerebral phaeohyphomycosis mimics high-grade astrocytoma. J. Clin. Neurosci. 15:1061-1066.

333. Hay, R. J. 1999. Therapeutic potential of terbinafine in subcutaneous and systemic mycoses. Br. J. Dermatol. 141:36-40.

334. Hayakawa, M., E. E. Ghosn, M. da Gloria Teixeria de Sousa, K. S. Ferreira, and S. R. Almeida. 2006. Phagocytosis, production of nitric oxide and pro-inflammatory cytokines by macrophages in the presence of dematiaceous fungi that cause chromoblastomycosis. Scand. J. Immunol. 64:382387.

335. Heath, C. H., J. L. Lendrum, B. L. Wetherall, S. L. Wesselingh, and D. L. Gordon. 1997. Phaeoacremonium parasiticum infective endocarditis following liver transplantation. Clin. Infect. Dis. 25:1251-1252.

336. Heath, C. H., M. A. Slavin, T. C. Sorrell, R. Handke, A. Harun, M. Phillips, Q. Nguyen, L. Delhaes, D. Ellis, W. Meyer, and S. C. Chen. 2009. Population-based surveillance for scedosporiosis in Australia: epidemiology, disease manifestations and emergence of Scedosporium aurantiacum infection. Clin. Microbiol. Infect. 15:689-693.

337. Heney, C., E. Song, A. Kellen, F. Raal, S. D. Miller, and V. Davis. 1989 Cerebral phaeohyphomycosis caused by Xylohypha bantiana. Eur. J. Clin. Microbiol. Infect. Dis. 8:984-988.

338. Herbrecht, R. 2004. Posaconazole: a potent, extended-spectrum triazole anti-fungal for the treatment of serious fungal infections. Int. J. Clin. Pract 58:612-624.

339. Herbrecht, R., S. Natarajan-Ame, Y. Nivoix, and V. Letscher-Bru. 2003. The lipid formulations of amphotericin B. Expert Opin. Pharmacother. 4:1277-1287.

340. Hibbett, D. S., M. Binder, J. F. Bischoff, M. Blackwell, P. F. Cannon, O. E Eriksson, S. Huhndorf, T. James, P. M. Kirk, R. Lucking, H. Thorsten Lumbsch, F. Lutzoni, P. B. Matheny, D. J. McLaughlin, M. J. Powell, S. Redhead, C. L. Schoch, J. W. Spatafora, J. A. Stalpers, R. Vilgalys, M. C. Aime, A. Aptroot, R. Bauer, D. Begerow, G. L. Benny, L. A. Castlebury, P. W. Crous, Y. C. Dai, W. Gams, D. M. Geiser, G. W. Griffith, C. Gueidan, D. L. Hawksworth, G. Hestmark, K. Hosaka, R. A. Humber, K. D. Hyde, J. E. Ironside, U. Koljalg, C. P. Kurtzman, K. H. Larsson, R. Lichtwardt, J. Longcore, J. Miadlikowska, A. Miller, J. M. Moncalvo, S. Mozley-Standridge, F. Oberwinkler, E. Parmasto, V. Reeb, J. D. Rogers, C. Roux, L. Ryvarden, J. P. Sampaio, A. Schussler, J. Sugiyama, R. G. Thorn, L. Tibell, W. A. Untereiner, C. Walker, Z. Wang, A. Weir, M. Weiss, M. M. White, K Winka, Y. J. Yao, and N. Zhang. 2007. A higher-level phylogenetic classification of the fungi. Mycol. Res. 111:509-547.

341. Hipolito, E., E. Faria, A. F. Alves, G. S. de Hoog, J. Anjos, T. Goncalves, P. V. Morais, and H. Estevao. 2009. Alternaria infectoria brain abscess in a child with chronic granulomatous disease. Eur. J. Clin. Microbiol. Infect. Dis. 28:377-380.

342. Hironaga, M., K. Nakano, I. Yokoyama, and J. Kitajima. 1989. Phialophora repens, an emerging agent of subcutaneous phaeohyphomycosis in humans. J. Clin. Microbiol. 27:394-399.

343. Hironaga, M., and S. Watanabe. 1980. Cerebral phaeohyphomycosis caused by Cladosporium bantianum: a case in a female who had cutaneous alternariosis in her childhood. Sabouraudia 18:229-235.

344. Hirsch, B. E., B. F. Farber, J. F. Shapiro, and S. Kennelly. 1996. Successful treatment of Aureobasidium pullulans prosthetic hip infection. Infect. Dis. Clin. Pract. 5:205-207.

345. Hiruma, M., A. Kawada, H. Ohata, Y. Ohnishi, H. Takahashi, M. Yamazaki, A. Ishibashi, K. Hatsuse, M. Kakihara, and M. Yoshida. 1993. Systemic phaeohyphomycosis caused by Exophiala dermatitidis. Mycoses 36:1-7.

346. Hohl, P. E., H. P. Holley, Jr., E. Prevost, L. Ajello, and A. A. Padhye. 1983. Infections due to Wangiella dermatitidis in humans: report of the first documented case from the United States and a review of the literature. Rev Infect. Dis. 5:854-864.

347. Holker, U., J. Bend, R. Pracht, L. Tetsch, T. Muller, M. Hofer, and G. S. de
Hoog. 2004. Hortaea acidophila, a new acid-tolerant black yeast from lignite. Antonie Van Leeuwenhoek 86:287-294.

348. Hollingsworth, J. W., S. Shofer, and A. Zaas. 2007. Successful treatment of Ochroconis gallopavum infection in an immunocompetent host. Infection 35:367-369.

349. Hong, K. H., J. W. Kim, S. J. Jang, E. Yu, and E. C. Kim. 2009. Liver cirrhosis caused by Exophiala dermatitidis. J. Med. Microbiol. 58:674-677.

350. Hood, S. V., C. B. Moore, J. S. Cheesbrough, A. Mene, and D. W. Denning. 1997. Atypical eumycetoma caused by Phialophora parasitica successfully treated with itraconazole and flucytosine. Br. J. Dermatol. 136:953-956.

351. Hoppin, E. C., E. L. McCoy, and M. G. Rinaldi. 1983. Opportunistic mycotic infection caused by Chaetomium in a patient with acute leukemia. Cancer 52:555-556.

352. Hopwood, V., E. G. Evans, J. Matthews, and D. W. Denning. 1995. Scedosporium prolificans, a multi-resistant fungus, from a U.K. AIDS patient. J. Infect. 30:153-155.

353. Horré, R., and G. S. De Hoog. 1999. Primary cerebral infections by melanized fungi: a review. Stud. Mycol. 43:176-193.

354. Horré, R., G. S. de Hoog, C. Kluczny, G. Marklein, and K. P. Schaal. 1999. rDNA diversity of Ochroconis and Scolecobasidium species isolated from humans and animals. Stud. Mycol. 43:194-205.

355. Horré, R., K. P. Schaal, B. Siekmeier, B. Sterzik, G. S. de Hoog, and N. Schnitzler. 2004. Isolation of fungi, especially Exophiala dermatitidis, in patients suffering from cystic fibrosis. Respiration 71:360-366.

356. Hosseini-Yeganeh, M., and A. J. McLachlan. 2002. Physiologically based pharmacokinetic model for terbinafine in rats and humans. Antimicrob. Agents Chemother. 46:2219-2228.

357. Houser, S. M., and J. P. Corey. 2000. Allergic fungal rhinosinusitis: pathophysiology, epidemiology, and diagnosis. Otolaryngol. Clin. N. Am. 33:399409.

358. Howden, B. P., M. A. Slavin, A. P. Schwarer, and A. M. Mijch. 2003. Successful control of disseminated Scedosporium prolificans infection with combination of voriconazole and terbinafine. Eur. J. Clin. Microbiol. Infect. Dis. 22:111-113.

359. Hsu, M. M., and J. Y. Lee. 1993. Cutaneous and subcutaneous phaeohyphomycosis caused by Exserohilum rostratum. J. Am. Acad. Dermatol. 28: 340-344.

360. Huang, Y. T., S. J. Liaw, C. H. Liao, J. L. Yang, D. M. Lai, Y. C. Lee, and P. R. Hsueh. 2008. Catheter-related septicemia due to Aureobasidium pullulans. Int. J. Infect. Dis. 12:e137-139.

361. Huhndorf, S., A. Miller, and F. Fernádez. 2004. Molecular systematics of the Sordariales: the order and the family Lasiosphaeriaceaae redefined. Mycologia 2:368-387.

362. Hurley, M. A., and B. Saffran. 1997. Subcutaneous phaeohyphomycosis: overview and case report. J. Foot Ankle Surg. 36:230-235.

363. Husain, S., B. D. Alexander, P. Munoz, R. K. Avery, S. Houston, T. Pruett, R. Jacobs, E. A. Dominguez, J. G. Tollemar, K. Baumgarten, C. M. Yu, M. M. Wagener, P. Linden, S. Kusne, and N. Singh. 2003. Opportunistic mycelial fungal infections in organ transplant recipients: emerging importance of non-Aspergillus mycelial fungi. Clin. Infect. Dis. 37:221-229.

364. Husain, S., P. Munoz, G. Forrest, B. D. Alexander, J. Somani, K. Brennan, M. M. Wagener, and N. Singh. 2005. Infections due to Scedosporium apiospermum and Scedosporium prolificans in transplant recipients: clinical characteristics and impact of antifungal agent therapy on outcome. Clin. Infect. Dis. 40:89-99.

365. Hussey, S. M., R. Gander, P. Southern, and M. P. Hoang. 2005. Subcutaneous phaeohyphomycosis caused by Cladophialophora bantiana. Arch. Pathol. Lab. Med. 129:794-797.

366. Huttova, M., K. Kralinsky, J. Horn, I. Marinova, K. Iligova, J. Fric, S. Spanik, J. Filka, J. Uher, J. Kurak, and V. Krcmery, Jr. 1998. Prospective study of nosocomial fungal meningitis in children-report of 10 cases. Scand. J. Infect. Dis. 30:485-487.

367. Ibanez, P. R., J. Chacon, A. Fidalgo, J. Martin, V. Paraiso, and J. L. Munoz-Bellido. 1997. Peritonitis by Aureobasidium pullulans in continuous ambulatory peritoneal dialysis. Nephrol. Dial. Transplant. 12:1544-1545.

368. Idigoras, P., E. Perez-Trallero, L. Pineiro, J. Larruskain, M. C. LopezLopategui, N. Rodriguez, and J. M. Gonzalez. 2001. Disseminated infection and colonization by Scedosporium prolificans: a review of 18 cases, 19901999. Clin. Infect. Dis. 32:e158-e165.

369. Ikai, K., H. Tomono, and S. Watanabe. 1988. Phaeohyphomycosis caused by Phialophora richardsiae. J. Am. Acad. Dermatol. 19:478-481.

370. Ikeda, R., T. Sugita, E. S. Jacobson, and T. Shinoda. 2003. Effects of melanin upon susceptibility of Cryptococcus to antifungals. Microbiol. Immunol. 47:271-277.

371. Imwidthaya, P. 1994. Systemic fungal infections in Thailand. J. Med. Vet. Mycol. 32:395-399.

372. Inoue, Y., Y. Matsuwaki, S. H. Shin, J. U. Ponikau, and H. Kita. 2005. Nonpathogenic, environmental fungi induce activation and degranulation of human eosinophils. J. Immunol. 175:5439-5447.

373. Iwatsu, T. 1988. Cutaneous alternariosis. Arch. Dermatol. 124:1822-1825.

374. Iyer, S. A., S. S. Tuli, and R. C. Wagoner. 2006. Fungal keratitis: emerging trends and treatment outcomes. Eye Contact Lens 32:267-271. 
375. Jacobson, E. S. 2000. Pathogenic roles for fungal melanins. Clin. Microbiol Rev. 13:708-717.

376. Jacobson, E. S., and G. M. Compton. 1996. Discordant regulation of phenoloxidase and capsular polysaccharide in Cryptococcus neoformans. J. Med. Vet. Mycol. 34:289-291.

377. Jacyk, W. K. 1979. Chromomycosis due to Cladosporium carrionii treated with 5-fluorocytosine. A case report from northern Nigeria. Cutis 23:649650.

378. Janaki, C., G. Sentamilselvi, V. R. Janaki, S. Devesh, and K. Ajithados. 1999. Case report. Eumycetoma due to Curvularia lunata. Mycoses 42:345346.

379. Jay, W. M., R. W. Bradsher, B. LeMay, N. Snyderman, and E. J. Angtuaco. 1988. Ocular involvement in mycotic sinusitis caused by Bipolaris. Am. J. Ophthalmol. 105:366-370.

380. Jayakeerthi, S. R., M. Dias, S. Nagarathna, B. Anandh, A. Mahadevan, and A. Chandramuki. 2004. Brain abscess due to Cladophialophora bantiana. Indian J. Med. Microbiol. 22:193-195.

381. Jensen, J. C. 1989. Clinical pharmacokinetics of terbinafine (Lamisil). Clin. Exp. Dermatol. 14:110-113.

382. Jessup, C. J., N. S. Ryder, and M. A. Ghannoum. 2000. An evaluation of the in vitro activity of terbinafine. Med. Mycol. 38:155-159.

383. Johnson, E. M., A. Szekely, and D. W. Warnock. 1998. In-vitro activity of voriconazole, itraconazole and amphotericin B against filamentous fungi. J. Antimicrob. Chemother. 42:741-745.

384. Johnson, E. M., A. Szekely, and D. W. Warnock. 1999. In vitro activity of Syn-2869, a novel triazole agent, against emerging and less common mold pathogens. Antimicrob. Agents Chemother. 43:1260-1263.

385. Johnson, L. B., and C. A. Kauffman. 2003. Voriconazole: a new triazole antifungal agent. Clin. Infect. Dis. 36:630-637.

386. Juma, A. 1993. Phialophora richardsiae endocarditis of aortic and mitral valves in a diabetic man with a porcine mitral valve. J. Infect. 27:173-175.

387. Jumaa, P. A., C. Lightowler, L. R. Baker, and S. S. Das. 1995. Cutaneous infection caused by Phialophora richardsiae treated successfully by surgical excision in an immunocompromised patient. J. Infect. 30:261-262.

388. Jurkunas, U., I. Behlau, and K. Colby. 2009. Fungal keratitis: changing pathogens and risk factors. Cornea 28:638-643.

389. Kaczmarski, E. B., J. A. Liu Yin, J. A. Tooth, E. M. Love, and I. W. Delamore. 1986. Systemic infection with Aureobasidium pullulans in a leukaemic patient. J. Infect. 13:289-291.

390. Kaell, A. T., and I. Weitzman. 1983. Acute monoarticular arthritis due to Phialophora parasitica. Am. J. Med. 74:519-522.

391. Kahn, J. N., M. J. Hsu, F. Racine, R. Giacobbe, and M. Motyl. 2006 Caspofungin susceptibility in Aspergillus and non-Aspergillus molds: inhibition of glucan synthase and reduction of beta-D-1,3 glucan levels in culture. Antimicrob. Agents Chemother. 50:2214-2216.

392. Kainer, M. A., H. Keshavarz, B. J. Jensen, M. J. Arduino, M. E. Brandt, A. A. Padhye, W. R. Jarvis, and L. K. Archibald. 2005. Saline-filled breast implant contamination with Curvularia species among women who underwent cosmetic breast augmentation. J. Infect. Dis. 192:170-177.

393. Kaltseis, J., J. Rainer, and G. S. De Hoog. 2009. Ecology of Pseudallescheria and Scedosporium species in human-dominated and natural environments and their distribution in clinical samples. Med. Mycol. 47:398-405.

394. Kanj, S. S., S. S. Amr, and G. D. Roberts. 2001. Ramichloridium mackenziei brain abscess: report of two cases and review of the literature. Med. Mycol. 39:97-102.

395. Kantarcioglu, A. S., and G. S. de Hoog. 2004. Infections of the central nervous system by melanized fungi: a review of cases presented between 1999 and 2004. Mycoses 47:4-13.

396. Kantarcioglu, A. S., A. Yucel, and G. S. De Hoog. 2002. Case report. Isolation of Cladosporium cladosporioides from cerebrospinal fluid. Mycoses 45:500-503.

397. Karim, M., H. Sheikh, M. Alam, and Y. Sheikh. 1993. Disseminated Bipolaris infection in an asthmatic patient: case report. Clin. Infect. Dis. 17:248253.

398. Karuppal, R., C. M. Kumaran, A. Marthya, C. V. Manoj Kumar, M. P. Narayanan, R. V. Raman, and S. Thomas. 2009. Tibial osteomyelitis due to Fonsecaea pedrosoi in an immunocompetent patient: case report. J. Foot Ankle Surg. 48:569-572.

399. Kashgari, T. Q., H. Al-Miniawi, and M. K. Moawad Hanna. 2000. Cerebral phaeohyphomycosis caused by Ramichloridium mackenziei in the Eastern Province of Saudi Arabia. Ann. Saudi Med. 20:457-460.

400. Kaufman, S. M. 1971. Curvularia endocarditis following cardiac surgery. Am. J. Clin. Pathol. 56:466-470.

401. Keating, G. M. 2005. Posaconazole. Drugs 65:1553-1567.

402. Kenney, R. T., K. J. Kwon-Chung, A. T. Waytes, D. A. Melnick, H. I. Pass, M. J. Merino, and J. I. Gallin. 1992. Successful treatment of systemic Exophiala dermatitidis infection in a patient with chronic granulomatous disease. Clin. Infect. Dis. 14:235-242.

403. Kent, D., T. Wong, R. Osgood, K. Kosinski, G. Coste, and D. Bor. 1998 Fungemia due to Hormonema dematioides following intense avian exposure. Clin. Infect. Dis. 26:759-760.

404. Kerkmann, M. L., K. Piontek, H. Mitze, and G. Haase. 1999. Isolation of
Exophiala (Wangiella) dermatitidis in a case of otitis externa. Clin. Infect. Dis. 29:939-940.

405. Kerr, C. M., J. R. Perfect, P. C. Craven, J. H. Jorgensen, D. J. Drutz, J. D. Shelburne, H. A. Gallis, and R. A. Gutman. 1983. Fungal peritonitis in patients on continuous ambulatory peritoneal dialysis. Ann. Intern. Med. 99:334-336.

406. Kesson, A. M., M. C. Bellemore, T. J. O’Mara, D. H. Ellis, and T. C. Sorrell. 2009. Scedosporium prolificans osteomyelitis in an immunocompetent child treated with a novel agent, hexadecylphospocholine (miltefosine), in combination with terbinafine and voriconazole: a case report. Clin. Infect. Dis. 48:1257-1261.

407. Kethireddy, S., and D. Andes. 2007. CNS pharmacokinetics of antifungal agents. Expert Opin. Drug Metab. Toxicol. 3:573-581.

408. Keyser, A., F. X. Schmid, H. J. Linde, J. Merk, and D. E. Birnbaum. 2002. Disseminated Cladophialophora bantiana infection in a heart transplant recipient. J. Heart Lung Transplant. 21:503-505.

409. Khan, J. A., S. T. Hussain, S. Hasan, P. McEvoy, and A. Sarwari. 2000. Disseminated Bipolaris infection in an immunocompetent host: an atypical presentation. J. Pak. Med. Assoc. 50:68-71.

410. Khan, Z. U., S. J. Lamdhade, M. Johny, J. Al Khalidi, A. Thussu, H. N. Yossef, I. Al Obaid, and A. A. Nasser. 2002. Additional case of Ramichloridium mackenziei cerebral phaeohyphomycosis from the Middle East. Med. Mycol. 40:429-433.

411. Kiehn, T. E., B. Polsky, E. Punithalingam, F. F. Edwards, A. E. Brown, and D. Armstrong. 1987. Liver infection caused by Coniothyrium fuckelii in a patient with acute myelogenous leukemia. J. Clin. Microbiol. 25:2410-2412.

412. Kim, R. C., C. J. Hodge, Jr., H. V. Lamberson, Jr., and L. B. Weiner. 1981 Traumatic intracerebral implantation of Cladosporium trichoides. Neurology 31:1145-1148.

413. Kimura, M., A. Goto, T. Furuta, T. Satou, S. Hashimoto, and K. Nishimura. 2003. Multifocal subcutaneous phaeohyphomycosis caused by Phialophora verrucosa. Arch. Pathol. Lab. Med. 127:91-93.

414. Kimura, M., and M. R. McGinnis. 1998. Fontana-Masson-stained tissue from culture-proven mycoses. Arch. Pathol. Lab. Med. 122:1107-1111.

415. King, A. B., and T. S. Collette. 1952. Brain abscess due to Cladosporium trichoides. Bull. Johns Hopkins Hosp. 91:298-305.

416. King, D., L. Pasarell, D. M. Dixon, M. R. McGinnis, and W. G. Merz. 1993. A phaeohyphomycotic cyst and peritonitis caused by Phialemonium species and a reevaluation of its taxonomy. J. Clin. Microbiol. 31:1804-1810.

417. Kobayashi, H., A. Sano, N. Aragane, M. Fukuoka, M. Tanaka, F. Kawaura, Y. Fukuno, E. Matsuishi, and S. Hayashi. 2008. Disseminated infection by Bipolaris spicifera in an immunocompetent subject. Med. Mycol. 46:361365 .

418. Kondo, Y., M. Hiruma, A. Matsushita, S. Matsuba, K. Nishimura, and K. Takamori. 2007. Cutaneous phaeohyphomycosis caused by Veronaea botryosa observed as sclerotic cells in tissue. Int. J. Dermatol. 46:625-627.

419. Koo, S., J. M. Bryar, J. H. Page, L. R. Baden, and F. M. Marty. 2009. Diagnostic performance of the $(1,3)$-beta-D-glucan assay for invasive fungal disease. Clin. Infect. Dis. 49:1650-1659.

420. Koo, S., M. Klompas, F. M. Marty, J. M. Bryar, J. H. Page, and L. R. Baden. 2010. Fonsecaea monophora cerebral phaeohyphomycosis: case report of successful surgical excision and voriconazole treatment and review. Med. Mycol. 48:769-774.

421. Kotylo, P. K., K. S. Israel, J. S. Cohen, and M. S. Bartlett. 1989. Subcutaneous phaeohyphomycosis of the finger caused by Exophiala spinifera. Am. J. Clin. Pathol. 91:624-627.

422. Kralovic, S. M., and J. C. Rhodes. 1995. Phaeohyphomycosis caused by Dactylaria (human dactylariosis): report of a case with review of the literature. J. Infect. 31:107-113.

423. Krcmery, V., Jr., S. Spanik, A. Danisovicova, Z. Jesenska, and M. Blahova. 1994. Aureobasidium mansoni meningitis in a leukemia patient successfully treated with amphotericin B. Chemotherapy 40:70-71.

424. Krisher, K. K., N. B. Holdridge, M. M. Mustafa, M. G. Rinaldi, and D. A. McGough. 1995. Disseminated Microascus cirrosus infection in pediatric bone marrow transplant recipient. J. Clin. Microbiol. 33:735-737.

425. Kuhn, F. A., and A. R. Javer. 2000. Allergic fungal rhinosinusitis: perioperative management, prevention of recurrence, and role of steroids and antifungal agents. Otolaryngol. Clin. N. Am. 33:419-433.

426. Kumarasinghe, S. P., and M. P. Kumarasinghe. 2000. Itraconazole pulse therapy in chromoblastomycosis. Eur. J. Dermatol. 10:220-222.

427. Kusenbach, G., H. Skopnik, G. Haase, F. Friedrichs, and H. Dohmen. 1992. Exophiala dermatitidis pneumonia in cystic fibrosis. Eur. J. Ped. 151:344346.

428. Kwon-Chung, K. J., I. Polacheck, and T. J. Popkin. 1982. Melanin-lacking mutants of Cryptococcus neoformans and their virulence for mice. J. Bacteriol. 150:1414-1421.

429. Kwon-Chung, K. J., I. S. Schwartz, and B. J. Rybak. 1975. A pulmonary fungus ball produced by Cladosporium cladosporioides. Am. J. Clin. Pathol. 64:564-568.

430. Labarca, J. A., E. A. Wagar, A. E. Grasmick, H. M. Kokkinos, and D. A Bruckner. 1998. Critical evaluation of 4-week incubation for fungal cultures: is the fourth week useful? J. Clin. Microbiol. 36:3683-3685. 
431. Lacroix, C., E. de Kerviler, P. Morel, F. Derouin, and M. Feuilhade de Chavin. 2005. Madurella mycetomatis mycetoma treated successfully with oral voriconazole. Br. J. Dermatol. 152:1067-1068.

432. Lake, F. R., J. H. Froudist, R. McAleer, R. L. Gillon, A. E. Tribe, and P. J. Thompson. 1991. Allergic bronchopulmonary fungal disease caused by $\mathrm{Bi}$ polaris and Curvularia. Aust. N. Z. J. Med. 21:871-874.

433. Lakshmi, V., C. Padmasri, P. Umabala, C. Sundaram, and M. Panigrahi. 2008. Cerebral phaeohyphomycosis due to Cladophialophora bantiana. Indian J. Med. Microbiol. 26:392-395.

434. Lamaris, G. A., G. Chamilos, R. E. Lewis, A. Safdar, I. I. Raad, and D. P. Kontoyiannis. 2006. Scedosporium infection in a tertiary care cancer center: a review of 25 cases from 1989-2006. Clin. Infect. Dis. 43:1580-1584.

435. Lampert, R. P., J. H. Hutto, W. H. Donnelly, and S. T. Shulman. 1977 Pulmonary and cerebral mycetoma caused by Curvularia pallescens. J. Pediatr. 91:603-605.

436. Langdon, J. S., and W. L. McDonald. 1987. Cranial Exophiala pisciphile infection in Salmo salar in Australia. Bull. Eur. Assoc. Fish Pathol. 7:35-37.

437. Langfelder, K., M. Streibel, B. Jahn, G. Haase, and A. A. Brakhage. 2003 Biosynthesis of fungal melanins and their importance for human pathogenic fungi. Fungal Genet. Biol. 38:143-158.

438. Langvad, F., O. Pedersen, and K. Engjom. 1985. A fungal disease caused by Exophiala sp. nova in farmed Atlantic salmon in Western Norway, p. 323 328. In A. E. Ellis (ed.), Fish and shellfish pathology. Academic Press, London, United Kingdom.

439. Lanisnik Rizner, T., and M. H. Wheeler. 2003. Melanin biosynthesis in the fungus Curvularia lunata (teleomorph: Cochliobolus lunatus). Can. J. Microbiol. 49:110-119.

440. Larangeira de Almeida, H., Jr., R. N. Dallazem, L. S. Dossantos, and S. A Hallal. 2007. Bilateral tinea nigra in a temperate climate. Dermatol. Online J. 13:25.

441. LaRocco, M. T. 2007. Reagents, stains, and media: mycology, p. 1737-1744 In P. R. Murray, E. J. Baron, J. H. Jorgensen, M. L. Landry, and M. A Pfaller (ed.), Manual of clinical microbiology, 9th ed. ASM Press, Washington, DC

442. Lasala, P. R., M. B. Smith, M. R. McGinnis, K. Sackey, J. A. Patel, and S. Qiu. 2005. Invasive Exserohilum sinusitis in a patient with aplastic anemia. Pediatr. Infect. Dis. J. 24:939-941.

443. Lastoria, C., A. Cascina, F. Bini, A. Di Matteo, C. Cavanna, C. Farina, E. Carretto, and F. Meloni. 2009. Pulmonary Cladophialophora boppii infection in a lung transplant recipient: case report and literature review. J. Heart Lung Transplant. 28:635-637.

444. Latham, R. H. 2000. Bipolaris spicifera meningitis complicating a neurosurgical procedure. Scand. J. Infect. Dis. 32:102-103.

445. Lau, A., S. Chen, T. Sorrell, D. Carter, R. Malik, P. Martin, and C Halliday. 2007. Development and clinical application of a panfungal PCR assay to detect and identify fungal DNA in tissue specimens. J. Clin. Microbiol. 45:380-385.

446. Lavelle, P. 1980. Chromoblastomycosis in Mexico. PAHO Sci. Publ. 396: 235-247.

447. Lee, D. K., and A. K. Schwartz. 2007. Primary mycetoma osteomyelitis of the calcaneus with active subcutaneous nodules. J. Foot Ankle Surg. 46: 302-306.

448. Lee, Y. M., P. A. Tambyah, K. H. Lee, K. C. Tan, and S. G. Lim. 2003 Successful treatment of Xylohypha bantiana brain abscess mimicking invasive cerebral aspergillosis in a liver transplant recipient. J. Infect. 47:348351.

449. Lesire, V., E. Hazouard, P. F. Dequin, M. Delain, M. Therizol-Ferly, and A Legras. 1999. Possible role of Chaetomium globosum in infection afte autologous bone marrow transplantation. Intensive Care Med. 25:124-125.

450. Levin, T. P., D. E. Baty, T. Fekete, A. L. Truant, and B. Suh. 2004 Cladophialophora bantiana brain abscess in a solid-organ transplant recipient: case report and review of the literature. J. Clin. Microbiol. 42:43744378 .

451. Levy, I., J. Stein, S. Ashkenazi, Z. Samra, G. Livni, and I. Yaniv. 2003 Ecthyma gangrenosum caused by disseminated Exserohilum in a child with leukemia: a case report and review of the literature. Pediatr. Dermatol. 20:495-497.

452. Li, D. M., R. Y. Li, G. S. De Hoog, Y. X. Wang, and D. L. Wang. 2009 Exophiala asiatica, a new species from a fatal case in China. Med. Mycol. 47:101-109.

453. Li, J. Y., T. Y. Yong, D. I. Grove, and P. T. Coates. 2008. Successful control of Scedosporium prolificans septic arthritis and probable osteomyelitis without radical surgery in a long-term renal transplant recipient. Transpl. Infect. Dis. 10:63-65.

454. Li, R. K., and M. G. Rinaldi. 1999. In vitro antifungal activity of nikkomycin $\mathrm{Z}$ in combination with fluconazole or itraconazole. Antimicrob. Agents Chemother. 43:1401-1405.

455. Lichon, V., and A. Khachemoune. 2006. Mycetoma: a review. Am. J. Clin. Dermatol. 7:315-321

456. Lin, R. Y., and K. D. Williams. 2003. Hypersensitivity to molds in New York City in adults who have asthma. Allergy Asthma Proc. 24:13-18.

457. Lin, S. C., P. L. Sun, Y. M. Ju, and Y. J. Chan. 2009. Cutaneous phaeohy- phomycosis caused by Exserohilum rostratum in a patient with cutaneous T-cell lymphoma. Int. J. Dermatol. 48:295-298.

458. Lirng, J. F., R. D. Tien, A. K. Osumi, J. F. Madden, R. P. McLendon, and D. Sexton. 1995. Cerebral phaeohyphomycosis complicated with brain abscess: a case report. Chin. Med. J. 55:491-495.

459. Little, M. G., and M. L. Hammond. 1995. Scytalidium dimidiatum in Australia. Australas. J. Dermatol. 36:204-205.

460. Liu, G. Y., and V. Nizet. 2009. Color me bad: microbial pigments as virulence factors. Trends Microbiol. 17:406-413.

461. Lopes, J. O., S. H. Alves, J. P. Benevenga, F. B. Brauner, M. S. Castro, and E. Melchiors. 1994. Curvularia lunata peritonitis complicating peritoneal dialysis. Mycopathologia 127:65-67.

462. Loulergue, P., A. Hot, E. Dannaoui, A. Dallot, S. Poiree, B. Dupont, and O. Lortholary. 2006. Successful treatment of black-grain mycetoma with voriconazole. Am. J. Trop. Med. Hyg. 75:1106-1107.

463. Lumbsch, H. T., and S. M. Huhndorf. 2007. Outline of Ascomycota. Myconet 13:1-58

464. Lundstrom, T. S., M. R. Fairfax, M. C. Dugan, J. A. Vazquez, P. H. Chandrasekar, E. Abella, and C. Kasten-Sportes. 1997. Phialophora verrucosa infection in a BMT patient. Bone Marrow Transplant. 20:789-791.

465. Luque, P., F. A. Garcia-Gil, J. Larraga, B. Jimenez, E. Tome-Zelaya, M. T. Serrano, and M. E. Barrao. 2006. Treatment of cutaneous infection by Alternaria alternata with voriconazole in a liver transplant patient. Transplant. Proc. 38:2514-2515.

466. Lye, W. C. 1993. Peritonitis due to Wangiella dermatitidis in a patient on CAPD. Perit. Dial. Int. 13:319-320.

467. Lyons, M. K., J. E. Blair, and K. O. Leslie. 2005. Successful treatment with voriconazole of fungal cerebral abscess due to Cladophialophora bantiana. Clin. Neurol. Neurosurg. 107:532-534.

468. Machard, B., P. Misslin, and M. Lemaire. 1989. Influence of plasma protein binding on the brain uptake of an antifungal agent, terbinafine, in rats. J. Pharm. Pharmacol. 41:700-704.

469. Madan, V., D. Bisset, P. Harris, S. Howard, and M. H. Beck. 2006. Phaeohyphomycosis caused by Exophiala salmonis. Br. J. Dermatol. 155:1082 1084.

470. Madrigal, V., J. Alonso, E. Bureo, F. J. Figols, and R. Salesa. 1995. Fatal meningoencephalitis caused by Scedosporium inflatum (Scedosporium prolificans) in a child with lymphoblastic leukemia. Eur. J. Clin. Microbiol. Infect. Dis. 14:601-603.

471. Maertens, J., K. Lagrou, H. Deweerdt, I. Surmont, G. E. Verhoef, J. Verhaegen, and M. A. Boogaerts. 2000. Disseminated infection by Scedospo rium prolificans: an emerging fatality among haematology patients. Case report and review. Ann. Hematol. 79:340-344.

472. Magnon, K. C., M. Jalbert, and A. A. Padhye. 1993. Osteolytic phaeohyphomycosis caused by Phialemonium obovatum. Arch. Pathol. Lab. Med. 117:841-843.

473. Maiti, P. K., A. Ray, and S. Bandyopadhyay. 2002. Epidemiological aspects of mycetoma from a retrospective study of 264 cases in West Bengal. Trop. Med. Int. Health 7:788-792.

474. Malekzadeh, M., G. D. Overturf, S. B. Auerbach, L. Wong, and M. Hirsch. 1990. Chronic, recurrent osteomyelitis caused by Scedosporium inflatum. Pediatr. Infect. Dis. J. 9:357-359.

475. Malloch, D., and I. F. Salkin. 1984. A new species of Scedosporium associated with osteomyelitis in humans. Mycotaxon 21:247-255.

476. Mani, R. S., Y. T. Chickabasaviah, S. Nagarathna, A. Chandramuki, M. R. Shivprakash, J. Vijayan, D. K. Prashantha, P. S. Vasudevan, A. Natarajan, and J. Kovoor. 2008. Cerebral phaeohyphomycosis caused by Scytalidium dimidiatum: a case report from India. Med. Mycol. 46:705-711.

477. Manian, F. A., and M. J. Brischetto. 1993. Pulmonary infection due to Exophiala jeanselmei: successful treatment with ketoconazole. Clin. Infect. Dis. 16:445-446.

478. Manzouri, B., G. C. Vafidis, and R. K. Wyse. 2001. Pharmacotherapy of fungal eye infections. Expert Opin. Pharmacother. 2:1849-1857.

479. Marimon, R., J. Cano, J. Gene, D. A. Sutton, M. Kawasaki, and J. Guarro. 2007. Sporothrix brasiliensis, S. globosa, and S. mexicana, three new Sporothrix species of clinical interest. J. Clin. Microbiol. 45:3198-3206.

480. Marimon, R., J. Gene, J. Cano, L. Trilles, M. Dos Santos Lazera, and J. Guarro. 2006. Molecular phylogeny of Sporothrix schenckii. J. Clin. Microbiol. 44:3251-3256.

481. Marine, M., F. J. Pastor, and J. Guarro. 2009. Combined antifungal therapy in a murine model of disseminated infection by Cladophialophora bantiana. Med. Mycol. 47:45-49.

482. Markham, W. D., R. D. Key, A. A. Padhye, and L. Ajello. 1990. Phaeohyphomycotic cyst caused by Tetraploa aristata. J. Med. Vet. Mycol. 28:147150.

483. Marques, A. R., K. J. Kwon-Chung, S. M. Holland, M. L. Turner, and J. I. Gallin. 1995. Suppurative cutaneous granulomata caused by Microascus cinereus in a patient with chronic granulomatous disease. Clin. Infect. Dis 20:110-114.

484. Marques, S. A., R. M. Camargo, R. C. Summerbell, G. S. De Hoog, P. Ishioka, L. M. Chambo-Cordaro, and M. E. Marques. 2006. Subcutaneous 
phaeohyphomycosis caused by Phaeoacremonium parasiticum in a renal transplant patient. Med. Mycol. 44:671-676.

485. Marriott, D. J., K. H. Wong, E. Aznar, J. L. Harkness, D. A. Cooper, and D. Muir. 1997. Scytalidium dimidiatum and Lecythophora hoffmannii: unusual causes of fungal infections in a patient with AIDS. J. Clin. Microbiol. 35:2949-2952.

486. Martinez-Gonzalez, M. C., M. M. Verea, D. Velasco, F. Sacristan, J. Del Pozo, J. Garcia-Silva, and E. Fonseca. 2008. Three cases of cutaneous phaeohyphomycosis by Exophiala jeanselmei. Eur. J. Dermatol. 18:313-316.

487. Maslen, M. M., T. Collis, and R. Stuart. 1996. Lasiodiplodia theobromae isolated from a subcutaneous abscess in a Cambodian immigrant to Australia. J. Med. Vet. Mycol. 34:279-283.

488. Mathews, M. S., and S. V. Maharajan. 1999. Exserohilum rostratum causing keratitis in India. Med. Mycol. 37:131-132.

489. Matos, T., G. S. de Hoog, A. G. de Boer, I. de Crom, and G. Haase. 2002 High prevalence of the neurotrope Exophiala dermatitidis and related oligotrophic black yeasts in sauna facilities. Mycoses 45:373-377.

490. Matos, T., G. Haase, A. H. Gerrits van den Ende, and G. S. de Hoog. 2003 Molecular diversity of oligotrophic and neurotropic members of the black yeast genus Exophiala, with accent on E. dermatitidis. Antonie Van Leeuwenhoek 83:293-303.

491. Matsui, T., K. Nishimoto, S. Udagawa, H. Ishihara, and T. Ono. 1999 Subcutaneous phaeohyphomycosis caused by Phaeoacremonium rubrigenum in an immunosuppressed patient. Nippon Ishinkin. Gakkai Zasshi. 40:99102.

492. Matsumoto, T., A. A. Padhye, L. Ajello, and P. G. Standard. 1984. Critical review of human isolates of Wangiella dermatitidis. Mycologia 76:232-249.

493. Matsumoto, T., L. Ajello, T. Matsuda, P. J. Szaniszlo, and T. J. Walsh 1994. Developments in hyalohyphomycosis and phaeohyphomycosis. J. Med. Vet. Mycol. 32(Suppl. 1):329-349.

494. Matsumoto, T., K. Nishimoto, K. Kimura, A. A. Padhye, L. Ajello, and M. R. McGinnis. 1984. Phaeohyphomycosis caused by Exophiala moniliae. Sabouraudia 22:17-26.

495. Matsushita, A., L. Jilong, M. Hiruma, M. Kobayashi, T. Matsumoto, H. Ogawa, and A. A. Padhye. 2003. Subcutaneous phaeohyphomycosis caused by Veronaea botryosa in the People's Republic of China. J. Clin. Microbiol. 41:2219-2222.

496. Matsuwaki, Y., K. Wada, T. A. White, L. M. Benson, M. C. Charlesworth, J. L. Checkel, Y. Inoue, K. Hotta, J. U. Ponikau, C. B. Lawrence, and H. Kita. 2009. Recognition of fungal protease activities induces cellular activation and eosinophil-derived neurotoxin release in human eosinophils. J. Immunol. 183:6708-6716.

497. Mayser, P., M. Nilles, and G. S. De Hoog. 2002. Case report. Cutaneous phaeohyphomycosis due to Alternaria alternata. Mycoses 45:338-340.

498. McAleer, R., D. B. Kroenert, J. L. Elder, and J. H. Froudist. 1981. Allergic bronchopulmonary disease caused by Curvularia lunata and Drechslera hawaiiensis. Thorax 36:338-344.

499. McCown, H. F., and E. E. Sahn. 1997. Subcutaneous phaeohyphomycosis and nocardiosis in a kidney transplant patient. J. Am. Acad. Dermatol. 36:863-866.

500. McGill, H. C., and J. W. Brueck. 1956. Brain abscess due to Hormodendrum species. Arch. Pathol. 62:303-311.

501. McGinnis, M. R. 1983. Chromoblastomycosis and phaeohyphomycosis: new concepts, diagnosis, and mycology. J. Am. Acad. Dermatol. 8:1-16.

502. McGinnis, M. R. 1996. Mycetoma. Dermatol. Clin. 14:97-104.

503. McGinnis, M. R., A. A. Padhye, and L. Ajello. 1982. Pseudallescheria boydit Negroni et Fischer, 1943, and its later synonym Petriellidium Mallock, 1970. Mycotaxon 14:94-102.

504. McGinnis, M. R., and A. A. Padhye. 2003. Fungi causing eumycotic mycetoma, p. 1848-1856. In P. R. Murray, E. J. Baron, J. H. Jorgensen, M. A Pfaller, and R. H. Yolken (ed.), Manual of clinical microbiology, 8th ed. ASM Press, Washington, DC.

505. McGinnis, M. R., S. M. Lemon, D. H. Walker, G. S. De Hoog, and G. Haase 1999. Fatal cerebritis caused by a new species of Cladophialophora. Stud. Mycol. 43:166-171.

506. McGinnis, M. R., and L. Pasarell. 1998. In vitro evaluation of terbinafine and itraconazole against dematiaceous fungi. Med. Mycol. 36:243-246.

507. McGinnis, M. R., and L. Pasarell. 1998. In vitro testing of susceptibilities of filamentous ascomycetes to voriconazole, itraconazole, and amphotericin B, with consideration of phylogenetic implications. J. Clin. Microbiol. 36: 2353-2355.

508. McGinnis, M. R., M. G. Rinaldi, and R. E. Winn. 1986. Emerging agents of phaeohyphomycosis: pathogenic species of Bipolaris and Exserohilum. J. Clin. Microbiol. 24:250-259.

509. Meletiadis, J., J. F. Meis, J. W. Mouton, J. L. Rodriquez-Tudela, J. P. Donnelly, and P. E. Verweij. 2002. In vitro activities of new and conventional antifungal agents against clinical Scedosporium isolates. Antimicrob. Agents Chemother. 46:62-68.

510. Meletiadis, J., J. W. Mouton, J. F. Meis, and P. E. Verweij. 2003. In vitro drug interaction modeling of combinations of azoles with terbinafine against clinical Scedosporium prolificans isolates. Antimicrob. Agents Chemother. 47:106-117.
511. Meletiadis, J., J. W. Mouton, J. L. Rodriguez-Tudela, J. F. Meis, and P. E. Verweij. 2000. In vitro interaction of terbinafine with itraconazole against clinical isolates of Scedosporium prolificans. Antimicrob. Agents Chemother. 44:470-472.

512. Menon, S., and J. C. Edwards. 1994. Mycotic arthritis of the knee due to Madurella grisea. Br. J. Rheumatol. 33:292-295.

513. Middleton, F. G., P. F. Jurgenson, J. P. Utz, S. Shadomy, and H. J. Shadomy. 1976. Brain abscess caused by Cladosporium trichoides. Arch. Intern. Med. 136:444-448.

514. Miele, P. S., C. S. Levy, M. A. Smith, E. M. Dugan, R. H. Cooke, J. A. Light, and D. R. Lucey. 2002. Primary cutaneous fungal infections in solid organ transplantation: a case series. Am. J. Transplant. 2:678-683.

515. Minotto, R., C. D. Bernardi, L. F. Mallmann, M. I. Edelweiss, and M. L. Scroferneker. 2001. Chromoblastomycosis: a review of 100 cases in the state of Rio Grande do Sul, Brazil. J. Am. Acad. Dermatol. 44:585-592.

516. Mitchell, D. M., M. Fitz-Henley, and J. Horner-Bryce. 1990. A case of disseminated phaeohyphomycosis caused by Cladosporium devriesii. West Indian Med. J. 39:118-123

517. Mohammedi, I., M. A. Piens, C. Audigier-Valette, J. C. Gantier, L. Argaud, O. Martin, and D. Robert. 2004. Fatal Microascus trigonosporus (anamorph Scopulariopsis) pneumonia in a bone marrow transplant recipient. Eur. J. Clin. Microbiol. Infect. Dis. 23:215-217.

518. Moore, M. K. 1986. Hendersonula toruloidea and Scytalidium hyalinum infections in London, England. J. Med. Vet. Mycol. 24:219-230.

519. Moore, M. K. 1992. The infection of human skin and nail by Scytalidium species. Curr. Top. Med. Mycol. 4:1-42.

520. Morris, A., W. A. Schell, D. McDonagh, S. Chaffee, and J. R. Perfect. 1995 Pneumonia due to Fonsecaea pedrosoi and cerebral abscesses due to Emericella nidulans in a bone marrow transplant recipient. Clin. Infect. Dis. 21:1346-1348.

521. Morris-Jones, R., B. L. Gomez, S. Diez, M. Uran, S. D. Morris-Jones, A. Casadevall, J. D. Nosanchuk, and A. J. Hamilton. 2005. Synthesis of melanin pigment by Candida albicans in vitro and during infection. Infect. Immun. 73:6147-6150.

522. Morris-Jones, R., S. Youngchim, J. M. Hextall, B. L. Gomez, S. D. MorrisJones, R. J. Hay, A. Casadevall, J. D. Nosanchuk, and A. J. Hamilton. 2004. Scytalidium dimidiatum causing recalcitrant subcutaneous lesions produces melanin. J. Clin. Microbiol. 42:3789-3794.

523. Morton, S. J., K. Midthun, and W. G. Merz. 1986. Granulomatous encephalitis caused by Bipolaris hawaiiensis. Arch. Pathol. Lab. Med. 110:11831185 .

524. Moskowitz, L. B., T. J. Cleary, M. R. McGinnis, and C. B. Thomson. 1983. Phialophora richardsiae in a lesion appearing as a giant cell tumor of the tendon sheath. Arch. Pathol. Lab. Med. 107:374-376.

525. Mostert, L., J. Z. Groenewald, R. C. Summerbell, V. Robert, D. A. Sutton, A. A. Padhye, and P. W. Crous. 2005. Species of Phaeoacremonium associated with infections in humans and environmental reservoirs in infected woody plants. J. Clin. Microbiol. 43:1752-1767.

526. Mroueh, S., and A. Spock. 1992. Allergic bronchopulmonary disease caused by Curvularia in a child. Ped. Pulm. 12:123-126.

527. Mukherji, S. K., and M. Castillo. 1995. Cerebral phaeohyphomycosis caused by Xylohypha bantiana: MR findings. Am. J. Roentgenol. 164:13041305 .

528. Mullane, K., A. A. Toor, C. Kalnicky, T. Rodriguez, J. Klein, and P. Stiff. 2007. Posaconazole salvage therapy allows successful allogeneic hematopoietic stem cell transplantation in patients with refractory invasive mold infections. Transpl. Infect. Dis. 9:89-96.

529. Musella, R. A., and G. H. Collins. 1971. Cerebral chromoblastomycosis Case report. J. Neurosurg. 35:219-222.

530. Nadkarni, T. D., A. Goel, A. Shenoy, and A. P. Karapurkar. 1993. Cladosporium bantianum (trichoides) infection of the brain. J. Postgrad. Med. 39:43-44.

531. Naim, U. R., E. S. Mahgoub, and A. H. Chagla. 1988. Fatal brain abscesses caused by Ramichloridium obovoideum: report of three cases. Acta Neurochirurg. 93:92-95.

532. Najafzadeh, M. J., H. Badali, M. T. Illnait-Zaragozi, G. S. De Hoog, and J. F. Meis. 2010. In vitro activities of eight antifungal drugs against 55 clinical isolates of Fonsecaea spp. Antimicrob. Agents Chemother. 54:16361638.

533. Najafzadeh, M. J., C. Gueidan, H. Badali, A. H. Van Den Ende, L. Xi, and G. S. De Hoog. 2009. Genetic diversity and species delimitation in the opportunistic genus Fonsecaea. Med. Mycol. 47:17-25.

534. National Committe for Clinical Laboratory Standards. 1997. Reference method for broth dilution antifungal susceptibility testing of yeasts. Approved standard M27-A. National Committe for Clinical Laboratory Standards, Wayne, PA.

535. Ndiaye, B., M. Develoux, M. T. Dieng, A. Kane, O. Ndir, G. Raphenon, and M. Huerre. 2000. Current report of mycetoma in Senegal: report of 109 cases. J. Mycol. Méd. 10:140-144.

536. Negroni, R., S. H. Helou, N. Petri, A. M. Robles, A. Arechavala, and M. H. Bianchi. 2004. Case study: posaconazole treatment of disseminated phaeohyphomycosis due to Exophiala spinifera. Clin. Infect. Dis. 38:e15-20. 
537. Negroni, R., A. Tobon, B. Bustamante, M. A. Shikanai-Yasuda, H. Patino, and A. Restrepo. 2005. Posaconazole treatment of refractory eumycetoma and chromoblastomycosis. Rev. Inst. Med. Trop. Sao Paulo 47:339-346.

538. Nenoff, P., U. Gutz, K. Tintelnot, A. Bosse-Henck, M. Mierzwa, J. Hofmann, L. C. Horn, and U. F. Haustein. 1996. Disseminated mycosis due to Scedosporium prolificans in an AIDS patient with Burkitt lymphoma. Mycoses 39:461-465.

539. Neoh, C. Y., S. H. Tan, and P. Perera. 2007. Cutaneous phaeohyphomycosis due to Cladophialophora bantiana in an immunocompetent patient. Clin. Exp. Dermatol. 32:539-540.

540. Nesky, M. A., E. C. McDougal, and J. E. Peacock, Jr. 2000. Pseudallescheria boydii brain abscess successfully treated with voriconazole and surgical drainage: case report and literature review of central nervous system pseudallescheriasis. Clin. Infect. Dis. 31:673-677.

541. Neukirch, C., C. Henry, B. Leynaert, R. Liard, J. Bousquet, and F. Neukirch. 1999. Is sensitization to Alternaria alternata a risk factor for severe asthma? A population-based study. J. Allergy Clin. Immunol. 103:709-711.

542. Neumeister, B., W. Hartmann, M. Oethinger, B. Heymer, and R. Marre 1994. A fatal infection with Alternaria alternata and Aspergillus terreus in a child with agranulocytosis of unknown origin. Mycoses 37:181-185.

543. Nielsen, K., H. Lang, A. C. Shum, K. Woodruff, and J. D. Cherry. 1993 Disseminated Scedosporium prolificans infection in an immunocompromised adolescent. Pediatr. Infect. Dis. J. 12:882-884.

544. Noble, J. A., S. A. Crow, D. G. Ahearn, and F. A. Kuhn. 1997. Allergic fungal sinusitis in the southeastern U. S. A.: involvement of a new agent Epicoc cum nigrum Ehrenb. ex Schlecht. 1824. J. Med. Vet. Mycol. 35:405-409.

545. Nobrega, J. P., S. Rosemberg, A. M. Adami, E. M. Heins-Vaccari, S. Lacaz Cda, and T. de Brito. 2003. Fonsecaea pedrosoi cerebral phaeohyphomycosis ("chromoblastomycosis"): first human culture-proven case reported in Brazil. Rev. Inst. Med. Trop. Sao Paulo. 45:217-220.

546. Nosanchuk, J. D., and A. Casadevall. 2003. The contribution of melanin to microbial pathogenesis. Cell. Microbiol. 5:203-223.

547. Nosanchuk, J. D., and A. Casadevall. 2006. Impact of melanin on microbial virulence and clinical resistance to antimicrobial compounds. Antimicrob. Agents Chemother. 50:3519-3528.

548. Nosanchuk, J. D., B. L. Gomez, S. Youngchim, S. Diez, P. Aisen, R. M Zancope-Oliveira, A. Restrepo, A. Casadevall, and A. J. Hamilton. 2002 Histoplasma capsulatum synthesizes melanin-like pigments in vitro and during mammalian infection. Infect. Immun. 70:5124-5131.

549. Nosanchuk, J. D., A. L. Rosas, S. C. Lee, and A. Casadevall. 2000. Melanisation of Cryptococcus neoformans in human brain tissue. Lancet $\mathbf{3 5 5}$ 2049-2050.

550. Nucci, M., T. Akiti, G. Barreiros, F. Silveira, S. G. Revankar, D. A. Sutton, and T. F. Patterson. 2001. Nosocomial fungemia due to Exophiala jeanselmei var. jeanselmei and a Rhinocladiella species: newly described causes of bloodstream infection. J. Clin. Microbiol. 39:514-518.

551. Nulens, E., E. De Laere, H. Vandevelde, L. B. Hilbrands, A. J. Rijs, W. J. Melchers, and P. E. Verweij. 2006. Alternaria infectoria phaeohyphomycosis in a renal transplant patient. Med. Mycol. 44:379-382.

552. Oberto-Perdigon, L., H. Romero, M. Perez-Blanco, and R. Apitz-Castro. 2005. An ELISA test for the study of the therapeutic evolution of chromoblastomycosis by Cladophialophora carrionii in the endemic area of Falcon State, Venezuela. Rev. Iberoam. Micol. 22:39-43.

553. Odabasi, Z., V. L. Paetznick, J. R. Rodriguez, E. Chen, and L. OstroskyZeichner. 2004. In vitro activity of anidulafungin against selected clinically important mold isolates. Antimicrob. Agents Chemother. 48:1912-1915.

554. Odell, J. A., S. Alvarez, D. G. Cvitkovich, D. A. Cortese, and B. L. McComb. 2000. Multiple lung abscesses due to Ochroconis gallopavum, a dematiaceous fungus, in a nonimmunocompromised wood pulp worker. Chest 118 : 1503-1505.

555. O'Driscoll, B. R., L. C. Hopkinson, and D. W. Denning. 2005. Mold sensitization is common amongst patients with severe asthma requiring multiple hospital admissions. BMC Pulm. Med. 5:4.

556. Ogawa, M. M. N. Z. Galante, P. Godoy, O. Fischman-Gompertz, F. Martelli, A. L. Colombo, J. Tomimori, and J. O. Medina-Pestana. 2009. Treatment of subcutaneous phaeohyphomycosis and prospective follow-up of 17 kidney transplant recipients. J. Am. Acad. Dermatol. 61:977-985.

557. Ogden, P. E., D. L. Hurley, and P. T. Cain. 1992. Fatal fungal endarteritis caused by Bipolaris spicifera following replacement of the aortic valve. Clin. Infect. Dis. 14:596-598.

558. Ohira, S., K. Isoda, H. Hamanaka, K. Takahashi, K. Nishimoto, and H. Mizutani. 2002. Case report. Phaeohyphomycosis caused by Phialophora verrucosa developed in a patient with non-HIV acquired immunodeficiency syndrome. Mycoses 45:50-54.

559. O'Quinn, R. P., J. L. Hoffmann, and A. S. Boyd. 2001. Colletotrichum species as emerging opportunistic fungal pathogens: a report of 3 cases of phaeohyphomycosis and review. J. Am. Acad. Dermatol. 45:56-61.

560. Ortoneda, M., J. Capilla, I. Pujol, F. J. Pastor, E. Mayayo, J. FernandezBallart, and J. Guarro. 2002. Liposomal amphotericin B and granulocyte colony-stimulating factor therapy in a murine model of invasive infection by Scedosporium prolificans. J. Antimicrob. Chemother. 49:525-529.

561. Osherov, A., E. Schwammenthal, R. Kuperstein, J. Strahilevitz, and M. S.
Feinberg. 2006. Phialemonium curvatum prosthetic valve endocarditis with an unusual echocardiographic presentation. Echocardiography 23:503-505.

562. Osiyemi, O. O., L. M. Dowdy, S. M. Mallon, and T. Cleary. 2001. Cerebra phaeohyphomycosis due to a novel species: report of a case and review of the literature. Transplantation 71:1343-1346.

563. O'Sullivan, F. X., B. R. Stuewe, J. M. Lynch, J. W. Brandsberg, T. B. Wiegmann, R. V. Patak, W. G. Barnes, and G. R. Hodges. 1981. Peritoniti due to Drechslera spicifera complicating continuous ambulatory peritoneal dialysis. Ann. Intern. Med. 94:213-214.

564. Ozbek, Z., S. Kang, J. Sivalingam, C. J. Rapuano, E. J. Cohen, and K. M. Hammersmith. 2006. Voriconazole in the management of Alternaria keratitis. Cornea 25:242-244.

565. Oztas, E., B. Odemis, M. Kekilli, M. Kurt, B. M. Dinc, E. Parlak, A. Kalkanci, and N. Sasmaz. 2009. Systemic phaeohyphomycosis resembling primary sclerosing cholangitis caused by Exophiala dermatitidis. J. Med. Microbiol. 58:1243-1246.

566. Padhye, A. A., L. Ajello, M. A. Wieden, and K. K. Steinbronn. 1986. Phaeohyphomycosis of the nasal sinuses caused by a new species of Exserohilum J. Clin. Microbiol. 24:245-249.

567. Padhye, A. A., M. S. Davis, D. Baer, A. Reddick, K. K. Sinha, and J. Ott. 1998. Phaeohyphomycosis caused by Phaeoacremonium inflatipes. J. Clin. Microbiol. 36:2763-2765.

568. Padhye, A. A., W. B. Helwig, N. G. Warren, L. Ajello, F. W. Chandler, and M. R. McGinnis. 1988. Subcutaneous phaeohyphomycosis caused by Xylohypha emmonsii. J. Clin. Microbiol. 26:709-712.

569. Padhye, A. A., M. R. McGinnis, and L. Ajello. 1978. Thermotolerance of Wangiella dermatitidis. J. Clin. Microbiol. 8:424-426.

570. Palaoglu, S., A. Sav, T. Basak, Y. Yalcinlar, and B. W. Scheithauer. 1993. Cerebral phaeohyphomycosis. Neurosurgery 33:894-897.

571. Palencarova, E., Z. Jesenska, L. Plank, S. Straka, T. Baska, A. Hajtman, and J. Pec. 1995. Phaeohyphomycosis caused by Alternaria species and Phaeosclera dematioides Sigler, Tsuneda and Carmichael. Clin. Exp. Dermatol. 20:419-422.

572. Pang, K. R., J. J. Wu, D. B. Huang, and S. K. Tyring. 2004. Subcutaneous fungal infections. Dermatol. Ther. 17:523-531.

573. Paolo, W. F., Jr., E. Dadachova, P. Mandal, A. Casadevall, P. J. Szaniszlo, and J. D. Nosanchuk. 2006. Effects of disrupting the polyketide synthase gene WdPKS1 in Wangiella [Exophiala] dermatitidis on melanin production and resistance to killing by antifungal compounds, enzymatic degradation, and extremes in temperature. BMC Microbiol. 6:55.

574. Pappagianis, D., and L. Ajello. 1994. Dematiaceous-a mycologic misnomer? J. Med. Vet. Mycol. 32:319-321.

575. Pardo, F., E. Ferrer, P. A. Romero, and M. L. Perez del Molino. 2006 Cerebral phaeiohyphomycosis due to Cladophialophora bantiana. Enferm. Infecc. Microbiol. Clin. 24:593-594.

576. Parra, I. H., R. Galimberti, G. Galimberti, B. Guanella, and A. Kowalczuk. 2008. Lymphocutaneous nocardiosis and cutaneous pheohyphomycosis in a liver transplant recipient. Int. J. Dermatol. 47:571-574.

577. Pastor, F. J., and J. Guarro. 2008. Alternaria infections: laboratory diagnosis and relevant clinical features. Clin. Microbiol. Infect. 14:734-746.

578. Patel, R., C. A. Gustaferro, R. A. Krom, R. H. Wiesner, G. D. Roberts, and C. V. Paya. 1994. Phaeohyphomycosis due to Scopulariopsis brumptii in liver transplant recipient. Clin. Infect. Dis. 19:198-200.

579. Pathengay, A., G. Y. Shah, T. Das, and S. Sharma. 2006. Curvularia lunata endophthalmitis presenting with a posterior capsular plaque. Indian J. Ophthalmol. 54:65-66.

580. Pauzner, R., A. Goldschmied-Reouven, I. Hay, Z. Vered, Z. Ziskind, N. Hassin, and Z. Farfel. 1997. Phaeohyphomycosis following cardiac surgery: case report and review of serious infection due to Bipolaris and Exserohilum species. Clin. Infect. Dis. 25:921-923.

581. Pedroso, A. G. 1920. 4 casos de dermatite verrucosa produzida pela Phialophora verrucosa. Ann. Paulistas Med. Cirurgia 11:53-61.

582. Peerapur, B. V., S. D. Rao, S. Patil, and B. G. Mantur. 2004. Keratomycosis due to Exserohilum rostratum - a case report. Indian J. Med. Microbiol. 22:126-127.

583. Pellon Daben, R., E. Marco de Lucas, L. Martin Cuesta, T. Piedra Velasco, J. Arnaiz Garcia, R. Landeras, M. Lopez Duarte, and A. Bermudez. 2008. Imaging findings of pulmonary infection caused by Scedosporium prolificans in a deep immunocompromised patient. Emerg. Radiol. 15:47-49.

584. Peltroche-Llacsahuanga, H., N. Schnitzler, S. Jentsch, A. Platz, S. De Hoog, K. G. Schweizer, and G. Haase. 2003. Analyses of phagocytosis, evoked oxidative burst, and killing of black yeasts by human neutrophils: a tool for estimating their pathogenicity? Med. Mycol. 41:7-14.

585. Pendle, S., K. Weeks, M. Priest, A. Gill, B. Hudson, G. Kotsiou, and R. Pritchard. 2004. Phaeohyphomycotic soft tissue infections caused by the coelomycetous fungus Microsphaeropsis arundinis. J. Clin. Microbiol. 42: $5315-5319$

586. Pereiro, M., Jr., J. Jo-Chu, and J. Toribio. 1998. Phaeohyphomycotic cyst due to Cladosporium cladosporioides. Dermatology 197:90-92.

587. Pereiro, M., Jr., M. M. Pereiro Ferreiros, G. S. De Hoog, and J. Toribio 2004. Cutaneous infection caused by Alternaria in patients receiving tacrolimus. Med. Mycol. 42:277-282. 
588. Perez, C., M. T. Colella, C. Olaizola, C. Hartung de Capriles, S. Magaldi, and S. Mata-Essayag. 2005. Tinea nigra: report of twelve cases in Venezuela. Mycopathologia 160:235-238.

589. Perez-Blanco, M., G. Fernandez-Zeppenfeldt, R. Hernandez, F. Yegres, and D. Borelli. 1998. Chromomycosis by Rhinocladiella aquaspera: the first case in Venezuela. Rev. Iberoam. Micol. 15:51-54.

590. Petrini, B., F. Farnebo, M. A. Hedblad, and P. Appelgren. 2006. Concomitant late soft tissue infections by Cladophialophora bantiana and Mycobacterium abscessus following tsunami injuries. Med. Mycol. 44:189-192.

591. Pfaller, M. A., and M. R. McGinnis. 2009. The laboratory and clinical mycology, p. 55-77. In E. J. Anaissie, M. R. McGinnis, M. A. Pfaller (ed.), Clinical mycology, 2nd ed. Churchill Livingstone, Philadelphia, PA.

592. Pfaller, M. A., S. A. Messer, R. J. Hollis, and R. N. Jones. 2002. Antifungal activities of posaconazole, ravuconazole, and voriconazole compared to those of itraconazole and amphotericin B against 239 clinical isolates of Aspergillus spp. and other filamentous fungi: report from SENTRY Antimicrobial Surveillance Program, 2000. Antimicrob. Agents Chemother. 46: 1032-1037.

593. Pickles, R. W., D. E. Pacey, D. B. Muir, and W. H. Merrell. 1996. Experience with infection by Scedosporium prolificans including apparent cure with fluconazole therapy. J. Infect. 33:193-197.

594. Piepenbring, M., O. A. Caceres Mendez, A. A. Espino Espinoza, R. Kirschner, and H. Schofer. 2007. Chromoblastomycosis caused by Chaetomium funicola: a case report from Western Panama. Br. J. Dermatol. 157:10251029.

595. Pierach, C. A., G. Gulmen, G. J. Dhar, and J. C. Kiser. 1973. Phialophora mutabilis endocarditis. Ann. Intern. Med. 79:900-901. (Letter.)

596. Pierce, N. F., J. C. Millan, B. S. Bender, and J. L. Curtis. 1986. Disseminated Curvularia infection. Arch. Pathol. Lab. Med. 110:871.

597. Pihet, M., J. Carrere, B. Cimon, D. Chabasse, L. Delhaes, F. Symoens, and J. P. Bouchara. 2009. Occurrence and relevance of filamentous fungi in respiratory secretions of patients with cystic fibrosis-a review. Med. Mycol. 47:387-397.

598. Pimentel, J. D., K. Mahadevan, A. Woodgyer, L. Sigler, C. Gibas, O. C Harris, M. Lupino, and E. Athan. 2005. Peritonitis due to Curvularia inaequalis in an elderly patient undergoing peritoneal dialysis and a review of six cases of peritonitis associated with other Curvularia spp. J. Clin. Microbiol. 43:4288-4292.

599. Pirt, S. J., and B. I. Rowley. 1969. Melanin production in Aspergillus nidulans. Biochem. J. 114:9-10.

600. Pitisuttithum, P., R. Negroni, J. R. Graybill, B. Bustamante, P. Pappas, S Chapman, R. S. Hare, and C. J. Hardalo. 2005. Activity of posaconazole in the treatment of central nervous system fungal infections. J. Antimicrob. Chemother. 56:745-755.

601. Pitrak, D. L. E. W. Koneman, R. C. Estupinan, and J. Jackson, 1988 Phialophora richardsiae infection in humans. Rev. Infect. Dis. 10:1195-1203.

602. Podnos, Y. D., P. Anastasio, L. De La Maza, and R. B. Kim. 1999. Cerebra phaeohyphomycosis caused by Ramichloridium obovoideum (Ramichloridium mackenziei): case report. Neurosurgery 45:372-375.

603. Polak, A. 1984. Antimycotic therapy of experimental infections caused by dematiaceous fungi. Sabouraudia 22:279-289.

604. Porteous, N. B., A. M. Grooters, S. W. Redding, E. H. Thompson, M. G. Rinaldi, G. S. De Hoog, and D. A. Sutton. 2003. Identification of Exophiala mesophila isolated from treated dental unit waterlines. J. Clin. Microbiol. 41:3885-3889.

605. Prasad, K. N., N. Prasad, A. Gupta, R. K. Sharma, A. K. Verma, and A. Ayyagari. 2004. Fungal peritonitis in patients on continuous ambulatory peritoneal dialysis: a single centre Indian experience. J. Infect. 48:96-101.

606. Prenafeta-Boldu, F. X., R. Summerbell, and G. Sybren de Hoog. 2006. Fungi growing on aromatic hydrocarbons: biotechnology's unexpected encounter with biohazard? FEMS Microbiol. Rev. 30:109-130.

607. Pritchard, R. C., and D. B. Muir. 1987. Black fungi: a survey of dematiaceous hyphomycetes from clinical specimens identified over a five-year period in a reference laboratory. Pathology 19:281-284.

608. Proia, L. A., M. K. Hayden, P. L. Kammeyer, J. Ortiz, D. A. Sutton, T. Clark, H. J. Schroers, and R. C. Summerbell. 2004. Phialemonium: an emerging mold pathogen that caused 4 cases of hemodialysis-associated endovascular infection. Clin. Infect. Dis. 39:373-379.

609. Qiu-Xia, C., L. Chang-Xing, H. Wen-Ming, S. Jiang-Qiang, L. Wen, and L. Shun-Fang. 2008. Subcutaneous phaeohyphomycosis caused by Cladosporium sphaerospermum. Mycoses 51:79-80.

610. Queiroz-Telles, F., P. Esterre, M. Perez-Blanco, R. G. Vitale, C. G. Salgado, and A. Bonifaz. 2009. Chromoblastomycosis: an overview of clinical manifestations, diagnosis and treatment. Med. Mycol. 47:3-15.

611. Queiroz-Telles, F., K. S. Purim, J. N. Fillus, G. F. Bordignon, R. P. Lameira, J. Van Cutsem, and G. Cauwenbergh. 1992. Itraconazole in the treatment of chromoblastomycosis due to Fonsecaea pedrosoi. Int. J. Dermatol. 31:805-812.

612. Rabodonirina, M., S. Paulus, F. Thevenet, R. Loire, E. Gueho, O. Bastien, J. F. Mornex, M. Celard, and M. A. Piens. 1994. Disseminated Scedosporium prolificans (S. inflatum) infection after single-lung transplantation. Clin. Infect. Dis. 19:138-142.
613. Rajendran, C., B. K. Khaitan, R. Mittal, M. Ramam, M. Bhardwaj, and K. K. Datta. 2003. Phaeohyphomycosis caused by Exophiala spinifera in India. Med. Mycol. 41:437-441.

614. Rallis, E., and E. Frangoulis. 2006. Successful treatment of subcutaneous phaeohyphomycosis owing to Exophiala jeanselmei with oral terbinafine. Int. J. Dermatol. 45:1369-1370.

615. Rebell, G., and R. K. Forster. 1976. Lasiodiplodia theobromae as a cause of keratomycoses. Sabouraudia 14:155-170.

616. Redondo-Bellon, P., M. Idoate, M. Rubio, and J. Ignacio Herrero. 1997. Chromoblastomycosis produced by Aureobasidium pullulans in an immunosuppressed patient. Arch. Dermatol. 133:663-664.

617. Rees, J. R., R. W. Pinner, R. A. Hajjeh, M. E. Brandt, and A. L. Reingold. 1998. The epidemiological features of invasive mycotic infections in the San Francisco Bay area, 1992-1993: results of population-based laboratory active surveillance. Clin. Infect. Dis. 27:1138-1147.

618. Reiss-Levy, E., and P. Clingan. 1981. Peritonitis caused by Alternaria alternata. Med. J. Aust. 2:44

619. Remon, C., I. J. de la Calle, F. Vallejo Carrion, S. Perez-Ramos, and E. Fernandez Ruiz. 1996. Exophiala jeanselmei peritonitis in a patient on CAPD. Perit. Dial. Int. 16:536-538.

620. Restrepo, A., A. Gonzalez, I. Gomez, M. Arango, and C. de Bedout. 1988. Treatment of chromoblastomycosis with itraconazole. Ann. N. Y. Acad. Sci. 544:504-516.

621. Restrepo, A., M. R. McGinnis, D. Malloch, A. Porras, N. Giraldo, A. Villegas, and J. Herrera. 1984. Fungal endocarditis caused by Arnium leporinum following cardiac surgery. Sabouraudia 22:225-234.

622. Revankar, S. G. 2004. Dematiaceous fungi. Semin. Resp. Crit. Care Med. 25:183-189.

623. Revankar, S. G. 2006. Phaeohyphomycosis. Infect. Dis. Clin. North Am. 20:609-620.

624. Revankar, S. G. 2007. Phaeohyphomycosis, p. 243-252. In C. A. Kauffman (ed.), Atlas of fungal infections, 2nd ed. Current Medicine, Philadelphia, PA.

625. Revankar, S. G. 2005. Therapy of infections caused by dematiaceous fungi. Expert Rev. Anti Infect. Ther. 3:601-612.

626. Revankar, S. G., M. D. Nailor, and J. D. Sobel. 2008. Use of terbinafine in rare and refractory mycoses. Future Microbiol. 3:9-17.

627. Revankar, S. G., J. E. Patterson, D. A. Sutton, R. Pullen, and M. G. Rinaldi. 2002. Disseminated phaeohyphomycosis: review of an emerging mycosis. Clin. Infect. Dis. 34:467-476.

628. Revankar, S. G., D. A. Sutton, and M. G. Rinaldi. 2004. Primary central nervous system phaeohyphomycosis: a review of 101 cases. Clin. Infect. Dis. 38:206-216.

629. Riley, O., and S. H. Mann. 1960. Brain abscess caused by Cladosporioum trichoides. Am. J. Clin. Pathol. 33:525-531.

630. Rinaldi, M. 1996. G. Phaeohyphomycosis. Dermatol. Clin. 14:147-153.

631. Rinaldi, M. G., P. Phillips, J. G. Schwartz, R. E. Winn, G. R. Holt, F. W. Shagets, J. Elrod, G. Nishioka, and T. B. Aufdemorte. 1987. Human Curvularia infections. Report of five cases and review of the literature. Diagn. Microbiol. Infect. Dis. 6:27-39.

632. Rios-Fabra, A., A. R. Moreno, and R. E. Isturiz. 1994. Fungal infection in Latin American countries. Infect. Dis. Clin. North Am. 8:129-154.

633. Rivard, R. G., S. McCall, M. E. Griffith, J. S. Hawley, R. A. Ressner, H. Borra, J. E. Moon, M. L. Beckius, C. K. Murray, and D. R. Hospenthal. 2007. Efficacy of caspofungin and posaconazole in a murine model of disseminated Exophiala infection. Med. Mycol. 45:685-689.

634. Rivero, M., A. Hidalgo, A. Alastruey-Izquierdo, M. Cia, L. Torroba, and J. L. Rodriguez-Tudela. 2009. Infections due to Phialemonium species: case report and review. Med. Mycol. 47:766-774.

635. Robson, A. M., and R. D. Craver. 1994. Curvularia urinary tract infection: a case report. Pediatr. Nephrol. 8:83-84.

636. Roche, M., R. M. Redmond, S. O'Neill, and E. Smyth. 2005. A case of multiple cerebral abscesses due to infection with Cladophialophora bantiana. J. Infect. 51:e285-288.

637. Rodriguez, M. M., E. Calvo, C. Serena, M. Marine, F. J. Pastor, and J. Guarro. 2009. Effects of double and triple combinations of antifungal drugs in a murine model of disseminated infection by Scedosporium prolificans. Antimicrob. Agents Chemother. 53:2153-2155.

638. Rodriguez-Tudela, J. L., J. Berenguer, J. Guarro, A. S. Kantarcioglu, R. Horre, G. S. de Hoog, and M. Cuenca-Estrella. 2009. Epidemiology and outcome of Scedosporium prolificans infection, a review of 162 cases. Med. Mycol. 47:359-370

639. Roeijmans, H. J., G. S. De Hoog, C. S. Tan, and M. J. Figge. 1997. Molecular taxonomy and GC/MS of metabolites of Scytalidium hyalinum and Nattrassia mangiferae (Hendersonula toruloidea). J. Med. Vet. Mycol. 35: 181-188.

640. Rohwedder, J. J., J. L. Simmons, H. Colfer, and B. Gatmaitan. 1979. Disseminated Curvularia lunata infection in a football player. Arch. Intern. Med. 139:940-941.

641. Romano, C., R. Bilenchi, C. Alessandrini, and C. Miracco. 1999. Case report. Cutaneous phaeohyphomycosis caused by Cladosporium oxysporum. Mycoses 42:111-115. 
642. Romano, C., M. Fimiani, M. Pellegrino, L. Valenti, L. Casini, C. Miracco, and E. Faggi. 1996. Cutaneous phaeohyphomycosis due to Alternaria tenuissima. Mycoses 39:211-215.

643. Romano, C., E. Paccagnini, and E. M. Difonzo. 2001. Onychomycosis caused by Alternaria spp. in Tuscany, Italy from 1985 to 1999 . Mycoses 44:73-76.

644. Romano, C., L. Valenti, C. Miracco, C. Alessandrini, E. Paccagnini, E. Faggi, and E. M. Difonzo. 1997. Two cases of cutaneous phaeohyphomycosis by Alternaria alternata and Alternaria tenuissima. Mycopathologia 137: 65-74.

645. Romano, C., L. Vanzi, D. Massi, and E. M. Difonzo. 2005. Subcutaneous alternariosis. Mycoses 48:408-412.

646. Ronan, S. G., I. Uzoaru, V. Nadimpalli, J. Guitart, and J. R. Manaligod 1993. Primary cutaneous phaeohyphomycosis: report of seven cases. J. Cutan. Pathol. 20:223-228.

647. Roncoroni, A. J., and J. Smayevsky. 1988. Arthritis and endocarditis from Exophiala jeanselmei infection. Ann. Intern. Med. 108:773.

648. Roosje, P. J., G. S. de Hoog, J. P. Koeman, and T. Willemse. 1993. Phaeohyphomycosis in a cat caused by Alternaria infectoria E. G. Simmons. Mycoses 36:451-454.

649. Rossmann, S. N., P. L. Cernoch, and J. R. Davis. 1996. Dematiaceous fung are an increasing cause of human disease. Clin. Infect. Dis. 22:73-80.

650. Rotowa, N. A., H. J. Shadomy, and S. Shadomy. 1990. In vitro activities of polyene and imidazole antifungal agents against unusual opportunistic fungal pathogens. Mycoses 33:203-211.

651. Rowland, M. D., and W. E. Farrar. 1987. Thorn-induced Phialophora parasitica arthritis treated successfully with synovectomy and ketaconazole. Am. J. Med. Sci. 30:393-395.

652. Ruchel, R., M. Schaffrinski, K. R. Seshan, and G. T. Cole. 2000. Vita staining of fungal elements in deep-seated mycotic lesions during experimental murine mycoses using the parenterally applied optical brightener Blankophor. Med. Mycol. 38:231-237.

653. Ruiz-Diez, B., and J. V. Martinez-Suarez. 2003. Isolation, characterization, and antifungal susceptibility of melanin-deficient mutants of Scedosporium prolificans. Curr. Microbiol. 46:228-232.

654. Rupa, V., M. Jacob, M. S. Mathews, A. Job, M. Kurien, and S. M. Chandi. 2002. Clinicopathological and mycological spectrum of allergic fungal sinusitis in South India. Mycoses 45:364-367.

655. Ryder, N. S., and I. Frank. 1992. Interaction of terbinafine with human serum and serum proteins. J. Med. Vet. Mycol. 30:451-460.

656. Ryoo, N. H., J. S. Ha, D. S. Jeon, J. R. Kim, and E. A. Hwang. 2009 Alternaria peritonitis after contact with a cat. Perit. Dial. Int. 29:235-236.

657. Saberi, H., A. Kashfi, S. Hamidi, S. A. Tabatabai, and P. Mansouri. 2003. Cerebral phaeohyphomycosis masquerading as a parafalcian mass: case report. Surg. Neurol. 60:354-359.

658. Saenz, R. E., W. D. Brown, and C. V. Sanders. 2001. Allergic bronchopulmonary disease caused by Bipolaris hawaiiensis presenting as a necrotizing pneumonia: case report and review of literature. Am. J. Med. Sci. 321:209212.

659. Safdar, A. 2003. Curvularia-favorable response to oral itraconazole therapy in two patients with locally invasive phaeohyphomycosis. Clin. Microbiol. Infect. 9:1219-1223.

660. Saint-Jean, M., G. St-Germain, C. Laferriere, and B. Tapiero. 2007. Hospital-acquired phaeohyphomycosis due to Exserohilum rostratum in a child with leukemia. Can. J. Infect. Dis. Med. Microbiol. 18:200-202.

661. Salama, A. D., T. Rogers, G. M. Lord, R. I. Lechler, and P. D. Mason. 1997. Multiple Cladosporium brain abscesses in a renal transplant patient: aggressive management improves outcome. Transplantation 63:160-162.

662. Salem, F. A., D. W. Kannangara, and R. Nachum. 1983. Cerebral chromomycosis. Arch. Neurol. 40:173-174.

663. Salkin, I. F., J. A. Martinez, and M. E. Kemna. 1986. Opportunistic infection of the spleen caused by Aureobasidium pullulans. J. Clin. Microbiol. 23:828-831.

664. Salkin, I. F., M. R. McGinnis, M. J. Dykstra, and M. G. Rinaldi, 1988 Scedosporium inflatum, an emerging pathogen. J. Clin. Microbiol. 26:498503.

665. Sandhyamani, S., R. Bhatia, L. N. Mohapatra, and S. Roy. 1981. Cerebral cladosporiosis. Surg. Neurol. 15:431-434.

666. Santos, A. L., V. F. Palmeira, S. Rozental, L. F. Kneipp, L. Nimrichter, D. S Alviano, M. L. Rodrigues, and C. S. Alviano. 2007. Biology and pathogenesis of Fonsecaea pedrosoi, the major etiologic agent of chromoblastomycosis. FEMS Microbiol. Rev. 31:570-591.

667. Santosh, V., N. Khanna, S. K. Shankar, L. Pal, S. Das, A. Chandramukhi, and V. R. Kolluri. 1995. Primary mycotic abscess of the brain caused by Fonsecaea pedrosoi. Case report. J. Neurosurg. 82:128-130.

668. Sasama, J., D. A. Sherris, S. H. Shin, G. M. Kephart, E. B. Kern, and J. U. Ponikau. 2005. New paradigm for the roles of fungi and eosinophils in chronic rhinosinusitis. Curr. Opin. Otolaryngol. Head Neck Surg. 13:2-8.

669. Sautter, R. E., M. D. Bliss, D. Morrow, and R. E. Lee. 1984. Isolation of Exophiala jeanselmei associated with esophageal pathology-three cases, laboratory and clinical features. Mycopathologia 87:105-109.

670. Schnadig, V. J., and G. L. Woods. 2009. Histology of fungal infections, p.
79-108. In E. J. Anaissie, M. R. McGinnis, and M. A. Pfaller (ed.), Clinical mycology, 2nd ed. Churchill Livingstone, Philadelphia, PA.

671. Schnitzler, N., H. Peltroche-Llacsahuanga, N. Bestier, J. Zundorf, R. Lutticken, and G. Haase. 1999. Effect of melanin and carotenoids of Exophiala (Wangiella) dermatitidis on phagocytosis, oxidative burst, and killing by human neutrophils. Infect. Immun. 67:94-101.

672. Schonheyder, H. C., H. E. Jensen, W. Gams, O. Nyvad, P. Van Nga, B. Aalbaek, and J. Stenderup. 1996. Late bioprosthetic valve endocarditis caused by Phialemonium aff. curvatum and Streptococcus sanguis: a case report. J. Med. Vet. Mycol. 34:209-214.

673. Schubert, M. S. 2004. Allergic fungal sinusitis. Otolaryngol. Clin. N. Am. 37:301-326.

674. Schubert, M. S. 2004. Allergic fungal sinusitis: pathogenesis and management strategies. Drugs 64:363-374.

675. Schubert, M. S. 2009. Allergic fungal sinusitis: pathophysiology, diagnosis and management. Med. Mycol. 47(Suppl. 1):S324-S330.

676. Schubert, M. S., P. S. Hutcheson, R. J. Graff, L. Santiago, and R. G. Slavin. 2004. HLA-DQB $1 * 03$ in allergic fungal sinusitis and other chronic hypertrophic rhinosinusitis disorders. J. Allergy Clin. Immunol. 114:1376-1383.

677. Scott, I. U., V. Cruz-Villegas, H. W. Flynn, Jr., and D. Miller. 2004. Delayed-onset, bleb-associated endophthalmitis caused by Lecythophora mutabilis. Am. J. Ophthalmol. 137:583-585.

678. Seaworth, B. J., K. J. Kwon-Chung, J. D. Hamilton, and J. R. Perfect. 1983. Brain abscess caused by a variety of Cladosporium trichoides. Am. J. Clin. Pathol. 79:747-752.

679. Segner, S., F. Jouret, J. F. Durant, L. Marot, and N. Kanaan. 2009. Cutaneous infection by Alternaria infectoria in a renal transplant patient. Transpl. Infect. Dis. 11:330-332.

680. Seiberling, K., and P. J. Wormald. 2009. The role of itraconazole in recalcitrant fungal sinusitis. Am. J. Rhinol. Allergy 23:303-306.

681. Sekhon, A. S., J. Galbraith, B. W. Mielke, A. K. Garg, and G. Sheehan. 1992. Cerebral phaeohyphomycosis caused by Xylohypha bantiana, with review of the literature. Eur. J. Epidemiol. 8:387-390.

682. Severo, L. C., M. C. Bassanesi, and A. T. Londero. 1994. Tinea nigra: report of four cases observed in Rio Grande do Sul (Brazil) and a review of Brazilian literature. Mycopathologia 126:157-162.

683. Severo, L. C., F. M. Oliveira, G. Vettorato, and A. T. Londero. 1999 Mycetoma caused by Exophiala jeanselmei. Report of a case successfully treated with itraconazole and review of the literature. Rev. Iberoam. Micol. 16:57-59.

684. Sevigny, G. M., and F. A. Ramos-Caro. 2000. Treatment of chromoblastomycosis due to Fonsecaea pedrosoi with low-dose terbinafine. Cutis 66: $45-46$.

685. Shah, C. V., D. B. Jones, and E. R. Holz. 2001. Microsphaeropsis olivacea keratitis and consecutive endophthalmitis. Am. J. Ophthalmol. 131:142143.

686. Sheikh, S. S., and S. S. Amr. 2007. Mycotic cysts: report of 21 cases including eight pheomycotic cysts from Saudi Arabia. Int. J. Dermatol. 46:388-392.

687. Shelton, B. G., K. H. Kirkland, W. D. Flanders, and G. K. Morris. 2002 Profiles of airborne fungi in buildings and outdoor environments in the United States. Appl. Environ. Microbiol. 68:1743-1753.

688. Shigemori, M., K. Kawakami, T. Kitahara, O. Ijichi, M. Mizota, N. Ikarimoto, and K. Miyata. 1996. Hepatosplenic abscess caused by Curvularia boedijn in a patient with acute monocytic leukemia. Pediatr. Infect. Dis. J. 15:1128-1129.

689. Shigemura, T., K. Agematsu, T. Yamazaki, K. Eriko, G. Yasuda, K. Nishimura, and K. Koike. 2009. Femoral osteomyelitis due to Cladophialophora arxii in a patient with chronic granulomatous disease. Infection 37:469-473.

690. Shin, J. H., S. K. Lee, S. P. Suh, D. W. Ryang, N. H. Kim, M. G. Rinaldi, and D. A. Sutton. 1998. Fatal Hormonema dematioides peritonitis in a patient on continuous ambulatory peritoneal dialysis: criteria for organism identification and review of other known fungal etiologic agents. J. Clin. Microbiol. 36:2157-2163.

691. Shukla, P. K., Z. A. Khan, B. Lal, P. K. Agrawal, and O. P. Srivastava. 1983. Clinical and experimental keratitis caused by the Colletotrichum state of Glomerella cingulata and Acrophialophora fusispora. Sabouraudia 21:137147.

692. Sides, E. H., III, J. D. Benson, and A. A. Padhye. 1991. Phaeohyphomycotic brain abscess due to Ochroconis gallopavum in a patient with malignant lymphoma of a large cell type. J. Med. Vet. Mycol. 29:317-322.

693. Sidrim, J. J. C., R. H. O. Menezes, G. C. Paixao, M. F. G. Rocha, R. S. N. Brilhante, A. M. A. Oliveria, and M. J. N. Diogenes. 1999. Rhinocladiella aquaspersa: limite imprecise entre chromoblastomycose et phaeohyphomycose? J. Mycol. Méd. 9:114-118.

694. Sigler, L., R. C. Summerbell, L. Poole, M. Wieden, D. A. Sutton, M. G. Rinaldi, M. Aguirre, G. W. Estes, and J. N. Galgiani. 1997. Invasive Nattrassia mangiferae infections: case report, literature review, and therapeutic and taxonomic appraisal. J. Clin. Microbiol. 35:433-440.

695. Silva, J. P., W. de Souza, and S. Rozental. 1998. Chromoblastomycosis: a retrospective study of 325 cases on Amazonic Region (Brazil). Mycopathologia 143:171-175. 
696. Silveira, E. R., M. A. Resende, V. S. Mariano, W. A. Coura, L. D. Alkmim, L. B. Vianna, C. E. Starling, G. G. Cruz, L. H. Benicio, A. M. Paula, J. A Gomes, G. D. Santos, M. A. Macedo, R. E. Salum, M. Gontijo, A. L. Rabello, and R. B. Caligiorne. 2003. Brain abscess caused by Cladophialophora (Xylohypha) bantiana in a renal transplant patient. Transpl. Infect. Dis. 5:104-107.

697. Simarro, E., F. Marin, A. Morales, E. Sanz, J. Perez, and J. Ruiz. 2001 Fungemia due to Scedosporium prolificans: a description of two cases with fatal outcome. Clin. Microbiol. Infect. 7:645-647.

698. Simitsopoulou, M., C. Gil-Lamaignere, N. Avramidis, A. Maloukou, S. Lekkas, E. Havlova, L. Kourounaki, D. Loebenberg, and E. Roilides. 2004. Antifungal activities of posaconazole and granulocyte-macrophage colonystimulating factor ex vivo and in mice with disseminated infection due to Scedosporium prolificans. Antimicrob. Agents Chemother. 48:3801-3805.

699. Singal, A., D. Pandhi, S. N. Bhattacharya, S. Das, S. Aggarwal, and K. Mishra. 2008. Pheohyphomycosis caused by Exophiala spinifera: a rare occurrence. Int. J. Dermatol. 47:44-47.

700. Singh, H., S. Irwin, S. Falowski, M. Rosen, L. Kenyon, D. Jungkind, and J. Evans. 2008. Curvularia fungi presenting as a large cranial base meningioma: case report. Neurosurgery 63:e177.

701. Singh, N., R. Agarwal, D. Gupta, M. R. Shivaprakash, and A. Chakrabarti. 2006. An unusual case of mediastinal mass due to Fonsecaea pedrosoi. Eur. Respir. J. 28:662-664.

702. Singh, N., F. Y. Chang, T. Gayowski, and I. R. Marino. 1997. Infections due to dematiaceous fungi in organ transplant recipients: case report and review. Clin. Infect. Dis. 24:369-374.

703. Singh, S. M., J. Naidu, and M. Pouranik. 1990. Ungual and cutaneous phaeohyphomycosis caused by Alternaria alternata and Alternaria chlamydospora. J. Med. Vet. Mycol. 28:275-278.

704. Siu, K., and A. K. Izumi. 2004. Phaeohyphomycosis caused by Coniothyrium. Cutis 73:127-130.

705. Slomovic, A. R., R. K. Forster, and H. Gelender. 1985. Lasiodiplodia theobromae panophthalmitis. Can. J. Ophthalmol. 20:225-228.

706. Smith, J., and D. Andes. 2008. Therapeutic drug monitoring of antifungals: pharmacokinetic and pharmacodynamic considerations. Ther. Drug Monit. 30:167-172.

707. Smith, W. J., R. H. Drew, and J. R. Perfect. 2009. Posaconazole's impact on prophylaxis and treatment of invasive fungal infections: an update. Expert Rev. Anti Infect. Ther. 7:165-181.

708. Sole, M., J. Cano, J. L. Rodriguez-Tudela, J. Ponton, D. A. Sutton, R. Perrie, J. Gene, V. Rodriguez, and J. Guarro. 2003. Molecular typing of clinical and environmental isolates of Scedosporium prolificans by intersimple-sequence-repeat polymerase chain reaction. Med. Mycol. 41:293300.

709. Song, M. J., J. H. Lee, and N. Y. Lee. 2009. Fatal Scedosporium prolificans infection in a paediatric patient with acute lymphoblastic leukaemia. Mycoses [Epub ahead of print.] doi:10.1111/j.1439-0507.2009.01765.x.

710. Sood, N., H. C. Gugnani, J. Guarro, A. Paliwal-Joshi, and V. K. Vijayan. 2007. Subcutaneous phaeohyphomycosis caused by Alternaria alternata in an immunocompetent patient. Int. J. Dermatol. 46:412-413.

711. Sood, P., V. Dogra, A. Thakur, B. Mishra, A. Mandal, and S. Sinha. 2000 Brain abscess due to Xylohypha bantiana. Scand. J. Infect. Dis. 32:708-709.

712. Sparrow, S. A., L. A. Hallam, B. E. Wild, and D. L. Baker. 1992. Scedosporium inflatum: first case report of disseminated infection and review of the literature. Pediatr. Hematol. Oncol. 9:293-295.

713. Spielberger, R. T., B. R. Tegtmeier, M. R. O'Donnell, and J. I. Ito. 1995. Fatal Scedosporium prolificans (S. inflatum) fungemia following allogeneic bone marrow transplantation: report of a case in the United States. Clin. Infect. Dis. 21:1067.

714. Srinivasan, A., B. L. Wickes, A. M. Romanelli, L. Debelenko, J. E. Rubnitz, D. A. Sutton, E. H. Thompson, A. W. Fothergill, M. G. Rinaldi, R. T. Hayden, and J. L. Shenep. 2009. Cutaneous infection caused by Macrophomina phaseolina in a child with acute myeloid leukemia. J. Clin. Microbiol. 47:1969-1972.

715. Srinivasan, M. 2004. Fungal keratitis. Curr. Opin. Ophthalmol. 15:321-327.

716. Steinbach, W. J., W. A. Schell, J. L. Miller, and J. R. Perfect. 2003. Scedosporium prolificans osteomyelitis in an immunocompetent child treated with voriconazole and caspofungin, as well as locally applied polyhexamethylene biguanide. J. Clin. Microbiol. 41:3981-3985.

717. Strahilevitz, J., G. Rahav, H. J. Schroers, R. C. Summerbell, Z. Amitai, A Goldschmied-Reouven, E. Rubinstein, Y. Schwammenthal, M. S. Feinberg, Y. Siegman-Igra, E. Bash, I. Polacheck, A. Zelazny, S. J. Howard, P. Cibotaro, O. Shovman, and N. Keller. 2005. An outbreak of Phialemonium infective endocarditis linked to intracavernous penile injections for the treatment of impotence. Clin. Infect. Dis. 40:781-786.

718. Studahl, M., T. Backteman, F. Stalhammar, E. Chryssanthou, and B. Petrini. 2003. Bone and joint infection after traumatic implantation of Scedosporium prolificans treated with voriconazole and surgery. Acta Paediatr. 92:980-982.

719. Sudduth, E. J., A. J. Crumbley III, and W. E. Farrar. 1992. Phaeohyphomycosis due to Exophiala species: clinical spectrum of disease in humans. Clin. Infect. Dis. 15:639-644.
720. Summerbell, R. C., S. Krajden, R. Levine, and M. Fuksa. 2004. Subcutaneous phaeohyphomycosis caused by Lasiodiplodia theobromae and successfully treated surgically. Med. Mycol. 42:543-547.

721. Surash, S., A. Tyagi, G. S. De Hoog, J. S. Zeng, R. C. Barton, and R. P. Hobson. 2005. Cerebral phaeohyphomycosis caused by Fonsecaea monophora. Med. Mycol. 43:465-472.

722. Sutton, D. A. 2008. Basic mycology, p. 15-35. In D. R. Hospenthal and M. G. Rinaldi (ed.), Diagnosis and treatment of human mycoses. Humana Press, Towata, NJ.

723. Sutton, D. A. 2007. Specimen collection, transport, and processing: mycology, p. 1728-1735. In P. R. Murray, E. J. Baron, J. H. Jorgensen, M. L. Landry, and M. A. Pfaller (ed.), Manual of clinical microbiology, 9th ed. ASM Press, Washington, DC

724. Sutton, D. A., M. G. Rinaldi, S. E. Sanche. 2009. Dematiaceous fungi, p. 329-354. In E. J. Anaissie, M. R. McGinnis, and M. A. Pfaller (ed.). Clinica mycology, 2nd ed. Elsevier, Philadelphia, PA

725. Sutton, D. A., M. G. Rinaldi, and M. Kielhofner. 2004. First U.S. report of subcutaneous phaeohyphomycosis caused by Veronaea botryosa in a heart transplant recipient and review of the literature. J. Clin. Microbiol. 42: 2843-2846.

726. Sutton, D. A., M. Slifkin, R. Yakulis, and M. G. Rinaldi. 1998. U.S. case report of cerebral phaeohyphomycosis caused by Ramichloridium obovoideum (R. mackenziei): criteria for identification, therapy, and review of other known dematiaceous neurotropic taxa. J. Clin. Microbiol. 36:708-715.

727. Sutton, D. A., W. D. Timm, G. Morgan-Jones, and M. G. Rinaldi. 1999 Human phaeohyphomycotic osteomyelitis caused by the coelomycete Phomopsis Saccardo 1905: criteria for identification, case history, and therapy. J. Clin. Microbiol. 37:807-811.

728. Suzuki, Y., S. Udagawa, H. Wakita, N. Yamada, H. Ichikawa, F. Furukawa, and M. Takigawa. 1998. Subcutaneous phaeohyphomycosis caused by Geniculosporium species; a new fungal pathogen. Br. J. Dermatol. 138:346350 .

729. Symmers, W. S. C. 1960. A case of cerebral chromoblastomycosis (Cladosporiosis) occurring in Britain as a complication of polyarteritis treated with cortisone. Brain 83:37-51.

730. Szaniszlo, P. J. 2002. Molecular genetic studies of the model dematiaceous pathogen Wangiella dermatitidis. Int. J. Med. Microbiol. 292:381-390.

731. Taj-Aldeen, S. J., M. Almaslamani, A. Alkhal, I. A. Bozom, A. M. Romanelli, B. L. Wickes, A. W. Fothergill, and D. A. Sutton. 2010. Cerebral phaeohyphomycosis due to Rhinocladiella mackenziei (formerly Ramichloridium mackenziei): a taxonomic update and review of the literature. Med. Mycol. 48:546-556.

732. Taj-Aldeen, S. J., A. A. Hilal, and W. A. Schell. 2004. Allergic fungal rhinosinusitis: a report of 8 cases. Am. J. Otolaryngol. 25:213-218.

733. Takei, H., J. C. Goodman, and S. Z. Powell. 2007. Cerebral phaeohyphomycosis caused by Cladophialophora bantiana and Fonsecaea monophora: report of three cases. Clin. Neuropathol. 26:21-27.

734. Tamm, M., M. Malouf, and A. Glanville. 2001. Pulmonary Scedosporium infection following lung transplantation. Transplant. Infect. Dis. 3:189-194.

735. Tan, D. H., L. Sigler, C. F. Gibas, and I. W. Fong. 2008. Disseminated fungal infection in a renal transplant recipient involving Macrophomina phaseolina and Scytalidium dimidiatum: case report and review of taxonomic changes among medically important members of the Botryosphaeriaceae. Med. Mycol. 46:285-292.

736. Tan, H. P., H. E. Wahlstrom, J. U. Zamora, and T. Hassanein. 1997. Aureobasidium pneumonia in a post liver transplant recipient: a case report. Hepatol. Gastroenterol. 44:1215-1218.

737. Taylor, J. W., D. J. Jacobson, S. Kroken, T. Kasuga, D. M. Geiser, D. S. Hibbett, and M. C. Fisher. 2000. Phylogenetic species recognition and species concepts in fungi. Fungal Genet. Biol. 31:21-32.

738. Tekkok, I. H., M. J. Higgins, and E. C. Ventureyra. 1996. Posttraumatic gas-containing brain abscess caused by Clostridium perfringens with unique simultaneous fungal suppuration by Myceliophthora thermophila: case report. Neurosurgery 39:1247-1251.

739. Terra, F., T. M. Fonseca, and O. E. Area Leao. 1922. Novo typo de dermatite verrucosa mycose por Achroteca com associacao de leishmaniose. Brazil Med. 36:363-368.

740. Terreni, A. A., A. F. DiSalvo, A. S. Baker, Jr., W. B. Crymes, P. R. Morris, and H. Dowda, Jr. 1990. Disseminated Dactylaria gallopava infection in a diabetic patient with chronic lymphocytic leukemia of the T-cell type. Am. J. Clin. Pathol. 94:104-107.

741. Tessari, G., A. Forni, R. Ferretto, M. Solbiati, G. Faggian, A. Mazzucco, and A. Barba. 2003. Lethal systemic dissemination from a cutaneous infection due to Curvularia lunata in a heart transplant recipient. J. Eur. Acad. Dermatol. Venereol. 17:440-442.

742. Thomas, C., D. Mileusnic, R. B. Carey, M. Kampert, and D. Anderson. 1999. Fatal Chaetomium cerebritis in a bone marrow transplant patient. Hum. Pathol. 30:874-879.

743. Thomas, P. A. 2003. Current perspectives on ophthalmic mycoses. Clin. Microbiol. Rev. 16:730-797.

744. Thomas, P. A. 2003. Fungal infections of the cornea. Eye 17:852-862. 
745. Thompson, G. R., and J. S. Lewis. 2010. Pharmacology and clinical use of voriconazole. Expert Opin. Drug Metab. Toxicol. 6:83-94.

746. Tintelnot, K., G. S. De Hoog, E. Thomas, W. I. Steudel, K. Huebner, and H. P. Seeliger. 1991. Cerebral phaeohyphomycosis caused by an Exophiala species. Mycoses 34:239-244.

747. Tintelnot, K., G. Just-Nubling, R. Horre, B. Graf, I. Sobottka, M. Seibold, A. Haas, U. Kaben, and G. S. De Hoog. 2009. A review of German Scedosporium prolificans cases from 1993 to 2007. Med. Mycol. 47:351-358.

748. Tintelnot, K., P. von Hunnius, G. S. de Hoog, A. Polak-Wyss, E. Gueho, and F. Masclaux. 1995. Systemic mycosis caused by a new Cladophialophora species. J. Med. Vet. Mycol. 33:349-354.

749. Tokuhisa, Y., Y. Hagiya, M. Hiruma, and K. Nishimura. 2010. Phaeohyphomycosis of the face caused by Exophiala oligosperma. Mycoses [Epub ahead of pring.] doi:10.1111/j.1439-0507.2009.01845.x.

750. Tong, S. Y., A. Y. Peleg, J. Yoong, R. Handke, J. Szer, and M. Slavin. 2007 Breakthrough Scedosporium prolificans infection while receiving voriconazole prophylaxis in an allogeneic stem cell transplant recipient. Transpl. Infect. Dis. 9:241-243.

751. Torres-Rodriguez, J. M., M. P. Gonzalez, J. M. Corominas, and R. M Pujol. 2005. Successful thermotherapy for a subcutaneous infection due to Alternaria alternata in a renal transplant recipient. Arch. Dermatol. 141: 1171-1173.

752. Tosti, A., B. M. Piraccini, S. Lorenzi, and M. Iorizzo. 2003. Treatment of non-dermatophyte mold and Candida onychomycosis. Dermatol. Clin. 21: 491-497.

753. Travis, W. D., K. J. Kwon-Chung, D. E. Kleiner, A. Geber, W. Lawson, H. I Pass, and D. Henderson. 1991. Unusual aspects of allergic bronchopulmonary fungal disease: report of two cases due to Curvularia organisms associated with allergic fungal sinusitis. Hum. Pathol. 22:1240-1248.

754. Trinh, J. V., W. J. Steinbach, W. A. Schell, J. Kurtzberg, S. S. Giles, and J. R. Perfect. 2003. Cerebral phaeohyphomycosis in an immunodeficient child treated medically with combination antifungal therapy. Med. Mycol. 41:339-345.

755. Tsai, C. Y., Y. C. Lu, L. Wang, T. L. Hsu, and J. Sung. 1966. Systemic chromoblastomycosis due to Hormodendrum dermatitidis (Kano) Conant. Am. J. Clin. Pathol. 46:103-114.

756. Tsai, H. F., Y. C. Chang, R. G. Washburn, M. H. Wheeler, and K. J. Kwon-Chung. 1998. The developmentally regulated alb1 gene of Aspergillus fumigatus: its role in modulation of conidial morphology and virulence. J. Bacteriol. 180:3031-3038.

757. Tsai, H. F., M. H. Wheeler, Y. C. Chang, and K. J. Kwon-Chung. 1999. A developmentally regulated gene cluster involved in conidial pigment biosynthesis in Aspergillus fumigatus. J. Bacteriol. 181:6469-6477.

758. Tu, E. Y. 2009. Alternaria keratitis: clinical presentation and resolution with topical fluconazole or intrastromal voriconazole and topical caspofungin. Cornea 28:116-119.

759. Tunuguntla, A., M. M. Saad, J. Abdalla, and J. W. Myers. 2005. Multiple brain abscesses caused by Cladophialophora bantianum: a challenging case. Tenn. Med. 98:227-. 228:235.

760. Turiansky, G. W., P. M. Benson, L. C. Sperling, P. Sau, I. F. Salkin, M. R. McGinnis, and W. D. James. 1995. Phialophora verrucosa: a new cause of mycetoma. J. Am. Acad. Dermatol. 32:311-315.

761. Uberti-Foppa, C., L. Fumagalli, N. Gianotti, A. M. Viviani, R. Vaiani, and E. Gieho. 1995. First case of osteomyelitis due to Phialophora richardsiae in a patient with HIV infection. AIDS 9:975-976.

762. Ujhelyi, M. R., R. H. Raasch, C. M. van der Horst, and W. D. Mattern. 1990. Treatment of peritonitis due to Curvularia and Trichosporon with amphotericin B. Rev. Infect. Dis. 12:621-627.

763. Umabala, P., V. Lakshmi, A. R. Murthy, V. S. Prasad, C. Sundaram, and H Beguin. 2001. Isolation of a Nodulisporium species from a case of cerebral phaeohyphomycosis. J. Clin. Microbiol. 39:4213-4218.

764. Umemoto, N., T. Demitsu, M. Kakurai, K. Sasaki, R. Azuma, E. Iida, K Yoneda, M. Kawasaki, and T. Mochizuki. 2009. Two cases of cutaneous phaeohyphomycosis due to Exophiala jeanselmei: diagnostic significance of direct microscopical examination of the purulent discharge. Clin. Exp. Dermatol. 34:e351-353.

765. Vachharajani, T. J., F. Zaman, S. Latif, R. Penn, and K. D. Abreo. 2005. Curvularia geniculata fungal peritonitis: a case report with review of literature. Int. Urol. Nephrol. 37:781-784.

766. van de Sande, W. W., J. de Kat, J. Coppens, A. O. Ahmed, A. Fahal, H. Verbrugh, and A. van Belkum. 2007. Melanin biosynthesis in Madurella mycetomatis and its effect on susceptibility to itraconazole and ketoconazole. Microbes Infect. 9:1114-1123.

767. van de Sande, W. W., A. Luijendijk, A. O. Ahmed, I. A. Bakker-Woudenberg, and A. van Belkum. 2005. Testing of the in vitro susceptibilities of Madurella mycetomatis to six antifungal agents by using the Sensititre system in comparison with a viability-based 2,3-bis(2-methoxy-4-nitro-5-sulfophenyl)-5-[(phenylamino)carbonyl]-2H-tetrazolium hydroxide (XTT) assay and a modified NCCLS method. Antimicrob. Agents Chemother. 49:13641368 .

768. van Duin, D., A. Casadevall, and J. D. Nosanchuk. 2002. Melanization of Cryptococcus neoformans and Histoplasma capsulatum reduces their suscep- tibilities to amphotericin B and caspofungin. Antimicrob. Agents Chemother. 46:3394-3400.

769. Vartian, C. V., D. M. Shlaes, A. A. Padhye, and L. Ajello. 1985. Wangiella dermatitidis endocarditis in an intravenous drug user. Am. J. Med. 78:703707.

770. Velazquez, L. F., A. Restrepo, and G. Calle. 1976. Cromomicosis: experiencia de doce azos. Acta Med. Colomb. 1:165-171.

771. Venarske, D. L., and R. D. deShazo. 2002. Sinobronchial allergic mycosis: the SAM syndrome. Chest 121:1670-1676.

772. Ventin, M., C. Ramirez, and J. Garau. 1987. Exophiala dermatitidis de Hoog from a valvular aortal prothesis. Mycopathologia 99:45-46.

773. Verkley, G. J. M., M. da Silva, D. T. Wicklow, and P. W. Crous. 2004 Paraconiothyrium, a new genus to accommodate the mycoparasite Coniothyrium minitans, anamorphs of Paraphaeosphaeria, and four new species. Stud. Mycol. 50:323-335.

774. Vermes, A., H. J. Guchelaar, and J. Dankert. 2000. Flucytosine: a review of its pharmacology, clinical indications, pharmacokinetics, toxicity and drug interactions. J. Antimicrob. Chemother. 46:171-179.

775. Vidal, M. S., L. G. de Castro, S. C. Cavalecate, and S. Lacaz Cda. 2003. Immunoprecipitation techniques and Elisa in the detection of anti-Fonsecaea pedrosoi antibodies in chromoblastomycosis. Rev. Inst. Med. Trop. Sao Paulo 45:315-318.

776. Vieira, M. R., A. Milheiro, and F. A. Pacheco. 2001. Phaeohyphomycosis due to Cladosporium cladosporioides. Med. Mycol. 39:135-137.

777. Vijaykrishna, D., L. Mostert, R. Jeewon, W. Gams, K. D. Hyde, and P. W. Crous. 2005. Pleurostomophora, an anamorph of Pleurostoma (Calosphaeriales), a new anamorph genus morphologically similar to Phialophora. Stud. Mycol. 50:387-395.

778. Villalba, E., and J. F. Yegres. 1988. Detection of circulating antibodies in patients affected by chromoblastomycosis by Cladosporium carrionii using double immunodiffusion. Mycopathologia 102:17-19.

779. Vincente, V. A., D. Attili-Angelis, M. R. Pie, F. Queiroz-Telles, L. M. Cruz M. J. Najafzadeh, G. S. de Hoog, J. Zhao, and A. Pizzirani-Kleiner. 2008. Environmental isolation of black yeast-like fungi involved in human infection. Stud. Mycol. 61:137-144.

780. Vitale, R. G., and G. S. De Hoog. 2002. Molecular diversity, new species and antifungal susceptibilities in the Exophiala spinifera clade. Med. Mycol. 40:545-556.

781. Vitale, R. G., G. S. De Hoog, and P. E. Verweij. 2003. In vitro activity of amphotericin B, itraconazole, terbinafine and 5-fluocytosine against Ex ophiala spinifera and evaluation of post-antifungal effects. Med. Mycol. 41:301-307.

782. Vitale, R. G., M. Perez-Blanco, and G. S. De Hoog. 2009. In vitro activity of antifungal drugs against Cladophialophora species associated with human chromoblastomycosis. Med. Mycol. 47:35-40.

783. Vlassopoulos, D., G. Kouppari, D. Arvanitis, K. Papaefstathiou, A. Dounavis, A. Velegraki, and V. Hadjiconstantinou. 2001. Wangiella dermatitidis peritonitis in a CAPD patient. Perit. Dial. Int. 21:96-97.

784. Vogelgesang, S. A., J. W. Lockard, M. J. Quinn, and J. A. Hasbargen. 1990 Alternaria peritonitis in a patient undergoing continuous ambulatory peritoneal dialysis. Perit. Dial. Int. 10:313.

785. Vollmer, T., M. Stormer, K. Kleesiek, and J. Dreier. 2008. Evaluation of novel broad-range real-time PCR assay for rapid detection of human pathogenic fungi in various clinical specimens. J. Clin. Microbiol. 46:1919-1926.

786. Vukmir, R. B., S. Kusne, P. Linden, W. Pasculle, A. W. Fothergill, J. Sheaffer, J. Nieto, R. Segal, H. Merhav, and A. J. Martinez. 1994. Successful therapy for cerebral phaeohyphomycosis due to Dactylaria gallopava in a liver transplant recipient. Clin. Infect. Dis. 19:714-719.

787. Walz, R., M. Bianchin, M. L. Chaves, M. R. Cerski, L. C. Severo, and A. T. Londero. 1997. Cerebral phaeohyphomycosis caused by Cladophialophora bantiana in a Brazilian drug abuser. J. Med. Vet. Mycol. 35:427-431.

788. Wang, T. K., W. Chiu, S. Chim, T. M. Chan, S. S. Wong, and P. L. Ho. 2003 Disseminated Ochroconis gallopavum infection in a renal transplant recipient: the first reported case and a review of the literature. Clin. Nephrol. 60:415-423.

789. Warnock, D. W. 2007. Taxonomy and classification of fungi, p. 17211727. In P. R. Murray, E. J. Baron, J. H. Jorgensen, M. L. Landry, and M. A. Pfaller (ed.), Manual of clinical microbiology, 9th ed. ASM Press, Washington, DC.

790. Watson, K. C. 1962. Cerebral chromoblastomycosis. J. Pathol. Bacteriol. 84:233-237.

791. Watson, K. C., and G. M. Lines. 1957. Brain abscess due to the fungus Hormodendrum. S. African Med. J. 31:1081-1082.

792. Weber, E., C. Görke, and D. Begerow. 2002. The Lecythophora-Coniochaeta complelx: II. Molecular studies based on sequences of the large subunit of ribosomal DNA. Nova Hedwigia 74:187-200.

793. Weinberger, M., I. Mahrshak, N. Keller, A. Goldscmied-Reuven, N. Amariglio, M. Kramer, A. Tobar, Z. Samra, S. D. Pitlik, M. G. Rinaldi, E. Thompson, and D. Sutton. 2006. Isolated endogenous endophthalmitis due to a sporodochial-forming Phialemonium curvatum acquired through intracavernous autoinjections. Med. Mycol. 44:253-259.

794. Westerman, D. A., B. R. Speed, and H. M. Prince. 1999. Fatal disseminated 
infection by Scedosporium prolificans during induction therapy for acute leukemia: a case report and literature review. Pathology 31:393-394.

795. Wheeler, M. H., and A. A. Bell. 1988. Melanins and their importance in pathogenic fungi. Curr. Top. Med. Mycol. 2:338-387.

796. Whyte, M., H. Irving, P. O'Regan, M. Nissen, D. Siebert, and R. Labrom. 2005. Disseminated Scedosporium prolificans infection and survival of a child with acute lymphoblastic leukemia. Pediatr. Infect. Dis. J. 24:375-377.

797. Widmer, F., L. C. Wright, D. Obando, R. Handke, R. Ganendren, D. H. Ellis, and T. C. Sorrell. 2006. Hexadecylphosphocholine (miltefosine) has broad-spectrum fungicidal activity and is efficacious in a mouse model of cryptococcosis. Antimicrob. Agents Chemother. 50:414-421.

798. Wiest, P. M., K. Wiese, M. R. Jacobs, A. B. Morrissey, T. I. Abelson, W. Witt, and M. M. Lederman. 1987. Alternaria infection in a patient with acquired immunodeficiency syndrome: case report and review of invasive alternaria infections. Rev. Infect. Dis. 9:799-803.

799. Wilhelmus, K. R. 2005. Climatology of dematiaceous fungal keratitis. Am. J. Ophthalmol. 140:1156-1157.

800. Wilhelmus, K. R., and D. B. Jones. 2001. Curvularia keratitis. Trans. Am Ophthalmol. Soc. 99:111-130.

801. Willinger, B., G. Kopetzky, F. Harm, P. Apfalter, A. Makristathis, A. Berer A. Bankier, and S. Winkler. 2004. Disseminated infection with Nattrassia mangiferae in an immunosuppressed patient. J. Clin. Microbiol. 42:478-480.

802. Wilson, C. M., E. J. O'Rourke, M. R. McGinnis, and I. F. Salkin. 1990 Scedosporium inflatum: clinical spectrum of a newly recognized pathogen. J. Infect. Dis. 161:102-107.

803. Wilson, E. 1982. Cerebral abscess caused by Cladosporium bantianum. Case report. Pathology 14:91-96.

804. Wise, K. A., B. R. Speed, D. H. Ellis, and J. H. Andrew. 1993. Two fatal infections in immunocompromised patients caused by Scedosporium inflatum. Pathology 25:187-189.

805. Woo, P. C., S. K. Lau, A. H. Ngan, H. Tse, E. T. Tung, and K. Y. Yuen. 2008 Lasiodiplodia theobromae pneumonia in a liver transplant recipient. J. Clin. Microbiol. 46:380-384.

806. Wood, G. M., J. G. McCormack, D. B. Muir, D. H. Ellis, M. F. Ridley, R. Pritchard, and M. Harrison. 1992. Clinical features of human infection with Scedosporium inflatum. Clin. Infect. Dis. 14:1027-1033.

807. Woollons, A., C. R. Darley, S. Pandian, P. Arnstein, J. Blackee, and J. Paul. 1996. Phaeohyphomycosis caused by Exophiala dermatitidis following intraarticular steroid injection. Br. J. Dermatol. 135:475-477.

808. Xi, L., C. Lu, J. Sun, X. Li, H. Liu, J. Zhang, Z. Xie, and G. S. De Hoog 2009. Chromoblastomycosis caused by a meristematic mutant of Fonsecaea monophora. Med. Mycol. 47:77-80.

809. Xi, L., J. Sun, C. Lu, H. Liu, Z. Xie, K. Fukushima, K. Takizawa, M. J. Najafzadeh, and G. S. De Hoog. 2009. Molecular diversity of Fonsecaea (Chaetothyriales) causing chromoblastomycosis in southern China. Med. Mycol. 47:27-33.

810. Xie, Z., J. Zhang, L. Xi, X. Li, L. Wang, C. Lu, and J. Sun. 2010. A chronic chromoblastomycosis model by Fonsecaea monophora in Wistar rat. Med. Mycol. 48:201-206.

811. Yamagishi, Y., K. Kawasaki, and H. Ishizaki. 1997. Mitochondrial DNA analysis of Phialophora verrucosa. Mycoses 40:329-334.

812. Yangco, B. G., D. TeStrake, and J. Okafor. 1984. Phialophora richardsiae isolated from infected human bone: morphological, physiological and antifungal susceptibility studies. Mycopathologia 86:103-111.

813. Yau, Y. C., J. de Nanassy, R. C. Summerbell, A. G. Matlow, and S. E. Richardson. 1994. Fungal sternal wound infection due to Curvularia lunata in a neonate with congenital heart disease: case report and review. Clin. Infect. Dis. 19:735-740.

814. Yeghen, T., L. Fenelon, C. K. Campbell, D. W. Warnock, A. V. Hoffbrand, H. G. Prentice, and C. C. Kibbler. 1996. Chaetomium pneumonia in patient with acute myeloid leukaemia. J. Clin. Pathol. 49:184-186.

815. Yehia, M., M. Thomas, H. Pilmore, W. Van Der Merwe, and I. Dittmer. 2004. Subcutaneous black fungus (phaeohyphomycosis) infection in renal transplant recipients: three cases. Transplantation 77:140-142.

816. Yoshimori, R. N., R. A. Moore, H. H. Itabashi, and D. G. Fujikawa. 1982 Phaeohyphomycosis of brain: granulomatous encephalitis caused by Drechslera spicifera. Am. J. Clin. Pathol. 77:363-370.

817. Young, C. N., J. G. Swart, D. Ackermann, and K. Davidge-Pitts. 1978. Nasal obstruction and bone erosion caused by Drechslera hawaiiensis. J. Laryngol. Otol. 92:137-143.

818. Yu, J., S. Yang, Y. Zhao, and R. Li. 2006. A case of subcutaneous phaeohyphomycosis caused by Chaetomium globosum and the sequences analysis of C. globosum. Med. Mycol. 44:541-545.

819. Yurlova, N. A., and G. S. de Hoog. 2002. Exopolysaccharides and capsules in human pathogenic Exophiala species. Mycoses 45:443-448.

820. Yurlova, N. A., G. S. de Hoog, and A. H. G. Gerrits van den Ende. 1999. Taxonomy of Aureobasidium and allied genera. Stud. Mycol. 43:63-69.

821. Yustes, C., and J. Guarro. 2005. In vitro synergistic interaction between amphotericin B and micafungin against Scedosporium spp. Antimicrob. Agents Chemother. 49:3498-3500.

822. Zaharopoulos, P., V. J. Schnadig, K. D. Davie, R. E. Boudreau, and V. W. Weedn. 1988. Multiseptate bodies in systemic phaeohyphomycosis diagnosed by fine needle aspiration cytology. Acta Cytol. 32:885-891.

823. Zalar, P., G. S. de Hoog, and N. Gunde-Cimerman. 1999. Ecology of halotolerant dothideaceous black yeasts. Stud. Mycol. 43:38-48.

824. Zalar, P., C. Gostincar, G. S. de Hoog, V. Ursic, M. Sudhadham, and N. Gunde-Cimerman. 2008. Redefinition of Aureobasidium pullulans and its varieties. Stud. Mycol. 61:21-38.

825. Zeng, J. S., D. A. Sutton, A. W. Fothergill, M. G. Rinaldi, M. J. Harrak, and G. S. de Hoog. 2007. Spectrum of clinically relevant Exophiala species in the United States. J. Clin. Microbiol. 45:3713-3720.

826. Zeppenfeldt, G., N. Richard-Yegres, F. Yegres, and R. Hernández. 1994 Cladosporium carrionii: hongo dimorfo en cactáceas de la zona endémica para la cromomicosis en Venezuela. Rev. Iberoam. Micol. 11:61-63.
Sanjay Revankar, M.D., is an Associate Professor at Wayne State University, Department of Medicine, Division of Infectious Diseases, Detroit, MI. He completed his residency in internal medicine at the University of Michigan and fellowships in infectious diseases and mycology at the University of Texas Health Science Center, San Antonio, and the Fungus Testing Laboratory, respectively. His research interests include basic and clinical mycology, especially unusual mold infections.

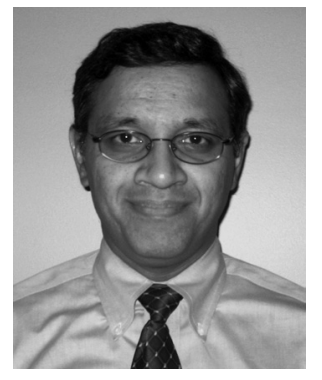

Deanna A. Sutton, Ph.D., MT,SM(ASCP), $\mathrm{RM}, \mathrm{SM}(\mathrm{NRCM})$, is an Associate Professor in the Department of Pathology at the University of Texas Health Science Center in San Antonio, TX, and the Administrative Director of the Fungus Testing Laboratory (FTL). As a member of the FTL, her research interests encompass fungal taxonomy, antifungal susceptibility trends, and identification of rare molds encountered in human and veterinary medicine. She has taught numerous medical mycology courses and has been an invited workshop presenter both nationally and internationally. She currently serves as an Associate Editor of Medical Mycology; is on the editorial board of several peer-reviewed journals, including the Journal of Clinical Microbiology and Antimicrobial Agents and Chemotherapy; and has written extensively on invasive fungal infections caused by uncommon and emerging molds in humans and animals. 